\title{
Characterization of diamond sensors in ATLAS pixel read-out electronics
}

by

\section{Adam Robichaud}

\author{
A Thesis submitted to \\ the Faculty of Graduate and Postdoctoral Affairs \\ in partial fulfilment of \\ the requirements for the degree of \\ Master of Science
}

Ottawa-Carleton Institute for

Physics

Department of Physics
Carleton University
Ottawa, Ontario, Canada

December 2010

Copyright (C)

2010 - Adam Robichaud 
Library and Archives

Canada

Published Heritage

Branch

395 Wellington Street

Ottawa ON K1A ON4

Canada
Bibliothèque et

Archives Canada

Direction du

Patrimoine de l'édition

395 , rue Wellington

Ottawa ON K1A ON4

Canada
Your file Votre reférence

ISBN: 978-0-494-79586-6

Our file Notre référence

ISBN: 978-0-494-79586-6
NOTICE:

The author has granted a nonexclusive license allowing Library and Archives Canada to reproduce, publish, archive, preserve, conserve, communicate to the public by telecommunication or on the Internet, loan, distribute and sell theses worldwide, for commercial or noncommercial purposes, in microform, paper, electronic and/or any other formats.

The author retains copyright ownership and moral rights in this thesis. Neither the thesis nor substantial extracts from it may be printed or otherwise reproduced without the author's permission.
AVIS:

L'auteur a accordé une licence non exclusive permettant à la Bibliothèque et Archives Canada de reproduire, publier, archiver, sauvegarder, conserver, transmettre au public par télécommunication ou par l'Internet, prêter, distribuer et vendre des thèses partout dans le monde, à des fins commerciales ou autres, sur support microforme, papier, électronique et/ou autres formats.

L'auteur conserve la propriété du droit d'auteur et des droits moraux qui protège cette thèse. $\mathrm{Ni}$ la thèse ni des extraits substantiels de celle-ci ne doivent être imprimés ou autrement reproduits sans son autorisation.
In compliance with the Canadian Privacy Act some supporting forms may have been removed from this thesis.

While these forms may be included in the document page count, their removal does not represent any loss of content from the thesis.
Conformément à la loi canadienne sur la protection de la vie privée, quelques formulaires secondaires ont été enlevés de cette thèse.

Bien que ces formulaires aient inclus dans la pagination, il n'y aura aucun contenu manquant.

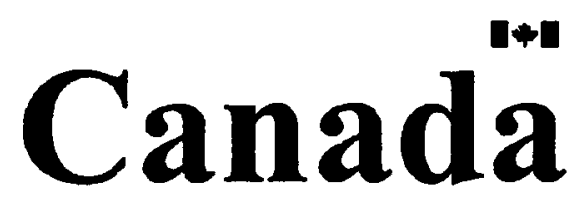




\section{Abstract}

Particle tracking in high energy experiments such as ATLAS require the use of semiconducting pixel detectors. The proposed luminosity upgrade of the LHC will create an environment which will require those pixel detectors to survive fluences up to $10^{16}$ particles per $\mathrm{cm}^{2}$ over the duration of their use. Synthetic diamonds are being researched for viability as pixel detectors in an sLHC environment.

A single crystal diamond detector using ATLAS pixel read-out electronics has been irradiated to $0.7 \times 10^{15}$ protons per $\mathrm{cm}^{2}$, and the charge collection inside a pixel cell was studied in a high energy particle beam. Software has been created to determine data quality, and an analysis package has been developed to perform event selection, hit reconstruction, device alignment, track reconstruction and analyze charge collected for existing beam test data. New USB-based hardware was implemented to assist in the characterization and calibration of ATLAS pixel devices, and the hardware's feasibility for use in a beam telescope has been studied. 
This thesis is dedicated to my lovely wife for her infinite support and understanding during its creation. 


\section{Acknowledgments}

I would like to acknowledge the following people for their support during the course of my studies and beyond:

To my supervisor David Asner who provided me with the opportunity to do this research - that kindness will never be forgotten, and I sincerely hope you found your investment in me was worth the risk. Thanks go out to Alessandro La Rosa and Josh Moss for taking time from their extremely busy schedules to train me on the inner workings of test-beam execution and analysis, and also to Jens Weingarten for solving countless of my hardware issues with the "Jens touch".

I would also like to express my gratitude to Rick Ueno, Tom McCarthy and Miro Vujicic for dragging me through the trenches of graduate studies, and give a special thanks to Kate Whalen for her assistance in making grammatical sense of this mess.

I would like to acknowledge the following people for their support outside of academia: Paras Naik for being an omnipresent force of good in my life. Brian Moziak, Tudor Costin and Josh Turner for their assistance in developing my passions while balancing my eclectic personality, my mother-in-law Sandy Ryan for being extremely supportive and ever concerned about my well being, and Linda Pelletier for going above and beyond the call of duty as a caregiver and friend. You have all been invaluable to my development as a person, and I owe you all a debt of gratitude.

I am eternally indebted to my father Delbe whose support and guidance were vital to my success, my mother Linda who continues to sacrifice for my wellbeing to this 
very day, and to my younger brothers Matthew and Andrew who continue to tolerate my craziness, while providing that "brotherly love" that keeps you going on the right path. I am the person I am today largely due to your influences - feel free to make what you will of that.

I thank my dog Kobi for practicing what must have been endless restraint and not destroying my laptop while I wrote this thesis. I also thank him for his leering gaze at 5am that reminded me sleeping is not optional if one hopes to function.

Finally to my wife, Katelyn: I thank you endlessly for the support and love you have given me over the years. You are the engine that drives me up the mountain of achievement, and you bring order into the chaos that is my life. Without you, I surely would have quit years ago. 


\section{Table of Contents}

$\begin{array}{ll}\text { Abstract } & \text { ii }\end{array}$

Acknowledgments

Table of Contents vi

$\begin{array}{ll}\text { List of Tables } & \text { ix }\end{array}$

List of Figures $\quad \mathbf{x}$

Nomenclature $\quad$ xiii

1 Introduction 1

1.1 The $\mathrm{LHC} \ldots \ldots \ldots \ldots \ldots \ldots \ldots$

1.2 The ATLAS detector $\ldots \ldots \ldots \ldots \ldots \ldots$

1.2.1 Inner Detector . . . . . . . . . . . . . . . . 6

1.2 .2 Calorimetry . . . . . . . . . . . . . . . 12

1.2 .3 Muon Spectrometer . . . . . . . . . . . . . . . . . . 14

1.3 The LHC Upgrade: Super LHC . . . . . . . . . . . . . . . . 14

1.3.1 ATLAS upgrades in preparation for the sLHC . . . . . . 15

2 Principles of semiconductor detectors $\quad 19$

2.1 Particle interactions with matter $\ldots \ldots \ldots \ldots$ 
2.1.1 Energy deposition by particles . . . . . . . . . . . 19

2.1.2 Energy deposition by photons .............. 23

2.2 Sensor mechanics . . . . . . . . . . . . . . . 25

2.2.1 Particle detection using semiconductors . . . . . . . . 25

2.2.2 Diamond as a sensor material . . . . . . . . . . 29

2.2.3 Production of synthetic diamonds . . . . . . . . . . . 31

2.3 Radiation damage ........................... 34

2.3.1 Increased leakage current . . . . . . . . . . . 36

2.3.2 Decreased charge collection distance . . . . . . . . . 38

3 ATLAS pixel modules $\quad 42$

3.1 Current generation sensing material . . . . . . . . . . . 43

3.2 Read-out electronics . . . . . . . . . . . . . . . 46

3.2.1 FE-I3 read-out electronics . . . . . . . . . . . . . 46

3.2.2 Digitizing hit information .............. . 49

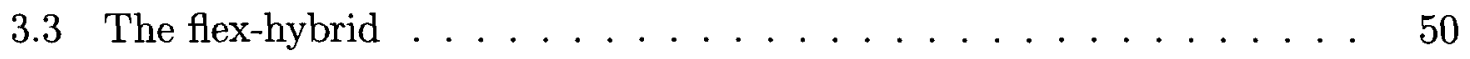

4 Characterization and Calibration of ATLAS Pixel Devices 52

4.1 The USBPix test system ...................... 53

4.2 USBPix test-stand hardware . . . . . . . . . . . . 54

4.3 Calibration of the front-end chip . . . . . . . . . . . 56

4.3 .1 Sensor Bias ...................... 56

4.3.2 Threshold Adjustment . . . . . . . . . . . . 57

4.3.3 ToT Adjustment .................... 59

4.3.4 Chip calibration algorithm ............. 67

4.4 Generating a pixel mask . . . . . . . . . . . . . 68

4.5 Source scans . . . . . . . . . . . . . . . . 69

4.5.1 Trigger Delay ................... 69 
4.5 .2 Results . . . . . . . . . . . . . . 70

5 Test-beam at CERN $\quad 78$

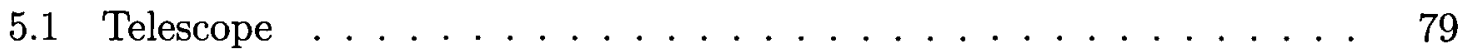

5.1 .1 Data acquisition . . . . . . . . . . . . 82

5.2 Dataset summary . . . . . . . . . . . . . . . . 83

5.3 Track reconstruction and alignment . . . . . . . . . . . . . 84

5.3 .1 Hit clustering . . . . . . . . . . . . . . 85

$5.3 .2 \quad$ Alignment . . . . . . . . . . . . . . . . 87

5.3 .3 Track fitting . . . . . . . . . . . . . . . . . . 90

5.4 Charge deposition . . . . . . . . . . . . . . . . 92

6 Summary 101

$\begin{array}{ll}\text { References } & 104\end{array}$ 


\section{List of Tables}

1.1 Upgrade schedule of the ATLAS detector . . . . . . . . . . . 17

2.1 Variables used in the Bethe-Bloch equation. . . . . . . . . . . . 21

2.2 Material properties of silicon and diamond . . . . . . . . 30

5.1 Summary of datasets taken and analyzed using scCVD diamond CD181. 84 


\section{List of Figures}

1.1 The constituents of the Standard Model . . . . . . . . . . . . 2

1.2 The LHC ring . . . . . . . . . . . . . . . . . . . 3

1.3 The ATLAS detector . . . . . . . . . . . . . . . . . . 6

1.4 Particle identification in the ATLAS detector . . . . . . . . . . . 7

1.5 The ATLAS inner detector . . . . . . . . . . . . . . 8

1.6 The ATLAS pixel detector . . . . . . . . . . . . . . . 10

1.7 Photos of the pixel detector central and end-cap assemblies . . . . . . 11

1.8 Simulated ATLAS events comparing pre and post upgrade tracks . . 16

$2.1 \mathrm{dE} / \mathrm{dx}$ for muons in copper . . . . . . . . . . . . . . . 20

2.2 Sample Landau distributions for $500 \mathrm{MeV}$ pions in silicon . . . . . . . 22

2.3 Depletion region at various bias voltages . . . . . . . . . . . 26

2.4 Example of a particle track passing through a semiconducting sensor. $\quad 28$

2.5 Schematic of pCVD and scCVD diamond pixel sensors. . . . . . . . 33

2.6 Photo of the ATLAS beam condition monitor . . . . . . . . . . 34

2.7 Energy level diagrams for different effects of radiation damage . . . . 36

2.8 Comparison of radiation damage from different particles as a function of energy . . . . . . . . . . . . . . . . . 37

2.9 Summary of proton irradiation results for pCVD and scCVD diamond 40

3.1 Photo of a full ATLAS pixel module with flex-hybrid. The sensing material cannot be seen in this photo. . . . . . . . . . . . . 43 
3.2 Simplified schematics of a full ATLAS pixel module . . . . . . . . . 44

3.3 Illustration of ganged pixels on an ATLAS full module board . . . . . 45

3.4 Analog circuit schematics of an FE-I3 pixel . . . . . . . . . . . 47

3.5 Graphical depiction of a time over threshold (ToT) determination . . 49

3.6 ATLAS pixel detector single-chip module . . . . . . . . . . . . . 51

4.1 USBPix test-stand setup . . . . . . . . . . . . . 53

4.2 Carleton University USBPix test-stand. . . . . . . . . . . . 55

$4.3 \mathrm{I}-\mathrm{V}$ curve for planar silicon chip 10-7A . . . . . . . . . . . 57

4.4 Pixel charge threshold mean for the globally tuned planar silicon FE-I3 chip 10-7A ..................... 60

4.5 Pixel charge threshold mean for the completely tuned planar silicon FE-I3 chip 10-7A . . . . . . . . . . . . . . 61

4.6 Pixel charge threshold noise for the globally tuned planar silicon FE-I3 chip 10-7A ......................... 62

4.7 Pixel charge threshold noise for the completely tuned planar silicon FE-I3 chip 10-7A ................... 63

4.8 ToT response for planar silicon FE-I3 chip 10-7A . . . . . . . . . . 65

4.9 ToT response for planar silicon FE-I3 chip 10-7A . . . . . . . . . . . 66

4.10 Cluster size distribution for Sr-90 source scan using planar silicon FEI3 chip 10-7A ...................... . . 71

4.11 Sr90 source scan hit-map. . . . . . . . . . . . . . . . . 72

4.12 Level 1 trigger distribution for Sr-90 source scan using planar silicon FE-I3 chip 10-7A .................. . . 73

4.13 To'T response for Sr-90 source scan using planar silicon FE-I3 chip 10-7A 75

4.14 Charge deposition for planar silicon FE-I3 chip 10-7A . . . . . . . . 76

5.1 Bonn-ATLAS Telescope scale image . . . . . . . . . . . . . . . 80

5.2 Overview of the TurboDAQ setup . . . . . . . . . . . . 82 
5.3 BAT hit read-out examples . . . . . . . . . . . . . . . 87

5.4 Sample alignment residual for BatMod $1 \ldots \ldots$. . . . . . 89

5.5 Alignment offset for BatMod3 (August 2007) . . . . . . . . . . . 91

5.6 Residuals between reconstructed hit locations in BatMod1 and fitted

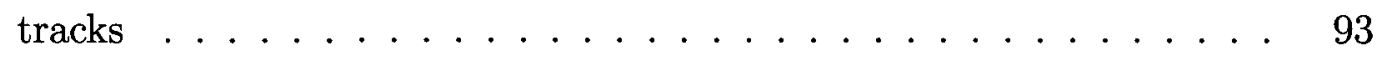

5.7 ToT distribution for October 2006 CD181 dataset . . . . . . . . . 94

5.8 ToT distribution for August 2007 CD181 dataset . . . . . . . . . 96

5.9 ToT distribution for the July 2008 CD181 dataset. Figure (a) shows the summary ToT distribution for the first 50 runs in dataset. Figure (b) shows the ToT distribution for every run. Charge collection properties can be derived from (a), while deviations in the MPV in later runs basis are clearly visible in (b). $30 \mathrm{BC}$ corresponds to $10 \mathrm{ke}$ deposited

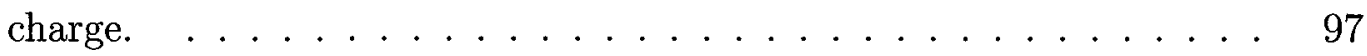

5.10 Evolution of ToT distribution over June 2009 CD181 test-beam runs . 98

5.11 Inverse charge collection distance vs irradiation in CD181 . . . . . 99 


\section{Nomenclature}

\section{Table of Abbreviations}

This thesis uses a number of commonly used abbreviations within the field of detector development; and in order to make the thesis more inclusive, a list of prevalent abbreviations can be seen below:

\begin{tabular}{cc}
\hline \hline Abbreviation & Definition \\
\hline CERN & European Center for Nuclear Research \\
LHC & Large Hadron Collider \\
sLHC & super Large Hadron Collider \\
ATLAS & A Torroidal LHC Apparatus \\
SPS & Super Proton Synchrotron \\
SCT & Semi-Conductor Tracker \\
TRT & Transition Radiation Tracker \\
MPV & Most probable value \\
MIP & Minimum Ionizing Particle \\
USBPix & Universal Serial Bus Pixel read-out electronics \\
\hline \hline
\end{tabular}




\begin{tabular}{|c|c|}
\hline Abbreviation & Definition \\
\hline $\mathrm{FE}-\mathrm{I} 3 / 4$ & Front End - Iteration 3/4 \\
\hline CVD & Chemical Vapor Deposition \\
\hline scCVD & single crystal Chemical Vapor Deposition \\
\hline $\mathrm{pCVD}$ & poly-crystal Chemical Vapor Deposition \\
\hline $\mathrm{MCC}$ & Module Controller Chip \\
\hline DAQ & Data Aquisition \\
\hline TLU & Trigger Logic Unit \\
\hline DAC & Digital to Analog Converter \\
\hline GDAC & Global threshold tuning register \\
\hline TDAC & Pixel threshold tuning register \\
\hline IF & Global feedback current tuning register \\
\hline FDAC & Pixel feedback current tuning register \\
\hline $\mathrm{BC}$ & Bunch Crossing ( $25 \mathrm{~ns}$ ) \\
\hline BCID & Bunch Crossing Identifier \\
\hline ToT & Time over threshold \\
\hline L1 or LVL1 & Level 1 trigger ID \\
\hline $\mathrm{EoC}$ & End of Column \\
\hline CS & Cluster Size (number of hits per reconstructed event) \\
\hline
\end{tabular}




\begin{tabular}{cc}
\hline \hline Abbreviation & Definition \\
\hline BAT & Bonn-ATLAS Telescope \\
BatMod & Bonn-ATLAS Telescope Module \\
DUT & Device Under Test \\
SiTBeAn & SiLab Test-Beam Analysis software \\
\hline \hline
\end{tabular}




\section{Table of Symbols}

This thesis uses a number of symbols which are application specific, and can be the cause of ambiguity. This list is provided to combat that ambiguity:

\begin{tabular}{|c|c|}
\hline Symbol & Definition \\
\hline$c$ & speed of light $\left(2.998 \times 10^{8} \mathrm{~m} / \mathrm{s}\right)$ \\
\hline$e$ & Electron charges $\left(-1.602 \times 10^{-19} \mathrm{C}\right)$ \\
\hline$R$ & ATLAS detector radial distance from beam-pipe \\
\hline$\phi$ & ATLAS detector polar angle \\
\hline$\theta$ & ATLAS angle between beam axis and position \\
\hline$\eta$ & ATLAS detector pseudo-rapidity coordinate \\
\hline$z$ & ATLAS detector cylindrical axis \\
\hline$E$ & Energy, usually in units of electron-Volts \\
\hline$\beta$ & Relativistic quantity $v / c$ \\
\hline$\gamma$ & Relativistic quantity, $E / m c^{2}$ \\
\hline$\frac{d E}{d x}$ & Energy deposition in a medium per unit length \\
\hline$\Delta_{p}$ & Landau most-probable value parameter \\
\hline$V_{b r a s}$ & Bias voltage applied to sensor \\
\hline$d$ & Width of the sensor's depleted region \\
\hline$E(x)$ & Electric field in a sensor \\
\hline
\end{tabular}




\begin{tabular}{cc}
\hline \hline Symbol & Definition \\
\hline$x$ & Position along the thickness of the sensor \\
$E_{e / h}$ & Energy to produce an electron hole pair \\
$\mu_{e / h}$ & Charge carrier mobility \\
$\Phi$ & Sensor radiation dose (particles/cm $\left.{ }^{2}\right)$ \\
$k_{r}$ & Irradiation damage constant \\
ccd & Charge collection distance \\
$I_{d}$ & Leakage current \\
$Q_{\imath n \jmath}$ & Injected charge into the sensor \\
\hline \hline
\end{tabular}




\section{Chapter 1}

\section{Introduction}

This thesis has two main goals: The first is to demonstrate that a new test stand for pixel devices is capable of properly calibrating and characterizing those devices. The other is to demonstrate that synthetic diamonds used in semiconducting pixel sensors are radiation tolerant under sLHC radiation conditions. This will be done by examining a sensor which has been tested at several levels of irradiation and characterizing the amount of charge trapping in the detector as a function of fluence. The goal is to demonstrate a tolerance on the order of, or better than, the existing silicon sensor standard. I will also outline my contributions to further efforts in the field of diamond sensor characterization.

This study was performed as part of a massive effort to further understand the nature of the universe, specifically the "Standard Model" of particle physics. The Standard Model is a theoretical model which describes the fundamental constituents and interactions of matter. This model has been under development since Ernest Rutherford first collided $\alpha$ and $\beta$ particles against stationary targets in 1911. The model states that matter is made up of spin- $\frac{1}{2}$ particles called fermions, which are divided into two groups called quarks and leptons. The model also describes integer spin particles, called bosons which mediate the fundamental forces. The complete list of particles can be seen in Figure 1.1. 


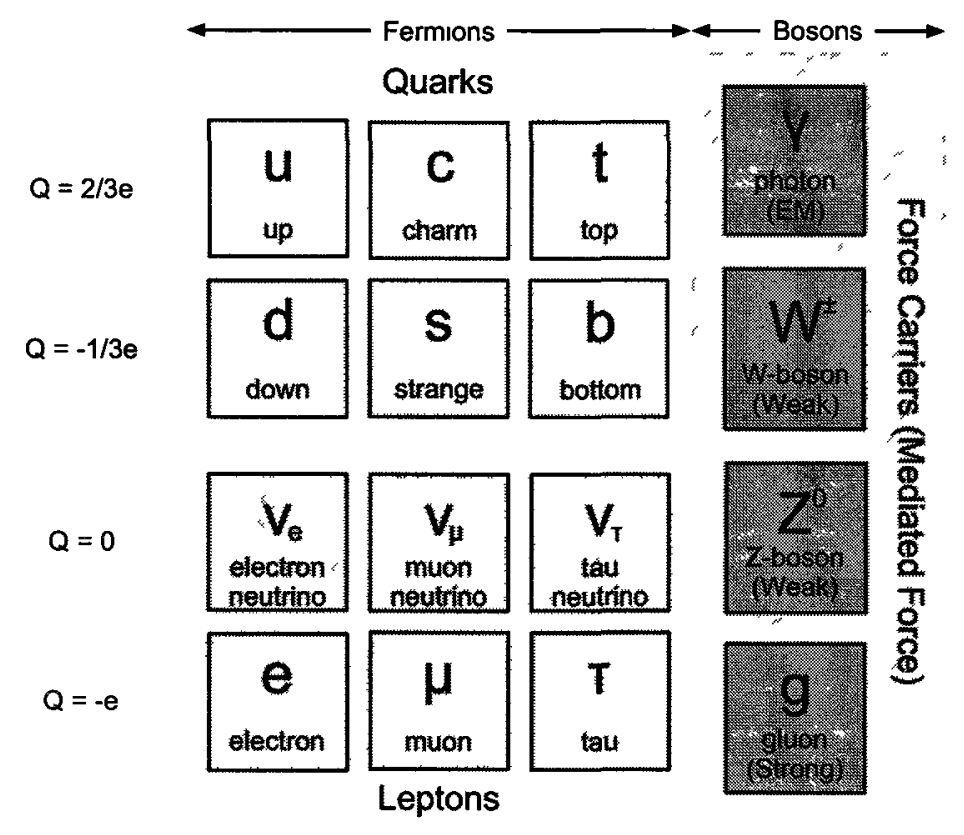

Figure 1.1: The constituents of the Standard Model

The Standard Model incorporates the electromagnetic, and the strong and weak nuclear forces with great accuracy, but does not include a description of gravity. There are also several other shortcomings to the model, the reason for matter/antimatter asymmetry, or an explanation of the origin of dark matter and energy, to list a few. There are several proposed theories such as supersymmetry (SUSY), technicolor, extra dimensions, or string theory which may resolve some of these issues.

A proposed solution to the origin of mass problem was introduced by Peter Higgs [1] which utilizes a spin-0 boson, dubbed the Higgs boson, and it remains the only particle in the Standard Model which has yet to be observed. Experiments such as the ATLAS experiment at the Large Hadron Collider (LHC) at CERN in Geneva, Switzerland have been designed specifically to find the Higgs boson, and any other unexpected phenomena that may appear at high energy. Thousands of scientists and engineers have spent nearly two decades developing new technology to overcome the difficulties of operating such a complicated machine. 


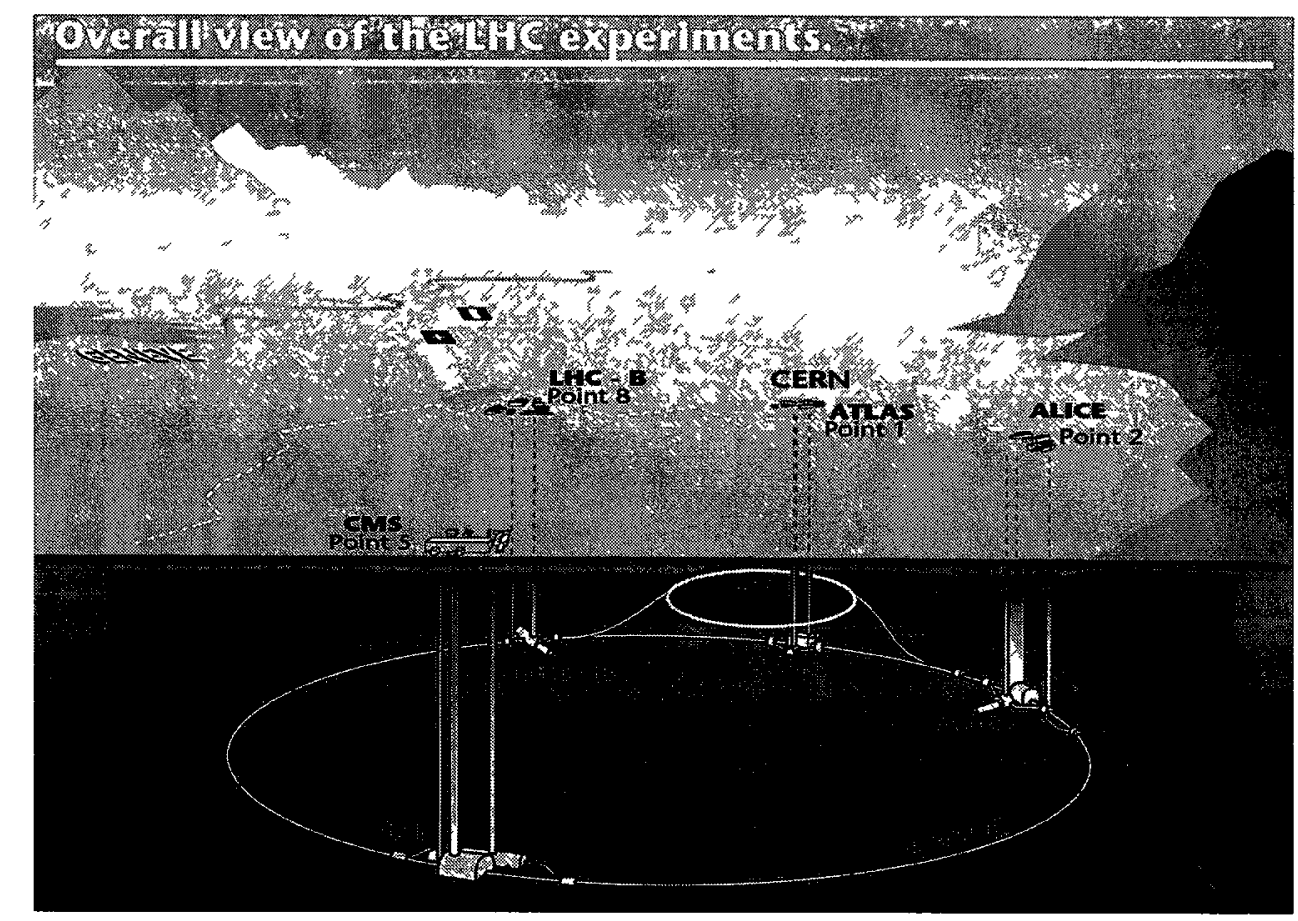

Figure 1.2: The LHC ring and major experiments [2] Used with permission

Particle collisions at the energies attanned in machmes such as the LHC produce temperatures and densities so high that they replicate conditions of the universe moments after the Big Bang. Very near the interaction point of the collision particles are produced which interact in ways which have not yet been reproduced in the lab. This gives scientists a controlled environment to look at these early moments of our universe, and understand how it has evolved into what it is now.

\subsection{The LHC}

The LHC is a $27 \mathrm{~km}$ long particle accelerator designed to collide bunches of protons at a center of mass energy of $14 \mathrm{TeV}$. It is located approximately $100 \mathrm{~m}$ under the FrenchSwiss border near Geneva, Switzerland. The device is designed to accelerate protons, but it is also capable of accelerating heavy ions such as lead for other experiments. 
The accelerator complex makes use of the existing $\mathrm{PS}^{1}, \mathrm{SPS}^{2}$, and $\mathrm{LEP}^{3}$ tunnels to accelerate 2808 bunches of $1.1 \times 10^{11}$ protons in stages.

The SPS is capable of accelerating proton bunches to $450 \mathrm{GeV}$ in as little as 4.3 seconds, but the time required to accelerate proton bunches in the LHC is on the order of 25 minutes after a 20 minute fill time. Proton bunches travel at a speed of $0.999999991 \mathrm{c}$, which results in bunch crossings and collisions every $25 \mathrm{~ns}$ and a designed instantaneous luminosity of $10^{34} \mathrm{~cm}^{-2} \mathrm{~s}^{-1}$.

The accelerator houses four main experiments which are exploring several areas of particle physics. The ATLAS and CMS detectors are general-purpose experiments designed to explore the high energy frontier in search of new physics. The LHCb experiment is designed specifically to shed light on physics involving the b-quark and matter-antimatter asymmetry. The ALICE experiment is a detector designed to observe the results of lead ion collisions at an energy of $1000 \mathrm{TeV}$ in an attempt to recreate a quark-gluon plasma which was hypothesized to exist in the very early universe.

Superconducting dipole and quadrupole magnets with a field strength of $8 \mathrm{~T}$ are used for bending and shaping the beam around its circular trajectory respectively. The bending magnets and accelerating $\mathrm{RF}$ fields are kept at $4.5 \mathrm{~K}$, but the focusing magnets are kept at $1.9 \mathrm{~K}$ to allow for stronger magnetic fields, and therefore tighter bunch packing. Two beams are kept in separate tubes and circulated in opposite directions around the ring. The two beams are focused to collide at the four interaction points of each experiment.

The first beam of the LHC was circulated in the fall of 2008 briefly before a catastrophic failure caused a complete shutdown of the accelerator for a year while the device was repaired and commissioned. Beam resumed in the winter of 2009 with

\footnotetext{
${ }^{1}$ Proton Synchrotron

${ }^{2}$ Super Proton Synchrotron

${ }^{3}$ Large Electron-Positron collider
} 
a decreased beam energy and luminosity to ensure the longevity and safety of the LHC. Collisions are currently occurring at a center of mass energy of $7 \mathrm{TeV}$, and a peak intantaneous luminosity of $3.9 \times 10^{30} \mathrm{~cm}^{-2} \mathrm{~s}^{-1}$ observed by ATLAS.

\subsection{The ATLAS detector}

ATLAS is an acronym for A Large Torroidal LHC Apparatus, and it is one of the general purpose particle detectors at the LHC designed to observe phenomena expected to occur at the $\mathrm{TeV}$ energy scale. It has over 3000 physicists including 1000 students as active members from 38 countries, making it one of the largest collaborative scientific efforts attempted in the physical sciences. For a more complete explanation of the ATLAS detector please consult [3].

The detector was designed to meet the requirements of observing evidence for some of the more promising theories, and these design principles were kept in mind:

- Ability to determine the presence of weakly interacting particles:

- Near full angle detector coverage.

- High efficiency energy/momentum reconstruction.

- Easy identification of particle interaction channels:

- High-resolution tracking.

- Easy particle identification.

- Ability to differentiate signal events from background events, and cope with a high data rate (trigger system).

- Ability to cope with the high-radiation and high-frequency environment produced by LHC collisions. 


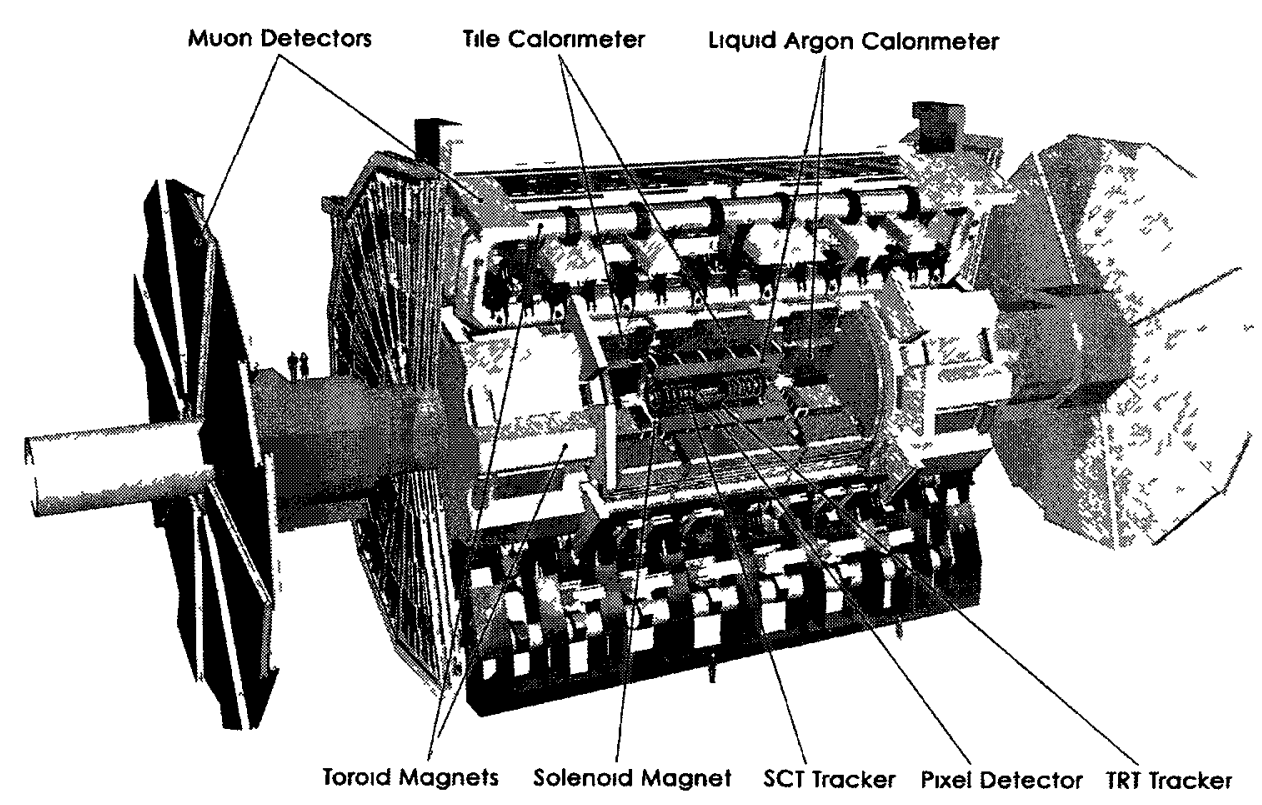

Figure 1.3: The interior of A Large Torroidal LHC Apparatus (ATLAS) detector with humans inserted into the image to assist with visualizing scale [2] Used with permission.

Figure 1.3 shows the layout of the ATLAS detector. ALTAS is an assembly of detectors, which perform separate tasks, in a magnetıc field. The inner detector reconstructs particle charge, position and momentum with the aid of the magnetic field, and the outer detector is devoted largely to energy reconstruction. Each part of the detector is divided into several sub-detectors which aid in particle identification, this effect can be seen in Figure 1.4. The barrel design of the detector ensures full angle coverage with the interaction point in the center.

As of August 2010, ATLAS has collected approximately $1.5 \mathrm{pb}^{-1}$ of data, the equivalent of over 100 billion collisions.

\subsubsection{Inner Detector}

The inner detector is located at the center of the ATLAS detector, and is approximately $1 \mathrm{~m}$ in radius from the beam line. It consists of a three layer pixel detector from 


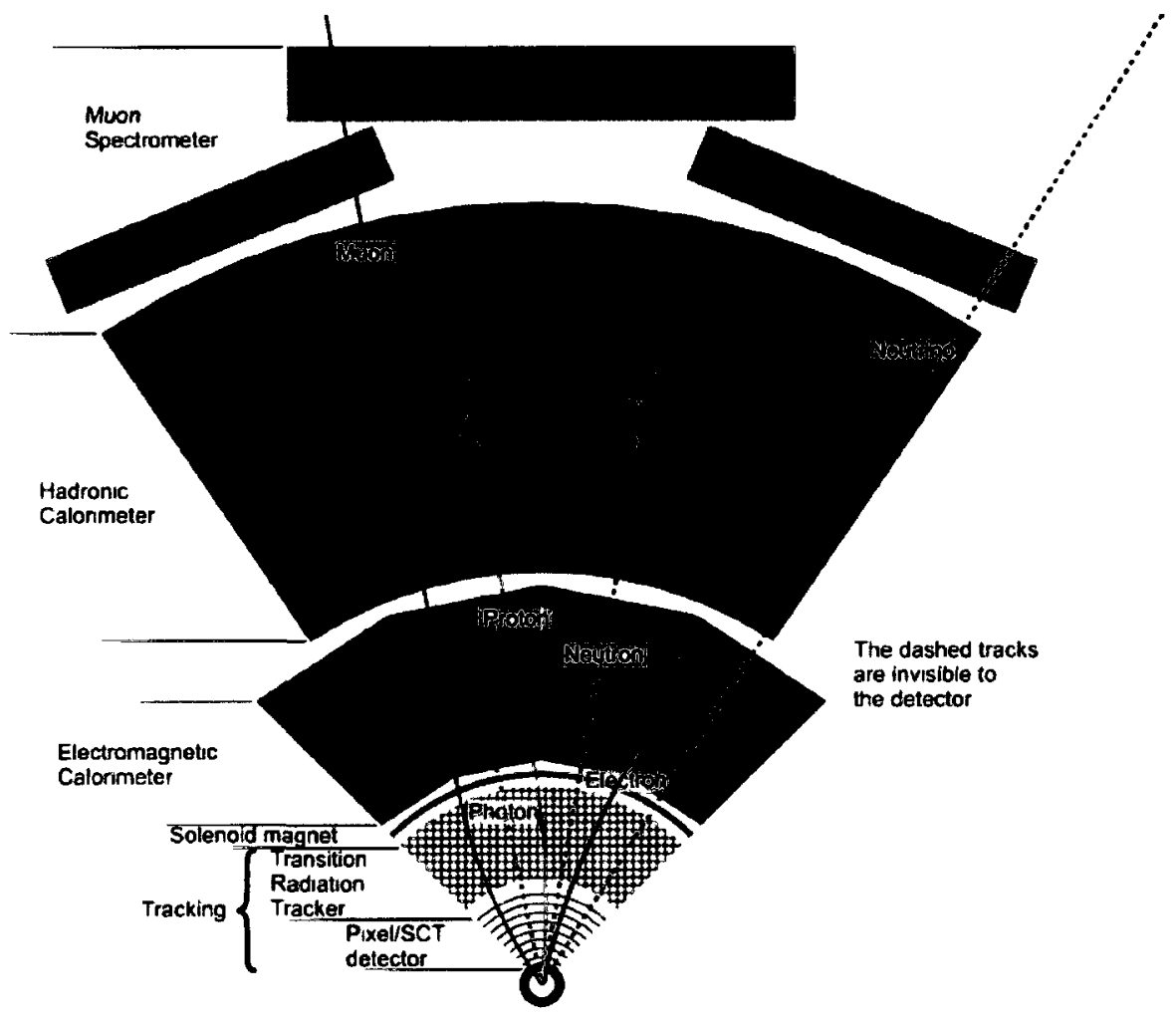

Figure 1.4: Particle identification in the ATLAS detector is done by identifying particle interaction signatures. Specific particles will only interact with certain detector components. When we combine our knowledge of which detector subsystems observed a particle with our knowledge of the magnetic field, we are able to reconstruct the charge, energy/momentum and position of all observed particles, and infer information about particles we do not observe [2]. Used with permission. 


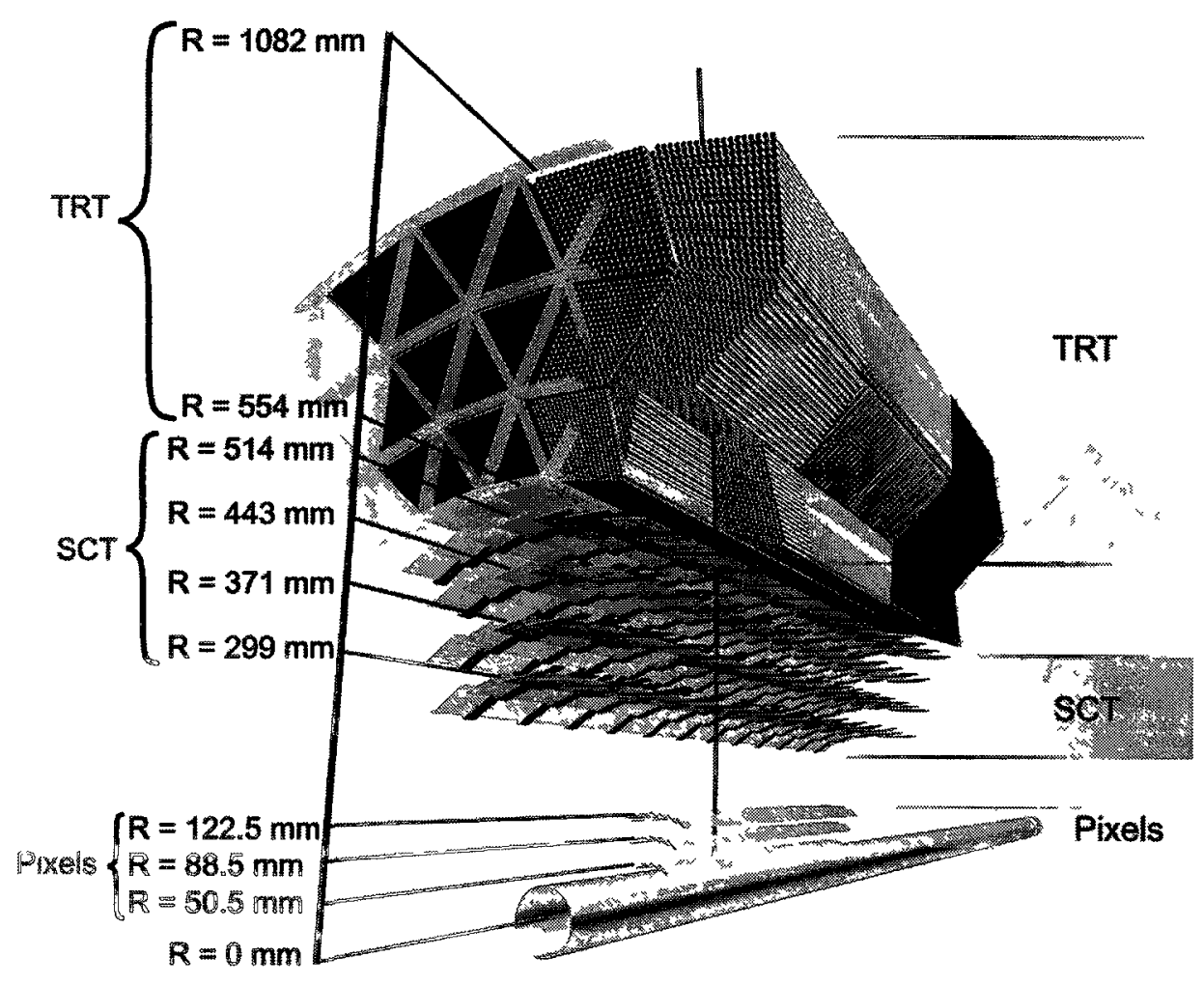

Figure 1.5: The ATLAS inner detector system, displaying the radial journey of a particle as it passes through the beryllium beam-pipe, and several tracking layers [2]. Used with permission.

$R=50.5 \mathrm{~mm}-122.5 \mathrm{~mm}$, four layers of strip detectors called the Semi-Conductor Tracker (SCT) from $R=299 \mathrm{~mm}-514 \mathrm{~mm}$, and a transition radiation tracker (TRT) from $R=554 \mathrm{~mm}-1082 \mathrm{~mm}$. The entirety of the inner detector is encased in a solenoidal $2 \mathrm{~T}$ magnetic field, and the layout can be seen in Figure 1.5.

The detector is designed to reconstruct the origin, trajectory, momentum and charge of charged particles created in the collisions at the heart of the ATLAS detector. The presence of charged particles is observed by their interaction with discretized detectors placed at specific points radially away from the interaction point, 
and cylindrically symmetric about the beam axis. The momentum of the particles is determined by observing the curvature of their trajectories in the magnetic field, and the relative charge of particles can be deduced based on the inflection of the particle trajectory's curvature.

\section{Pixel detector}

The density of tracks close to the interaction point in the detector drove the development of a silicon pixel detector. The active sensors are made of $250 \mu \mathrm{m}$ thick, radiation tolerant silicon. Pixels are $50 \times 400 \mu \mathrm{m}^{2}$ in size and have a resolution better than $15 \mu \mathrm{m}$ in the $R-\phi$ direction, and better than $120 \mu \mathrm{m}$ in the direction of the beam-axis.

Because the pixel detector is capable of delivering high-accuracy tracking in 3 spatial dimensions, it can substantially contribute to track-finding algorithms and assists in the identification of secondary vertices. The detector has 3 cylindrical layers surrounding the beam-pipe, and 3 disks closing each end-cap. The pixel detector has over 80 million read-out channels (one for each pixel), which is approximately half of the read-out channels for the entire detector. A computer generated image of the pixel detector and its support structure can be seen in Figure 1.6.

In the central region of the detector 3 barrels are located at $R=$ $50.5,88.5$ and $122.5 \mathrm{~mm}$, and they provide coverage of tracks with $|\eta|<1.9^{4}$. The size of the beam-pipe is the current limiting factor when considering where to place the first layer of pixel detectors.

An additional pixel layer called the insertable beam layer (IBL) has been proposed to provide hit reconstruction abilities closer to the interaction point. These extra

\footnotetext{
${ }^{4} \eta$ represents pseudo-rapidity - an angular scale which denotes the angle $\theta$ between the beam axis and the particle trajectory where $\eta=-\ln (\tan (\theta / 2))$. This scale was chosen because it gives more precision on the parts of the detector which are covered by the pixel detector, while diverging to infinity close to $\theta=0^{\circ}$ where there is no pixel coverage.
} 


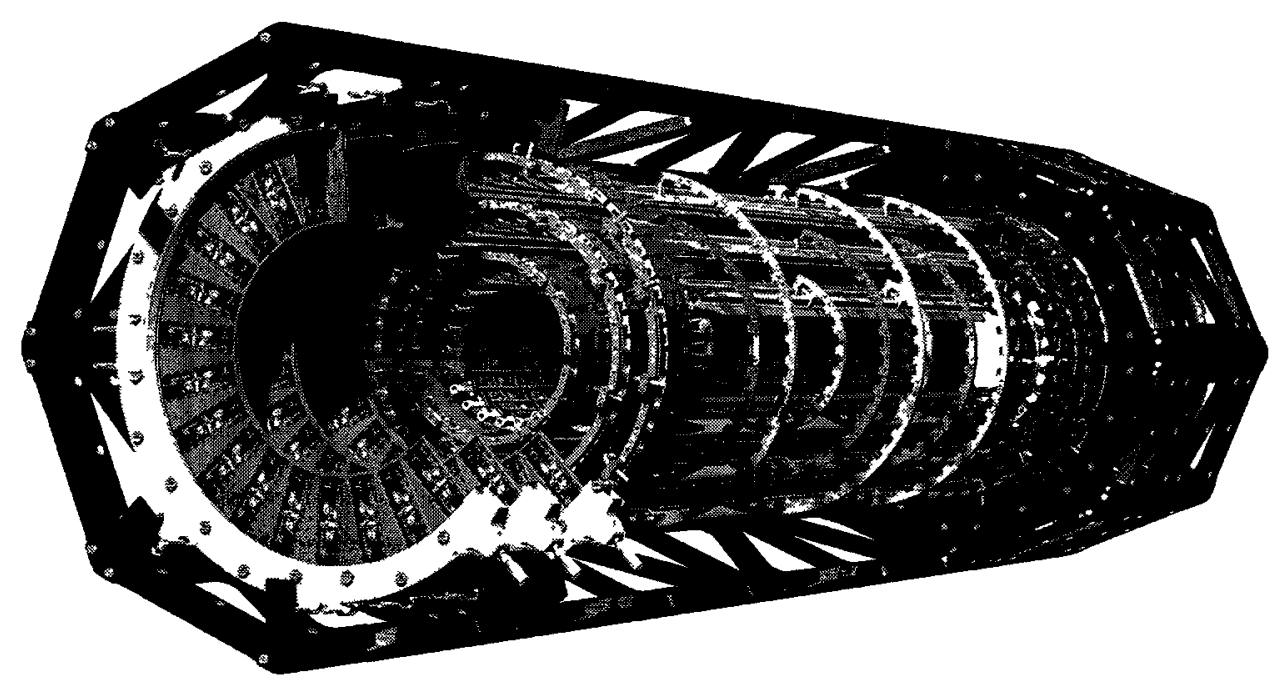

Figure 1.6: The ATLAS pixel detector, including support structure [2]. Used with permission.

layers would be inserted at $R=49.5,58$ and $65 \mathrm{~cm}$, and will increase ATLAS' sensitivity to secondary vertices near the interaction point - a region which is critical in the study of new physics.

The sensitive area of the pixel detector is made up of detector modules measuring approximately $2 \times 6 \mathrm{~cm}^{2}$. Modules are mounted in a layout similar to that of shingles on a roof to ensure there are no dead areas between modules at an angle of $-20^{\circ}$ with respect to the cylinder axis. Modules on the end-cap are mounted with each cap rotated out of phase with respect to the others for the same reason. Photos of the central and end-cap assemblies can be seen in Figure 1.7.

The discussion of the pixel detector has been expanded relative to the other detector systems due to the main focus of this thesis being in the upgrade of the pixel detector. The details of the pixel detector modules will be discussed in chapter 3 .

\section{Semi-Conductor Tracker (SCT)}

The SCT is similar in design to the Pixel detector. It contributes to tracking information with 4 barrel layers, and 9 end-cap disks on either side of the interaction point. 


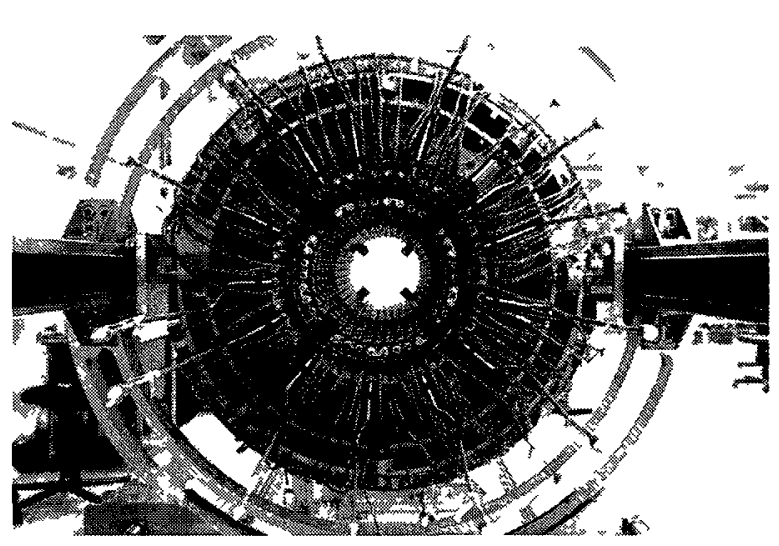

(a) End-on photo of pixel barrel region assembly

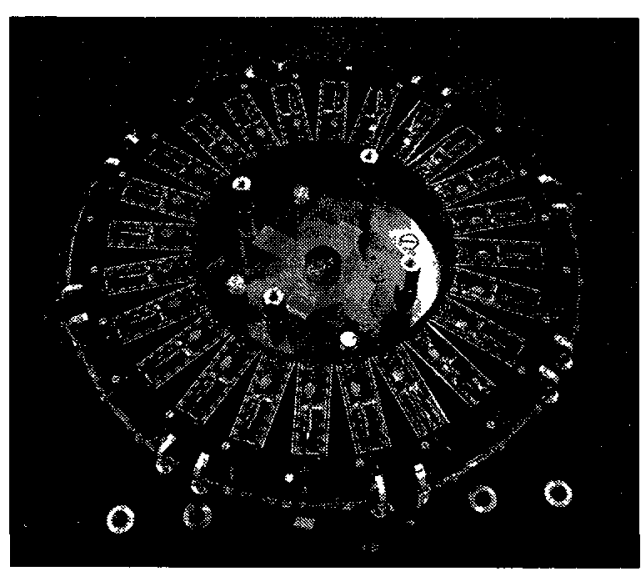

(b) Photo of pixel end-cap assembly

Figure 1.7: Photos of the pixel detector central and end-cap assemblies [2]. Used with permission.

Each layer consists of two single sided p-in-n silicon strip detectors, which are glued together at a stereo angle of $\pm 40 \mathrm{mrad}$ relative to the geometrical center of the sensors to give a 2 dimensional spatial sensitivity of $17 \mu \mathrm{m}$ in the lateral $(R-\phi)$ direction and $580 \mu \mathrm{m}$ in the longitudinal ( $R$ or $z$ ) direction. Strip width varies depending on the location of the module, but it is typically $80 \mu \mathrm{m}$ in the barrel, and between 57 and $94 \mu \mathrm{m}$ in the end-caps. The active area of each sensor is $300 \mu \mathrm{m}$ in thickness and $6 \times 6 \mathrm{~cm}^{2}$ on the normal plane.

\section{Transition radiation tracker $(\mathrm{TRT})$}

The TRT is similar in design to the SCT and pixel detectors in that it is a barrel in the central region, with disks on the end-caps. The detector consists of tubes with a $4 \mathrm{~mm}$ radius oriented along the beam axis in the barrel, and in the detector's radial direction in the disks. The tubes vary in length between 40 and $150 \mathrm{~cm}$ depending on their location in the detector, and are filled with a $\mathrm{Xe}-\mathrm{CO}_{2}-\mathrm{O}_{2}$ (70:27:3) gas mixture, with a $30 \mu \mathrm{m}$ wire running down the center of the tube. The area between each tube 
is filled with synthetic fibers (in the barrel) or foil-stacks (in the disks) which release transition radiation at every material to air transition.

This transition effect is mainly present for high- $\gamma$ electrons and positrons, and this makes it very useful for particle identification at energies where time of flight and Cerenkov radiation are not an option. The detection of transition radiation in addition to a track is used to distinguish between electrons and positrons (which produce transition radiation at approximately $1 \mathrm{GeV}$ ) and other higher-mass particles such as pions (which produce transition radiation at around $100 \mathrm{GeV}$ ).

The $\mathrm{Xe}$ in the gas mixture interacts with these transition photons and produces signal which is collected on the wires at both ends of the detector. The distance of a particle to the wire can be determined with a spatial sensitivity of $170 \mu \mathrm{m}$, but has no sensitivity to the position of the particle along the tube in the axial direction.

\subsubsection{Calorimetry}

Energy reconstruction in particle physics is done using calorimeters which contain many sub-detectors to assist in the identification and reconstruction of particles with as high an efficiency as possible. This is done by both absorbing the energy deposited into the calorimeter and sampling the shape of particle showers produced in the calorimeters.

The calorimeters are divided into electromagnetic and hadronic sub-detectors. The detectors are designed in such a way that particles which interact mainly electromagnetically such as photons and electrons are stopped within the electromagnetic calorimeter, and hadrons, which produce much larger cascades of particles will deposit energy in the hadronic calorimeter via collisions with the nuclei of the absorbing material. Only long-lived muons and weakly interacting particles such as neutrinos will completely escape the detector. 


\section{Electromagnetic Calorimeter}

The electromagnetic calorimeter uses lead and stainless steel absorbers, and liquid argon (LAr) is used to sample the shape of the shower. LAr acts like a dense gas and will ionize in the presence of particle showers producing a signal used in readout, and is resistant to radiation damage and the production of free radicals because it is a noble gas. The electromagnetic calorimeter must be placed in cryogenics to keep the argon at a liquid state. The absorbers and electrodes of the detector were designed in the shape of an accordion (saw-tooth) for a full coverage in $\phi$ with no cracks. The detector is capable of energy reconstruction on the order of $10 \% / \sqrt{E}+0.17 \%$ (where $E$ is in $\mathrm{GeV}$ ), and can determine the angular location of energy deposits with an accuracy of 0.025 radians in both $\theta$ and $\phi$.

\section{Hadronic Calorimeters}

The hadronic calorimeter is placed behind the electromagnetic calorimeter, and the forward region consists of a tile calorimeter in the barrel region, and a forward calorimeter (FCal) and a hadronic end-cap calorimeter (HEC) in the end-caps. The tile calorimeter uses a steel absorber and a polystyrene scintillator doped with wavelength-shifting fluors as the active medium. Particles that reach the scintillator produce UV scintillation light, which is converted to visible light by the doping chemicals.

The FCal is a LAr calorimeter which uses copper absorbers in the first electromagnetic layer, and tungsten absorbers in the second and third hadronic layers. The HEC uses copper absorbers while also using LAr as the active medium. The combined fractional energy resolution for the hadronic calorimeter was determined to be $52 \% / \sqrt{E}+3 \%[3]$ ( $E$ is again in $\mathrm{GeV})$. 


\subsubsection{Muon Spectrometer}

The muon spectrometer is designed to detect particles leaving the barrel and end-cap calorimeters, measure the momentum of particles in the $|\eta|<2.7$ range, and trigger on particles which have tracking information in the $|\eta|<2.4$ range. Since muons are essentially the only charged particle long-lived enough to reach the outside of the detector, the spectrometer is an excellent way to identify physics channels of interest.

Muon energy is measured by detecting the deflection of muons in the toroidal magnetic field, which requires the track of a muon to be measured with a precision on the order of $80 \mu \mathrm{m}$. The toroidal field is produced by 8 superconducting loops in the barrel region, and separate magnets in each end-cap. Muons are detected by monitored drift tubes in the barrel region, and cathode strip chambers in the end-

caps. Both chamber types can be read out in 15-25 ns providing bunch crossing identification capabilities to the detector, and can read out hit information in the bending $\eta$-axis and non-bending $\phi$-axis.

\subsection{The LHC Upgrade: Super LHC}

Continued operation of the LHC will produce a plethora of data in the new energy regime, but the data collected will serve as a platform for physicists to decide the direction of future LHC operations. The luminosity of the LHC will eventually reach a saturation point, where continuing to collect data at the existing rate will not significantly improve the statistics of existing data. To prepare for this eventuality, the CERN has begun preparation for an LHC upgrade dubbed the Super LHC (sLHC).

When looking for new physics there are three main considerations one takes when designing an accelerator: Particles collided, operational energy and luminosity. The type of particles collided and the operational energy of an accelerator dictate what reactions are possible and the fraction of times those reactions will occur per collision. 
The luminosity of the collider dictates the frequency with which collisions occur, and is therefore responsible for the rate at which data is collected from the experiment.

Changing the colliding particles or increasing the energy of the experiment is not an easy task. Increases in operational energy require stronger magnets to contain the beam within the existing beam-pipe, and changing the type of particles collided would change the energy required to accelerate the particles around the ring, potentially to a level beyond the capabilities of the accelerator. Changes to the energy and collisions of the beam could also require a complete redesign of the detectors, which were designed to exploit certain operational parameters of the collisions produced in the accelerator.

Changing the luminosity of the beam increases the number of particle collisions per bunch crossing, and will subsequently increase the number of particles per event. This creates an environment which is densely populated with high energy particles, and poses a severe danger to the longevity of exposed detectors. The LHC collaboration has begun the process of increasing the instantaneous luminosity of the LHC by a full order of magnitude to $10^{35} \mathrm{~cm}^{-2} \mathrm{~s}^{-1}$.

An ATLAS survey suggests an LHC luminosity upgrade could significantly benefit new physics searches at the limit of LHC sensitivity. [4]

\subsubsection{ATLAS upgrades in preparation for the sLHC}

Detector components in ATLAS are all qualified for 10 years of use in an LHC environment, but some components will likely not be qualified for 10 years of use in the sLHC environment while others will not be viable at all. An increase in luminosity exposes all sub-detectors in the ATLAS detector to an increase in the density of high energy particles. The number of collisions per bunch-crossing is expected to increase 80-fold per collision as illustrated in Figure 1.8. While the upgrade would ideally require as few changes to the existing detector as possible, some sub-detectors will require drastic changes in order to cope with this increase in particle flux and energy 


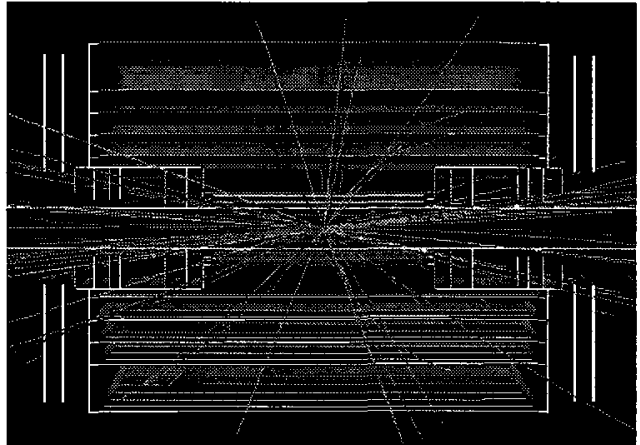

(a) 5 Simulated collision (equivalent $0.2 \times$ $\left.10^{34} \mathrm{~cm}^{-2} \mathrm{~s}^{-1}\right)$.

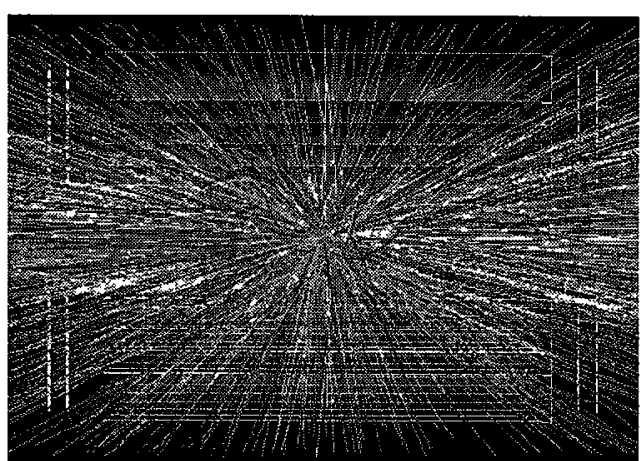

(b) 400 Simulated collision (equivalent to $\left.10^{35} \mathrm{~cm}^{-2} \mathrm{~s}^{-1}\right)$.

Figure 1.8: Simulated ATLAS events comparing pre and post upgrade tracks [5]. Used with permission.

deposition.

The inner detector is especially susceptible to the new environment, and will need to be replaced entirely. The pixel and SCT detectors will exhıbit decreased performance due to radiation damage, and the TRT will be rendered useless by the prohibitively high hit occupancy. Finding radiation-hard sensors is a top priority for the $R \& D$ groups, while designing a new tracking system that would operate within the constraints of the existing ATLAS detector.

The pixel detector is currently researching upgrades in both its read-out electronics and sensing materials. Read-out electronics are being designed with new radiationtolerant layouts and redundancies to survive in the sLHC environment as well as to increase the active area of the detector six fold while decreasing the size of individual pixels. The new electronics must also deal with high instantaneous rate, which can cause a pile-up of tracks. Small prototypes of these electronics have been validated to 200 Mrad [6], but much work remains before they will be ready for use in the ATLAS detector.

There are several candidate sensing materials being researched which can maintain 
the levels of performance required for track reconstruction after irradiation. The sensor $R \& D$ groups are working on variety of materials and silicon layouts. RD50 [7] works on developing radiation tolerant materials and layout using silicon, RD39 [8] is developing super-radiation hard cryogenic silicon detectors, and RD42 [9] is studying the use of electronic grade diamonds as a sensing material. The groups are currently researching the properties and performance of these sensors to determine their viability in the ATLAS detector.

While the sensor R\&D groups are not limited to the upgrade schedule of the LHC or any of the individual LHC experiments, there is a large push on the groups to develop these sensor materials for use in the upgrade. The schedule for the ATLAS upgrade is outlined in Table 1.1.

\begin{tabular}{ll}
\hline \hline Milestone & Date \\
\hline LHC shutdown to go to $14 \mathrm{TeV}$ & 2012 \\
New Insertable B-layer installation & Ready: 2014, Install: 2016 \\
Technical proposal for upgrade & 2013 \\
Detector production & 2015 \\
Stop LHC & late 2019 \\
Remove old detectors, install new ones & 2020 \\
Commission new detectors & 2021 \\
Take data & Mid-2021 \\
\hline \hline
\end{tabular}

Table 1.1: Upgrade schedule of the ATLAS detector. [5]

Upgrades to the read-out electronics and sensing material are both required in order for the inner detector to survive in the sLHC. Without this upgrade, the ATLAS detector would be without tracking information, and therefore without a critical component of event reconstruction. These upgrades will ensure the ATLAS experiment is able to perform at the forefront of experimental physics research. 
The topic of this thesis is to outline the contributions made to studying diamond sensors and their suitability as a next generation pixel detector sensing material, and the steps taken to bring Carleton University to the forefront of sensor characterization. The principles behind semiconducting sensor operation, properties of diamonds as a sensing material, and the effects radiation damage has on their performance is outlined in chapter 2. Chapter 3 introduces ATLAS pixel assemblies and explains the operation of the read-out electronics to the point of sensor operation.

Chapter 4 outlines a calibration system I established at Carleton University, and a sample device calibration I performed. I demonstrate the calibration capabilities and performance limitations of the test stand. I characterized a prototype diamond sensor for use in a particle beam test at CERN using a similar calibration software suite. I was responsible for data collection and quality management during several SPS beam tests, and took on the responsibilities of an on-call expert for the pixel detector beam-tests.

I have developed event selection, device alignment, tracking and charge collection analysis software to analyze the data taken in those beam tests. This code has been adopted by the diamond pixel data-analysis group, and is being modified to increase track reconstruction accuracy, and obtain a resolution measurement from the diamond devices. The analysis procedures and a demonstration of the software's capabilities are outlined in chapter 5 , along with a simple analysis of sensor performance.

Chapter 6 summarizes the results I obtained in the development of the Carleton test-stand, and discusses my observations about the data obtained in the beam-tests made with the new analysis software while comparing them to a previous analysis framework. 


\section{Chapter 2}

\section{Principles of semiconductor detectors}

Particles are detected and identified by observing how they interact with matter. Energy deposition in matter is different for photons, charged particles, and neutral particles. This chapter will outline how particles interact with matter, and specifically how energy deposition into semiconducting materials is used to generate charge carrier pairs, and signal pulses which are processed by specialized read-out electronics.

\subsection{Particle interactions with matter}

The subject of particle interactions while passing through a bulk material is of utmost importance to the development of particle detectors. A more complete treatment of these principles is available in [10].

\subsubsection{Energy deposition by particles}

The stopping power of a material for a given particle is typically measured by a quantity called $\langle d E / d x\rangle$, which is parametrized by the density of intermediate material, and the velocity of the incident particle (often expressed as $\beta \gamma=p / m c$, where $p$ and $m$ are the momentum and mass of the particle in question and $c$ is the speed of light). The intermediate material's atomic mass and charge, as well as the charge 


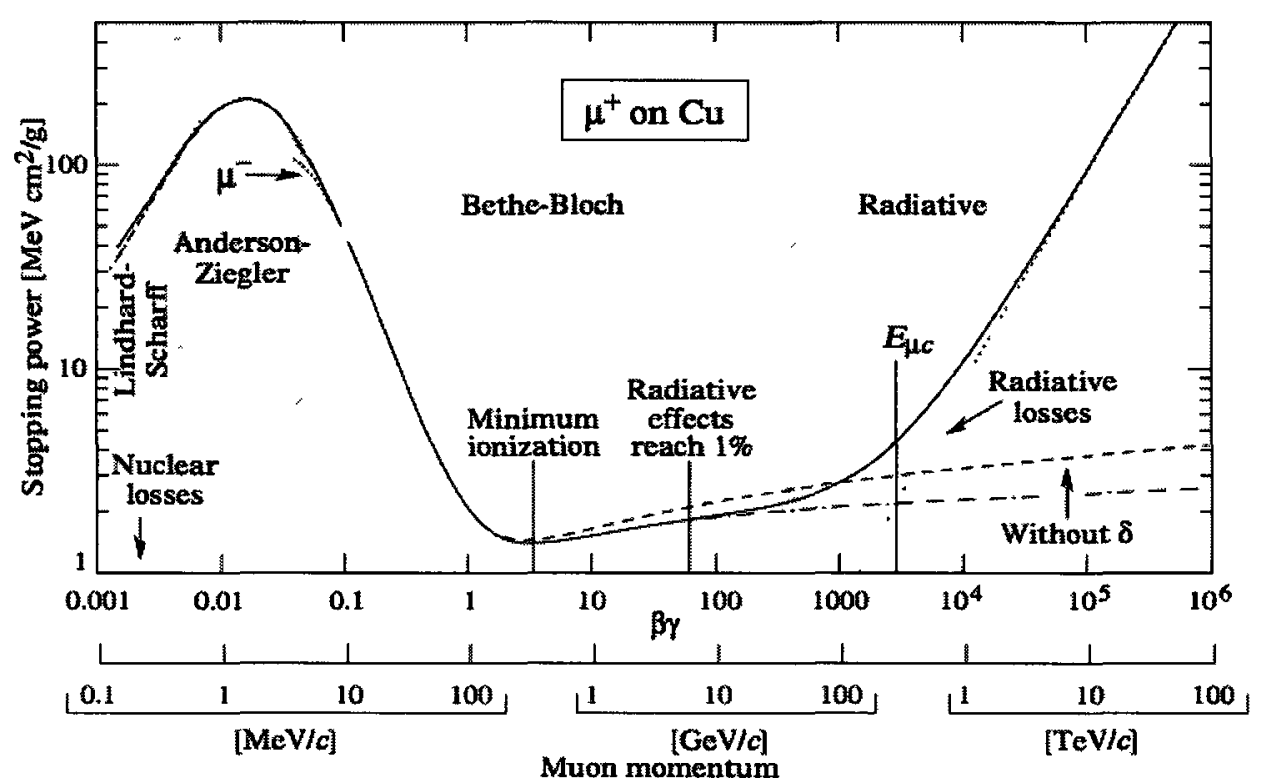

Figure 2.1: $\mathrm{dE} / \mathrm{dx}$ for muons in copper [10]. Used with permission.

of the incident particle also play a role in the magnitude of energy deposited in the material. An example of stopping power as a function of $p$ can be seen in Figure 2.1 for $\mu^{+}$particles passing through copper.

Energy loss for particles with velocities less than $\mathcal{O}(0.01 c)$ is dominated by electronic stopping power, and were successfully described by Lindhard [11]. Highvelocity particles $(1000<\beta \gamma)$ are dominated by radiative effects. Most interactions in high energy particle physics occur in the $0.1<\beta \gamma<1000$ range, and this region of the $d E / d x$ curve is best described by the Bethe-Bloch equation [10]

$$
\left\langle\frac{d E}{d x}\right\rangle=-K z^{2} \frac{Z}{A} \frac{1}{\beta^{2}}\left[\frac{1}{2} \ln \frac{2 m_{e} c^{2} \beta^{2} \gamma^{2} T_{\max }}{I^{2}}-\beta^{2}-\frac{\delta(\beta \gamma)}{2}\right] .
$$

The variables used in equation 2.1 are described in Table 2.1. This equation maintains accuracy within a few percent for particles where $0.1<\beta \gamma<1000$. The dominant source of energy loss for particles in this range is ionization. Corrections exist at low and high velocities to better match experimental results, such as the 
Anderson-Ziegler correction at low velocities [12] and the density effect which corrects energy deposition discrepancies between solids and gasses at higher velocities [13].

Ionizing radiation which deposits the minimum amount of energy in the absorbing material is said to be a minimum ionizing particle (MIP). Energy deposition for MIPs is typically on the order of $1.5 \mathrm{MeV} \cdot \mathrm{cm}^{2} / \mathrm{g}$ for nearly every type of absorbing material. The increase in energy deposition for particles with higher velocities is very small, making the energy deposited by MIPs a reasonably good approximation of energy deposition until radiative effects begin to play a non-negligible role in the calculation.

\begin{tabular}{lll}
\hline \hline Symbol & Definition & Units or Value \\
\hline$M$ & Mass of incident particle & $\mathrm{MeV} / \mathrm{c}^{2}$ \\
$E$ & Energy of incident particle & $\mathrm{MeV}$ \\
$T$ & Kinetic energy of incident particle & $\mathrm{MeV}$ \\
$m_{e}$ & Electron mass & $0.510998918(44) \mathrm{MeV}$ \\
$r_{e}$ & Classical electron radius $e^{2} /\left(4 \pi \epsilon_{0} m_{e} c^{2}\right)$ & $2.817940325(28) \mathrm{fm}$ \\
$N_{A}$ & Avogadro's number & $6.0221415(10) \times 10^{23} / \mathrm{mol}$ \\
$z$ & Charge of incident particle & \\
$Z$ & Charge of intermediate material & \\
$A$ & Atomic mass of intermediate material & $\mathrm{g} / \mathrm{mol}$ \\
$K$ & $4 \pi N_{A} r_{e}^{2} m_{e} c^{2}$ & $0.307075 \mathrm{MeV} \cdot \mathrm{cm}^{2} / \mathrm{g}$ \\
$I$ & Mean excitation energy & $\mathrm{eV}$ \\
$\delta(\beta \gamma)$ & Density effect correction & \\
\hline \hline
\end{tabular}

Table 2.1: Variables used in the Bethe-Bloch equation.

The first derivation of an energy loss distribution for a particle in a thin detector was done by Landau [14]. The function is characterized by the most probable energy deposition $\left(\Delta_{p}\right)$ and the full width of the distribution at half maximum $(w)$. Because the energy deposition depends heavily on the medium material and thickness of the 


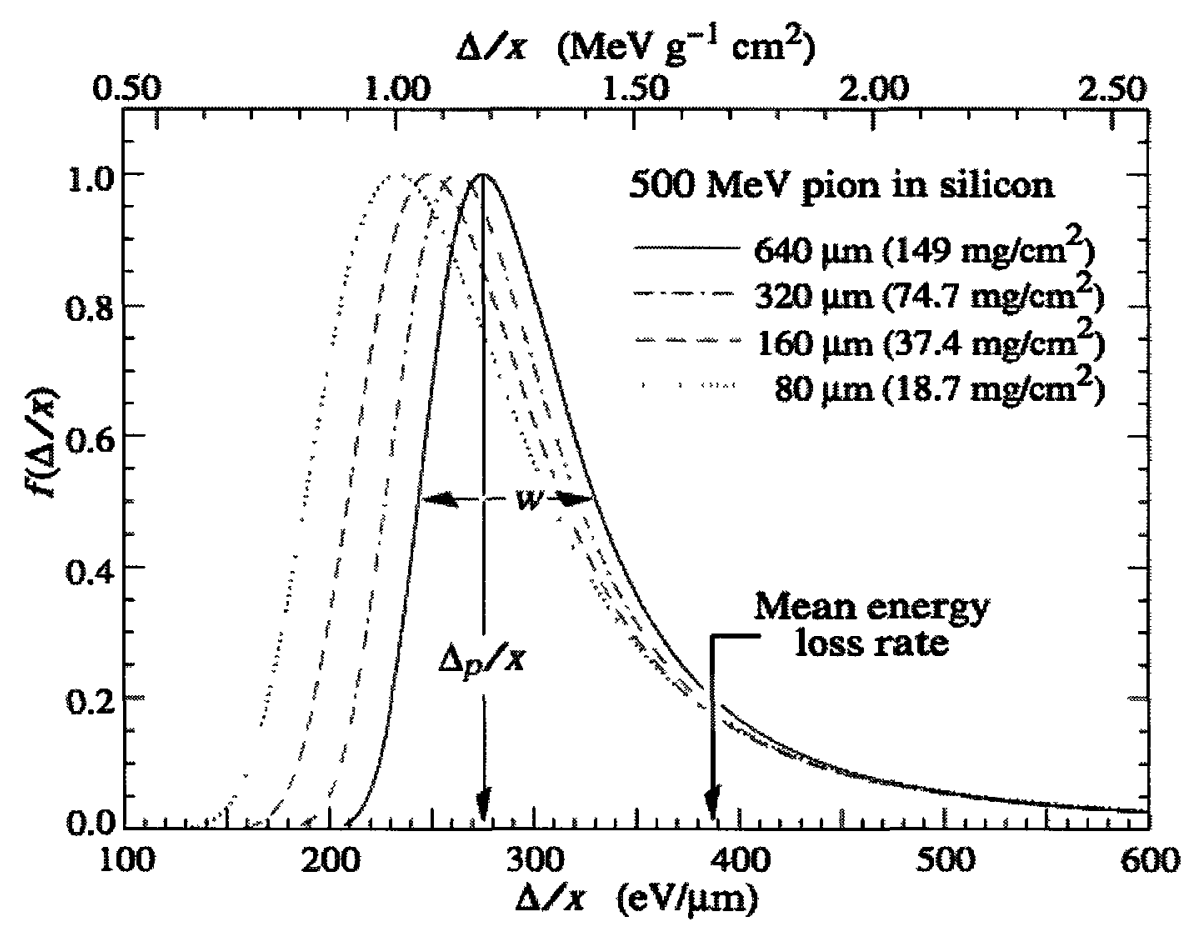

Figure 2.2: Straggling or Landau Distributions for $500 \mathrm{MeV}$ pions in silicon, normalized to unity at $\Delta_{p} / x$. Full width at half maximum is labeled $w$ on the graph [10]. Used with permission.

detector, accurate determinations of these parameters require careful consideration. Thin sensors increase the width of the distribution, while thicker sensors converge the distribution with a gaussian. The parameters of a Landau distribution are easily measured in experiment, and a fully labeled example of a Landau distribution can be seen in Figure 2.2.

An accurate study of energy deposition in silicon was performed Bichsel [13], who considered the material dependence of momentum transfers in the medium as well as how the number of collisions is affected by the thickness of the sensor. It was shown that the parameters of energy deposition for particles with $\beta \gamma>100$ in a sensor of thickness $d$ in $\mu \mathrm{m}$ are given by 


$$
\begin{array}{r}
\Delta_{p}=d \cdot(190+16.3 \cdot \ln d), 110<d<3000 \\
w=d \cdot(259.6+28.4 \cdot \ln d), 30<d<260 \\
=71.3 \cdot d \cdot\left(1+39.4 \cdot t^{-08}\right), 260<d<3000
\end{array}
$$

where both $\Delta_{p}$ and $w$ are in $\mathrm{eV}$. Another major factor of energy loss in matter is Bremsstrahlung radiation. Brem photons are generated as a particle passes through matter, and the energy emitted by the incident particle is given by

$$
-\left\langle\frac{d E}{d x}\right\rangle_{\text {brem }}=E \frac{Z(Z+1)}{A} \ln \frac{287}{Z^{\frac{1}{2}}} \frac{\rho}{716.4 \mathrm{~g} \cdot \mathrm{cm}^{-2}} \equiv \frac{E}{X_{0}},
$$

where $X_{0}$ ss defined as the radiation length of a sensor. $X_{0}$ is a material property which parametrizes energy deposition in the sensor, and is useful in describing multiple scattering and pair production. The contributions of these photons to energy deposition in the sensor is negligible because they are rarely reabsorbed within the thickness of a low $Z$ semiconducting sensor.

\subsubsection{Energy deposition by photons}

Silicon sensors are capable of detecting elecromagnetic radiation from frequencies in the infrared to X-rays. Photons interact with sensors by three main channels: the photoelectric effect, the Compton effect, and pair production [10]. In the case of the photoelectric effect and pair production the photon is absorbed entirely by the sensor, and in the case of the Compton effect the photon is scattered at a large angle.

$$
I(x)=I_{0} \exp -x / \mu
$$

where $I_{0}$ is the initial intensity of the beam, $x$ is the distance a beam has travelled 
through a sensor, and $\mu$ is a property of the sensing material called the attenuation length which also depends on the energy of the photon absorbed. The photon absorption probability for a $300 \mu \mathrm{m}$ thick sensor is shown in Figure ?? as a function of photon energy.

For photons with energy $E_{\gamma}<0.1 \mathrm{MeV}$, absorption is dominated by the photoelectric effect which depends on the atomic charge of the atom to the power of 4 or 5 depending on the photon energy. Photons absorbed via the photoelectric effect will be fully transferred to an electron of an atom. For photon energies near the bandgap, an electron-hole pair will be created; for energies much higher than the bandgap however, a free electron will be created which will create new electron-hole pairs via the methods described in the previous section.

In the $0.1 \mathrm{MeV}<E_{\gamma}<10 \mathrm{MeV}$ range Compton scattering takes over. Because the energy deposited in the sensor is continuous, the number of electron-hole pairs produced from a photon of fixed energy also varies. The variance of electron-hole pairs created by Compton scattering makes this energy range less useful than those created from the photoelectric effect for testing purposes.

Electron-positron pair production dominates absorption above photon energies of $10 \mathrm{MeV}<E_{\gamma}$. Pair production occurs when photon energies are approximately twice that of the electron mass, and must occur near an electron or nucleus in order to obey conservation of momentum. 


\subsection{Sensor mechanics}

\subsubsection{Particle detection using semiconductors}

In this section certain semiconductor properties will be discussed which are key in understanding the workings of pixel sensors. Figure 2.4 illustrates the effects of ionizing radiation on a semiconducting material. Charged particles travelling through the sensing material deposit energy in the sensor as described in Section 2.1.1, and the absorbed energy is converted into phonons and charge-carrying electron-hole pairs. The movement of the charge-carrying pairs in the sensing material induces a signal pulse on the read-out electronics, which then convert the signal into digital information during the read-out process.

In a bulk semiconductor free of spatial charges, the electric field $E(x)$ is similar to that of a plate capacitor and is given by

$$
E(x)=\frac{V_{b a a s}}{d}
$$

where $V_{\text {bras }}$ is the bias voltage applied to the semiconductor leads, $d$ is the thickness of the material and $x$ is a reference point in the sensor along the direction of the applied bias voltage. The electric field is constant over the bulk of the detector.

Typical silicon sensors utilize the junction of doped p- and n-type silicon to generate semiconductivity. When a $\mathrm{p}-\mathrm{n}$ junction is formed the electrons from the $\mathrm{n}$-type material recombine with the excess holes in the p-type material, and vice versa via a process called charge diffusion. The recombination of the mobile charge carriers leaves behind a stationary spatial charge in the form of ionized atoms, and this creates an electric field opposing the movement of electrons at the junction. The area containing the electric field, which opposes the movement of charge diffusion, is called the depletion region, and in the case of full material depletion the electric field is given 


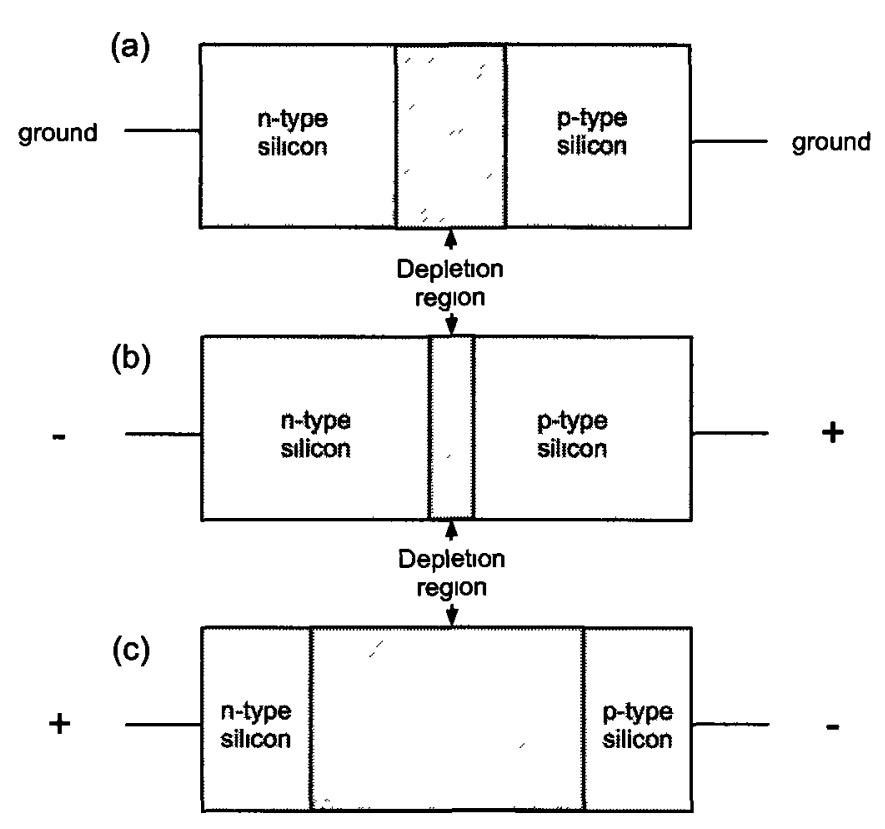

Figure 2.3: The depletion region in a semiconductor can be controlled by applying a bias voltage across the material. (a) shows no applied bias voltage, with a depletion region in full equilibrium. (b) shows a partial forward bias, which shrinks the depletion region pulling excitons towards the opposing electrodes. (c) shows a reverse bias, which increases the width of the depletion region and prevents charge flow across the material.

by

$$
E(x)=\frac{2 V_{b a a s}}{d^{2}} \cdot(x-d) .
$$

Applying a forward bias voltage across the semiconductor can shrink this depleted region, while applying a reverse bias across the semiconductor can cause it to grow to nearly the full width of the detector. In the depleted region it is possible for incident particles to deposit energy via radiation or hard scattering. The effects of a forward and reverse bias on a silicon semiconductor can be seen in Figure 2.3. In the case of a silicon sensor, the width of the depleted region, $W$, is given by 


$$
W=\sqrt{2 \epsilon_{0} \epsilon_{\mathrm{S} 1}\left(V_{\mathrm{b} \text { as }} \mu \rho\right)}
$$

where $\mu$ is the carrier mobility, $\epsilon_{0}$ is the electric permittivity of free space, $\epsilon_{\mathrm{S}}$ is the electric permittivity of silicon and $\rho$ is the resistivity of the material [15]. If the applied bias voltage is not high enough to extend the depleted region across the entire material, neutral regions near the electrodes will exist where no electric field can separate electron-hole pairs generated from energy deposition. Charge-carrier pairs generated in the neutral regions will recombine and result in the loss of signal charge.

Charge builds up on both sides of the junction, which turns the depleted region into an effective capacitor. The charge build-up acts as noise in a semiconducting sensor, and must be reduced to increase hit reconstruction efficiency. The induced capacitance $C$ is given by

$$
C=\frac{\epsilon_{0} \epsilon_{\mathrm{S} 1}}{W}=\sqrt{\epsilon_{0} \epsilon_{\mathrm{S} 1} / 2 V_{\mathrm{blas}}}
$$

Maximizing the bias voltage within the operational limits of the device increases the thickness of the depletion region and minimizes induced capacitance, increasing the signal-to-noise $(\mathrm{S} / \mathrm{N})$ ratio. Sensors with a depleted region which extends the full width of the detector maximize the $\mathrm{S} / \mathrm{N}$ ratio of the detector.

When charge-carrier pairs are produced in the depleted region of the material, the electric field separates them and they propagate towards the electrodes along the electric field lines. Random thermal motion can cause the charge carriers to deviate from the electric field lines. These deviations skew the arrival times of the charge carriers into a Gaussian profile with width

$$
\sigma=\sqrt{2 \frac{k_{b} T}{e} \mu t}
$$




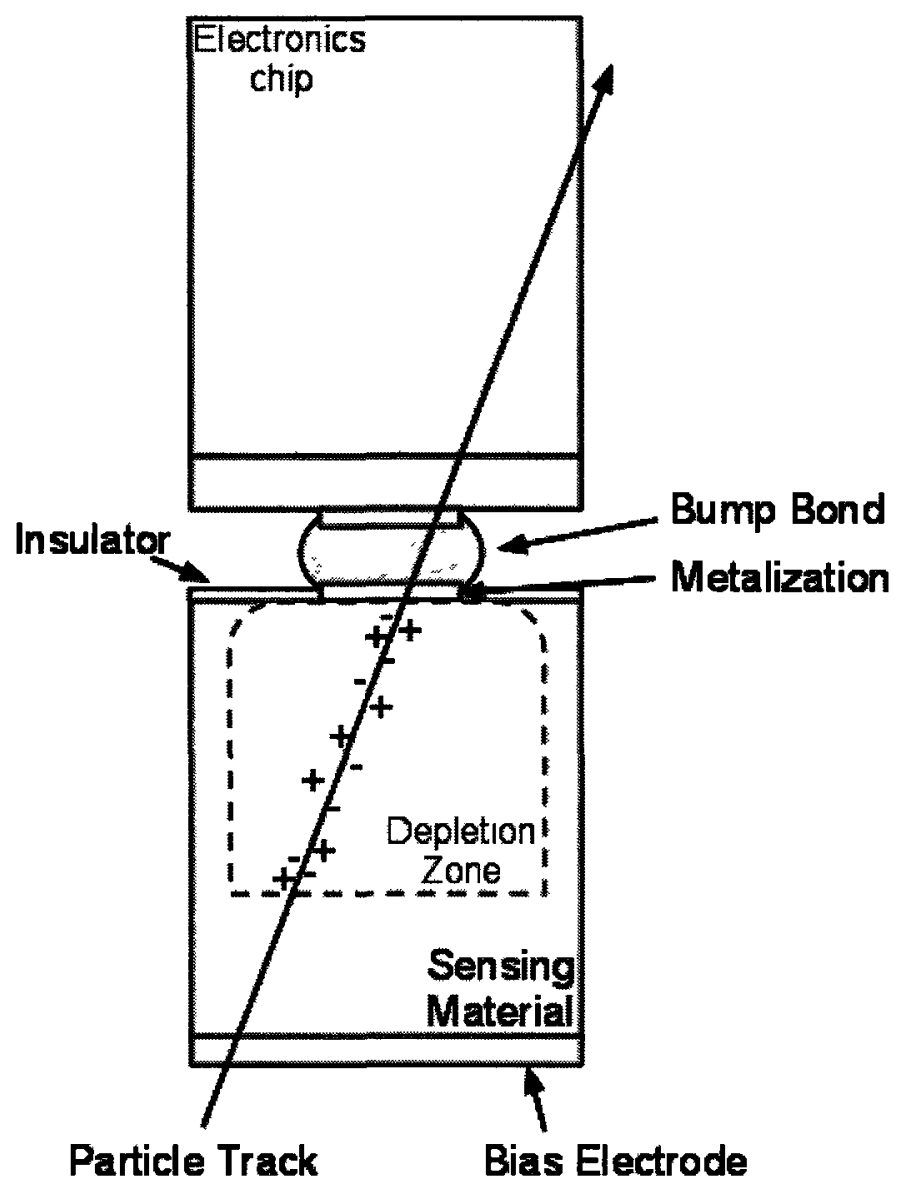

Figure 2.4: Schematic of a single semiconducting pixel. A charged particle passes through the depletion region and injects charge into the sensor which is drained out the read-out electronics. The depletion region depends on the bias voltage applied across the sensor, and the properties of the material used.

where $t$ is the duration of the diffusion. Figure 2.4 illustrates the theory outlined above, showing the result of a charged particle passing through an active semiconductor. The metallization of detectors consists of a gold backplane used to apply the bias voltage directly to the sensor.

Energy deposited in semiconductors can be consumed generating electron-hole pairs, but energy can also be lost through Raman scattering in the crystalline lattice. This second interaction means that the energy required to generate an electron-hole pair is more than the energy required for an electron to jump the bandgap. The 
energy required to generate an electron-hole pair depends on the material used. The properties of different sensors are discussed in Section 2.2.2.

\subsubsection{Diamond as a sensor material}

Several new sensor concepts are being developed to increase the radiation hardness of sensors in preparation for the sLHC. Quantum wells are being produced using p$\mathrm{n}$ junctions in silicon to produce planar and 3D structures, and they are producing promising results, while having the added benefit of an existing infrastructure to build them. The use of junction-based semiconductors as a sensing material is not without issues (see Section 2.3), and so other sensor materials such as diamond are being researched in the hopes that they will eliminate some of those problems.

The material properties of diamond differ greatly from the properties of silicon, and some of the key differences are outlined in Table 2.2. Diamond is roughly $3 / 2$ times denser than silicon, with a thermal conductivity better than that of copper. This makes diamond an excellent conductor to remove heat from the attached readout electronics. The rarity, size and concentration of impurities found in naturally grown diamonds make them useless for the purposes of particle physics, but recent developments in the field of diamond synthesis are making it possible to overcome the natural limitations of the material.

Electrical-grade diamonds are typically synthetic p-type semiconductors doped with low concentrations of boron in the place of carbon atoms. Without the boron impurities, diamond would typically be a natural insulator with a relatively large bandgap of $5.5 \mathrm{eV}$. The intrinsic carrier concentration in diamond is less than $1 \mathrm{~cm}^{-3}$, and the thermally generated leakage current is typically on the order of $10 \mathrm{nA}$ for a $250 \mu \mathrm{m}$ thick sensor. This near-zero charge carrier concentration allows diamonds to be operated at room temperature even after intense irradiation without significant leakage currents. 


\begin{tabular}{lrrcr}
\hline \hline property & symbol & $\mathrm{Si}$ & Diamond & Units \\
\hline atomic number & $\mathrm{Z}$ & 14 & 6 & \\
density & $\rho$ & 2.33 & 3.52 & $\mathrm{~g} / \mathrm{cm}^{3}$ \\
sensor thickness & $\mathrm{d}$ & 250 & & $\mu \mathrm{m}$ \\
\hline bandgap & $E_{g}$ & 1.12 & 5.48 & $\mathrm{eV}$ \\
electron mobility & $\mu_{e}$ & 1450 & 1800 & $\mathrm{~cm}^{2} / \mathrm{Vs}$ \\
hole mobility & $\mu_{h}$ & 505 & 1000 & $\mathrm{~cm}^{2} / \mathrm{Vs}$ \\
mean energy for e/h creation & $E_{e / h}$ & 3.61 & 13.1 & $\mathrm{eV}$ \\
\hline thermal conductivity & & 1.48 & $>1800$ & $\mathrm{Wcm}-1 \mathrm{~K}^{-1}$ \\
resistivity & $\rho$ & $10^{3}$ & $\approx 10^{13}$ & $\mathrm{k} \Omega \mathrm{cm}$ \\
breakdown voltage & $V_{b}$ & 0.3 & 0.5 & $\mathrm{MV} / \mathrm{cm}$ \\
\hline Mean energy loss per MIP & $\frac{d E}{d x}$ & 3.88 & 6.14 & $\mathrm{MeV} / \mathrm{cm}$ \\
& & 108 & 47 & $\mathrm{e} / \mu \mathrm{m}$ \\
radiation length & $X_{0}$ & 9.4 & 12.1 & $\mathrm{~cm}$ \\
\hline \hline
\end{tabular}

Table 2.2: Material properties of Silicon and Diamond [10]. 
The low carrier concentration of diamond means that a reverse biased p-n junction is not necessary for efficiently operating a diamond semiconductor. Diamond sensors also have the added benefit of collecting charge across the entire sensor rather than just a depleted region. Special care must be taken, however, so that the charge injected in the sensor is collected by the readout electronics before the charged particles recombine, or are trapped in various lattice imperfections.

Because diamonds have no pn-junction the sensor is free of space charge in the bulk, which allows for charge collection even at low bias voltages. The higher chargecarrier mobility in diamond also results in lower charge collection times (see Table 2.2). An ideal diamond sensor $200 \mu \mathrm{m}$ thick would result in an electron collection time of $1.8 \mathrm{~ns}$ and would scale linearly with the applied bias voltage. Holes would be collected in roughly the same amount of time.

Because diamond is not as widely used in particle physics as silicon, it is necessary to derive the energy deposition and electron-hole generation energy by scaling the values known for graphite by the mass-density ratio of diamond to graphite. Using this method we obtain $d E / d x=6.14 \mathrm{MeV} / \mathrm{cm}$, and we can obtain $E_{\mathrm{e} / \mathrm{h}}=13.1 \mathrm{eV}$ by calculating how much energy is consumed by the band-gap jump, and Raman scattering in electron-hole pair production. Based on these values, we expect a 200 $\mu \mathrm{m}$ diamond to observe approximately $9400 \mathrm{e}^{1}$.

\subsubsection{Production of synthetic diamonds}

Synthetic diamonds wafers as seen in Figure ?? are created by way of a process called chemical vapor deposition (CVD). Diamonds are grown by depositing the carbon from a microwave plasma onto a substrate, while suppressing the formation of graphite layers using selective etching techniques. Diamonds can be grown directly onto bias plates which removes the need for implantation of electrodes onto the sensor.

\footnotetext{
${ }^{1}$ Electron charges
} 
Two types of crystalline lattices may be formed in a CVD process: poly-crystalline diamond (pCVD) and single crystal diamond (scCVD).

pCVD diamond arrises when multiple seed crystals form early in the CVD process. The growth process will eventually become dominated by a smaller fraction of the seeds creating a grain-like structure. The substrate side of pCVD diamond is of low quality compared to the growth side with many grain boundaries, and so it is typically ground down to improve sensor performance. The growth side may also be ground down to produce a smooth surface if the application requires it. The grain lattice structure of pCVD diamonds also introduces a polarization effect on top of the existing electric field resulting in systematic, position-dependent variations in the spatial reconstruction of charged tracks as seen in [16].

scCVD diamonds are grown in a similar fashion, with the exception that an appropriate single crystal substrate must be used as a seed for the growth. Current generation scCVD diamonds are limited in size to approximately $1 \mathrm{~cm}^{2}$ [17]. In both cases, the surface side of the diamonds are typically very granular and must be ground and polished before the diamonds are able to be used in sensor applications. The grinding and polishing of the sensor is also used to obtain the desired sensor thickness.

Charged particles will deposit energy in the sensor, generating electron-hole pairs which travel along the electric field lines to be collected at the pixel electrodes. Crystal granularities will affect the movement of charge in the sensor, potentially trapping charge-carriers and decreasing the efficiency of charge reconstruction. Growing pCVD diamonds longer allows the crystal to be grown with a much larger grain size, but can be very expensive. The decreasing granularity of pCVD wafers from the substrate side (bias electrode end) to the growth side (pixel electrode end), as well as a comparison of the crystalline structures of pCVD (on the left) and scCVD (on the right) can be seen in Figure 2.5 . 


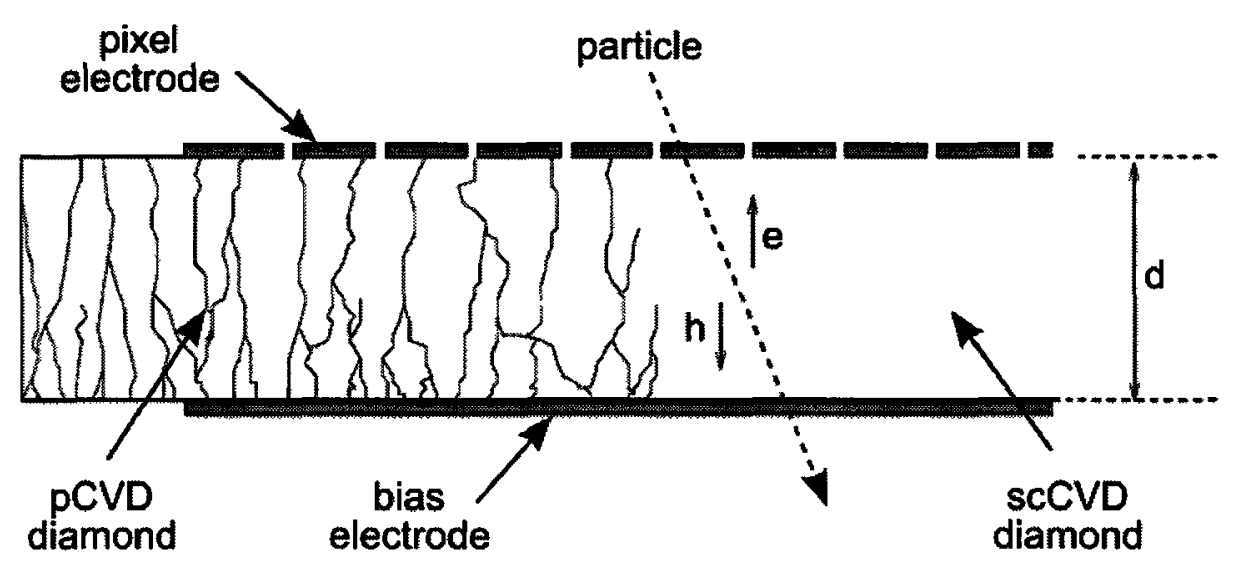

Figure 2.5: Example of metallized pCVD and scCVD diamond sensor setup. Injected charge from tracks are affected by the concentration of impurity and lattice deformations in the sensor, making single crystal diamonds a superior sensor material [18]. Used with permission.

The parameters for energy deposition in diamond have not been explicitly derived. The energy deposited can be obtained by scaling the energy deposition value of graphite by the ratio of densities of graphite to diamond. This method yields approximately $6.14 \mathrm{MeV} / \mathrm{cm}$, and the energy required to generate an electron-hole pair is found to be $13.6 \mathrm{eV} /$ pair. Using these values we expect the mean charge deposition to be 9400 e in a $200 \mu \mathrm{m}$ sensor, and approximately 19000 e in a $395 \mu \mathrm{m}$ sensor.

As described in Section 2.1.1 it is often more meaningful to express energy loss in terms of most probable charge deposited. In order to estimate these parameters, we scale thickness of the detector by the ratio of the densities of silicon and diamond, and use the equation 2.4. Using this method, $\Delta_{p}=13.4$ ke is obtained for a $395 \mu \mathrm{m}$ sensor, and $\Delta_{p}=6500 \mathrm{e}$ for a $200 \mu \mathrm{m}$ sensor. The ratio of MPV to mean is therefore 0.72 for a $395 \mu \mathrm{m}$ thick diamond, and decreases to 0.69 for a $200 \mu \mathrm{m}$ sensor. Because the ratio is highly sensitive to sensor thickness, it must be calculated explicitly for each sensor.

Diamonds are currently used in the ATLAS detector as a beam condition monitor (BCM) due to their high radiation tolerance, and are expected to receive doses on 


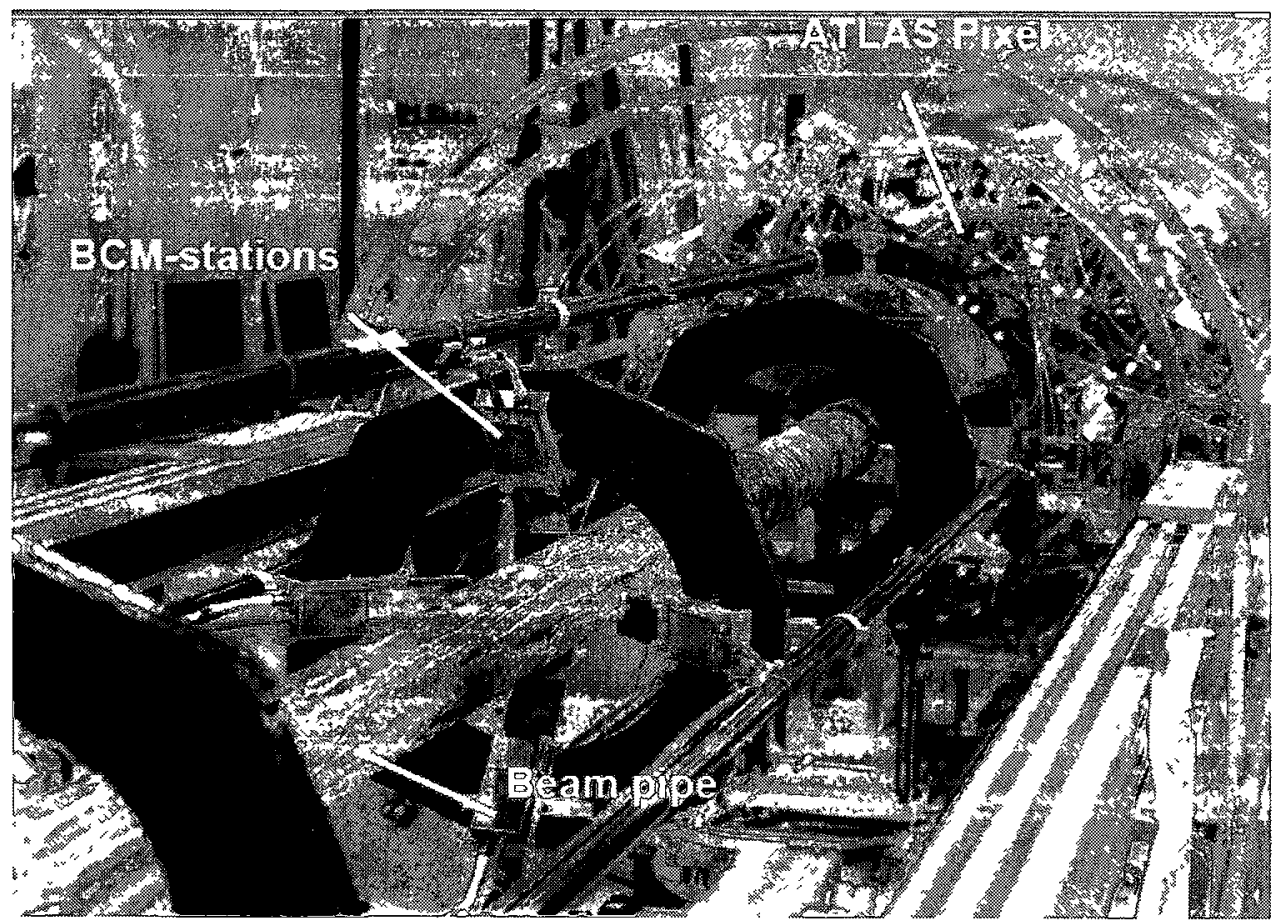

Figure 2.6: Photo of the ATLAS beam condition monitor used to determine in real time if particles are straying from their desired trajectory, and invoke beam dumps to prevent damage to the detector [20]. Used with permission.

the order of $10^{16}$ particles $/ \mathrm{cm}^{2}$ - a dose typical solid state detectors could not survive. The setup of such a $\mathrm{BCM}$ is shown in Figure 2.6; it is placed along the beam-pipe down and upstream of the interaction point. Diamonds are currently being studied for use in various applications such as calorimetry and position reconstruction. Other collaborations which have implemented a diamond $B C M$ are $C D F, B A B A R$, Belle, and $\mathbb{C M S}[19]$.

\subsection{Radlation damage}

Because pixel detectors are best used in regions near the interaction point of experiments, they are exposed to high levels of radiation. This large flux of particles is responsible for considerable damage to the sensing material and read-out electronics. Damage of this kind is divided into surface and bulk defects. Surface defects include 
effects on the covering dielectrics and interface region of the sensor, such as ionization in the silicon oxide connecting the sensor to the dielectric materials. Surface damage has no direct consequence on the operation of particle sensors beyond its consideration in the design of the detector to account for its effect on the electric field.

The main concern in the design of pixel detectors is the effects of bulk defects in the sensor. Semiconductors have a crystalline lattice structure, which makes them vulnerable to radiation damage. High energy particles can scatter off of nuclei in the material introducing crystal imperfections. The minimum recoil energy required to bump an atom out of the lattice structure is approximately $25 \mathrm{eV}$. Electrons, being relatively light particles, require nearly $260 \mathrm{eV}$ of kinetic energy to cause such a defect in silicon, while larger particles such as protons and neutrons only require $190 \mathrm{eV}$. If a recoiling atom has enough energy it can cause further defects in the lattice, causing a cascade of localized dislocations.

The concentration of crystal defects will increase over years of operation to the point where they will exceed the initial doping concentrations of the sensor, and the material properties will then largely be determined by the defects. Bulk damage brings about several secondary effects visible in Figure 2.7; these are a result of energy levels appearing between the valence and conduction bands from atoms dislodged from the crystalline lattice.

Different particles of different energies produce different levels of damage in a reference material as demonstrated in Figure 2.8. In order to compare radiation damage across all particle and energy types, it is necessary to scale the damage with a quantity called nonionizing energy loss (or NIEL). This quantity parametrizes the energy deposited in the sensor which wasn't used in reversible ionization processes. 1 $\mathrm{MeV}$ neutrons are typically used as the reference particle with fluence $\Phi_{e q}$. Physical fluences $\Phi_{\text {phys }}$ can be converted to a neutron equivalent $\Phi_{e q}$ using [21]. While some experiments are showing that NIEL scaling is not fully established [22], it serves as 


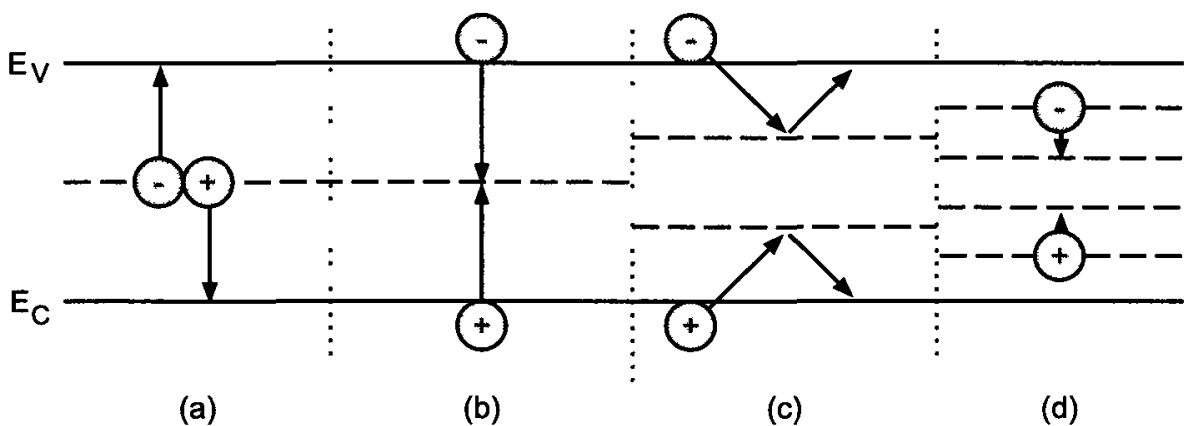

Figure 2.7: Atoms dislodged from the crystalline lattice introduce energy levels between the valence $\left(E_{V}\right)$ and conduction $\left(E_{C}\right)$ bands (dashed lines) of the material and can assist in (a) thermally generated electron-hole pairs, (b) assisted electron-hole pair recombination, (c) temporary charge trapping and (d) effective doping.

an excellent tool for comparing relative effects since measuring the damage effects for all particles of all energies is very impractical.

\subsubsection{Increased leakage current}

The lifetime of charge carriers $(\tau)$ and the leakage current $\left(I_{d}\right)$ are affected by the radiation damage produced at intermediate energy levels. The decrease in the lifetime of charge carriers is given by

$$
\frac{1}{\tau}=\frac{1}{\tau_{0}}+k_{r} \Phi,
$$

where $\tau_{0}$ is the initial charge carrier lifetime before irradiation, $\Phi$ is the exposed particle fluence of the detector, and $k_{r}$ is the lifetime damage rate, a material and particle dependent constant which parametrizes the rate of sensor depletion. The change in leakage current for a fully depleted sensor is given by

$$
I_{d}=I_{0}+\alpha \cdot \Phi \cdot A \cdot d,
$$

where $I_{0}$ is the initial leakage current of the sensor before irradiation, $\alpha$ is a damage 


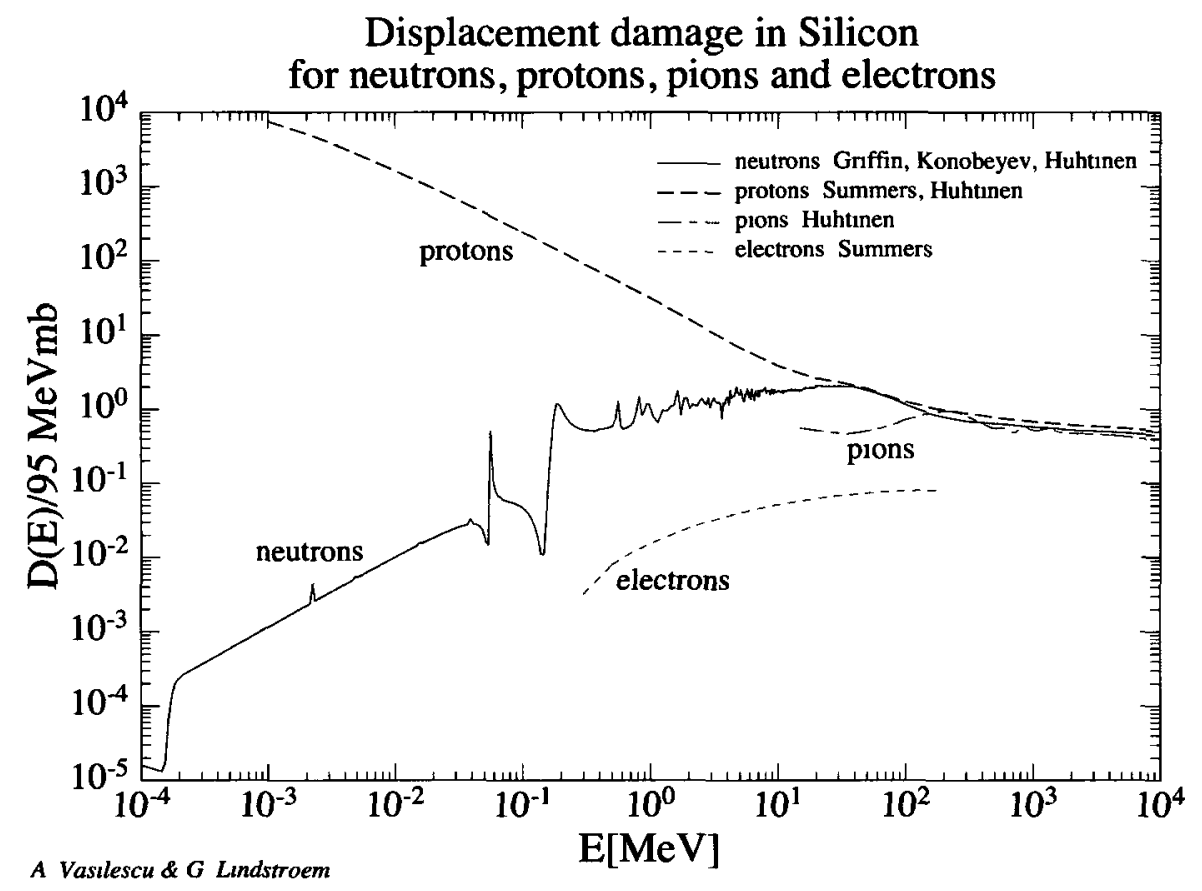

Figure 2.8: Displacement damage for neutrons, protons, pions and electrons as a function of particle energy scaled to the damage of $1 \mathrm{MeV}$ neutrons [23]. Used with permission. 
coefficient similar to $\beta, A$ is the cross-sectional area of the detector, and $d$ is the width of the detector. A value of $\alpha=3.99 \pm 0.03 \times 10^{-17} \mathrm{~A} / \mathrm{cm}$ has been found to be in agreement with various types of silicon detectors [24,25]. Diamond detectors have been shown to exhibit no increase in leakage current after irradiation [26]. Because the leakage current also depends on the temperature of the sensor, irradiation of the sensor can lead to a runaway increase in temperature, and therefore leakage current. This increase in leakage current degrades the maximum achievable $\mathrm{S} / \mathrm{N}$ ratio, and can ultimately mean the destruction of the device.

\subsubsection{Decreased charge collection distance}

Defects in the crystalline lattice introduce energy levels near the conduction and valence bands as seen in Figure 2.7. These energy levels act as recombination and production centers as well as trapping sites, preventing the flow of charge in the sensor. Trapped charge can either recombine or be knocked loose by thermal activity. The trapping of charge can be exploited in a process commonly referred to as pumping.

Pumping a sensor is achieved by exposing it to an excess of $\beta$ radiation. The electrons passing through the sensor will fill the traps, increasing the charge collected at the read-out electrodes. Sensors which have not been exposed to $\beta$ radiation before use will show an increase in signal charge as exposure increases to a limit, when charge is ejected from traps at a rate equal to that of capture.

Trapping centers are common in all semiconducting materials, and are particularly prevalent in sensors with imperfect lattice structures such as pCVD diamond, or composite materials. When assigning received signal charge to a particle which transitioned in the detector we must only consider signal observed in a specific interval, eliminating contributions from charge trapped in lattice imperfections. Sites with trapping times long enough to hold charge beyond the allowed travelling time 
of signal carriers, but short enough trapping times that they don't contribute to signal charge from another particle transition, limit the charge collection properties of materials.

The carrier lifetime ( $\tau$ from equation 2.12) is used to characterized sensor damage, but much more useful is the charge collection distance (ccd), given by

$$
\operatorname{ccd}=\tau \cdot v_{c}=\tau \mu E
$$

where $v_{c}$ is the drift velocity, or travel speed $\mu E$, of the charge carrier. The ccd is the average distance a charge carrier will travel in the sensor before recombining or being captured by charge traps. This side-effect of radiation damage affects charge carriers differently, and thus must be characterized accordingly. $k_{r}$ from equation 2.12 has been observed to be $k_{r}=(5.4 \pm 0.4) \times 10^{-16} \mathrm{~cm}^{2} / \mathrm{ns}$ for electrons, and $k_{r}=(6.6 \pm 0.9) \times 10^{-16} \mathrm{~cm}^{2} / \mathrm{ns}$ for holes in silicon independent of the type of doping [25].

Damage coefficients for both scCVD and pCVD diamond have been observed to be less than silicon, though on the same order of magnitude [18]. Impressively, the damage coefficient for scCVD and pCVD diamonds appear to be the same $\left(k_{r} \approx\right.$ $\left.2 \times 10^{-16} \mathrm{~cm}^{2} / n s\right)[27,28]$, suggesting that the purity of the lattice structure does not increase the radiation hardness of the sensor. Charge carriers produced further away than the ccd of the material are not likely to be observed, and so no significant signal amplitude is observed in sensors with a thickness larger than their ccd. This effect is visible in Figure 2.9.

The introduction of energy levels in the bandgap of the sensor also affects the effective doping concentration in the semiconductor as seen in Figure 2.7 (d). This effect particularly changes the properties of junction based semiconductors, resulting in a reduction in the number of donor levels and an increase in the number of acceptor 


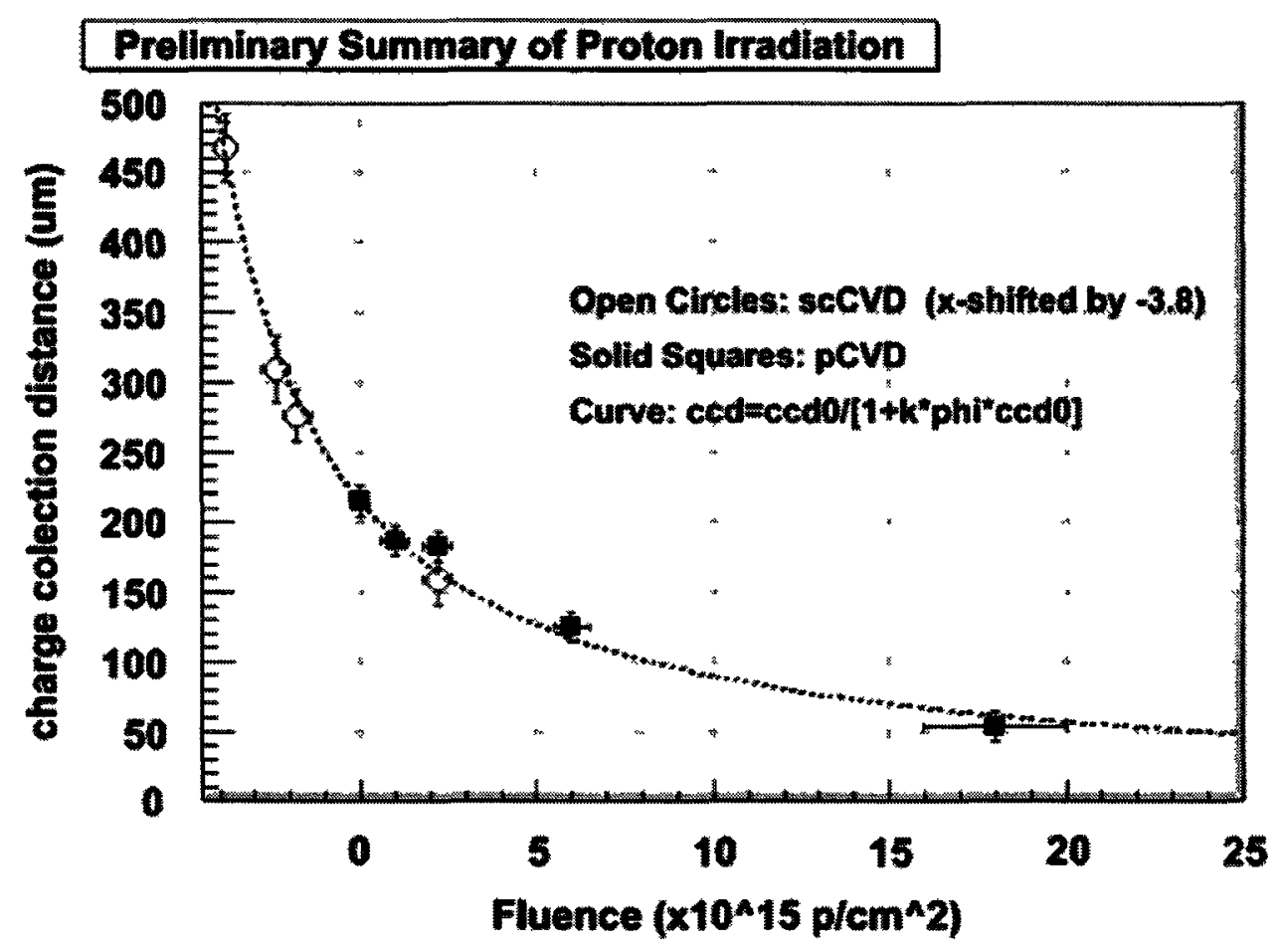

Figure 2.9: Summary of proton irradiation results for pCVD (solid squares) and scCVD (open circles) diamond up to a fluence of $1.8 \times 10^{13} 24 \mathrm{GeV}$ protons $/ \mathrm{cm}^{2}$. The black curve is a standard damage curve $\left(1 / c c d=1 / c c d_{0}+k \phi\right)$ scCVD irradiation has been shifted to the left by a fluence of $3.8 \times 10^{15}$ protons $/ \mathrm{cm}^{2}$, where it's unirradiated charge collection distance falls on the curve. With this shift the pCVD and scCVD data fall on a single curve indicating a common damage constant for both materials [28]. Used with permission. 
states. Doping concentrations can be dropped to the point where a type inversion occurs, and n-type materials will begin behaving like p-type materials, and vice versa. This effect requires the presence of a junction in order to take effect, so sensors such as diamond are immune to this type of damage.

Consult [15] for a more complete treatment of radiation damage in semiconducting sensors. 


\section{Chapter 3}

\section{ATLAS pixel modules}

The ATLAS pixel read-out electronics have been designed with a radiation tolerant layout and several redundancies. These measures have demonstrated a radiation tolerance of up to 10 Mrads (or $2 \times 10^{14}$ protons $/ \mathrm{cm}^{2}$ ) [29]. $24 \mathrm{GeV}$ proton irradiation studies are currently being performed on all sensors, but there is some debate as to the equivalence of proton vs neutron irradiation and proton vs pion irradiation (as discussed in Section 2.3). Irradiation tests with $300 \mathrm{MeV} / \mathrm{c}$ pions and $1 \mathrm{MeV}$ neutrons exist, and some agreement has been shown regarding the characterized damage of CVD diamonds [30].

The irradiation of ATLAS pixel devices are handled at CERN's PS facilities which are capable of irradiating devices using $24 \mathrm{GeV} / \mathrm{c}$ protons. The PS irradiation facilities are also capable of fast and slow neutron irradiation and mixed hadron fields. New sensor materials are being tested at fluences higher than the radiation tolerance of $\mathrm{FE}$ I3, and the read-out electronics need to be replaced after irradiation. All irradiations were carried out at room temperature with sensors in an unbiased state.

ATLAS Pixel modules are fully assembled read-out chips bump bonded to sensor material. There are two module types: full modules are intended for use in the ATLAS detector, and single-chip modules are intended for testing purposes. Both modules use FE-I3 chips as their read-out system, and are used by all ATLAS R\&D 


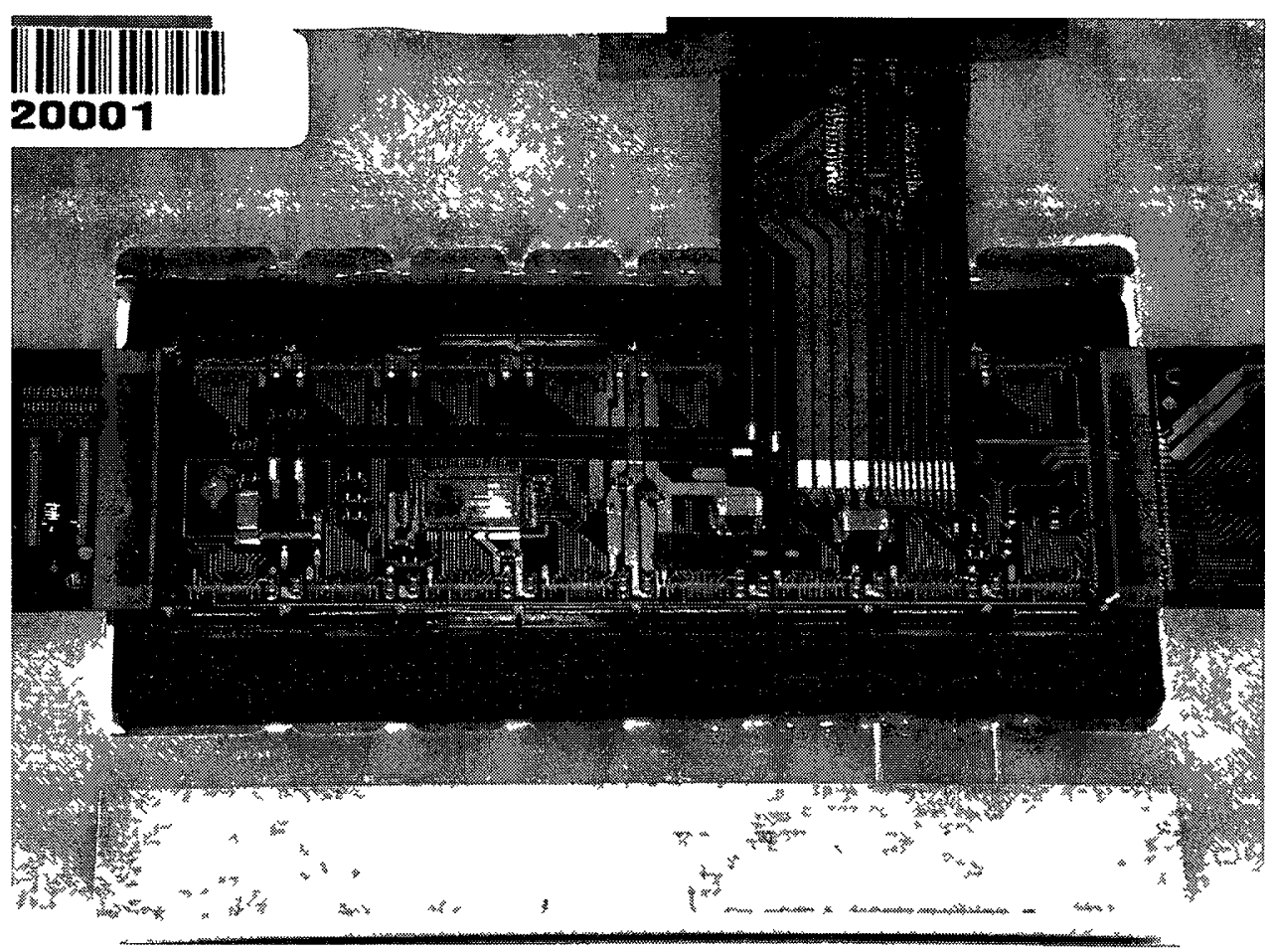

Figure 3.1: Photo of a full ATLAS pixel module with flex-hybrid. The sensing material cannot be seen in this photo

groups at CERN for the testıng of new sensing materials as pixel detectors.

Full modules combine 16 single-chip assemblies in an $8 \times 2$ layout bump bonded to the sensor material, and are connected using a flex-hybrid which distributes power and provides an interface to the read-out and control system. The lower portion of Figure 3.2 shows a top-down view of a full module, labelling key parts which will be discussed below.

\subsection{Current generation sensing material}

A $250 \mu \mathrm{m}$ thick n-type bulk silicon sensor is used with a $\mathrm{p}+$ type back plane. Individual pixels are made with $n+$ implants creating an $n-i n-n$ design, specifically for the collection of electrons on the read-out electronics. The bulk sensor depletes at $60 \mathrm{~V}$ when unirradiated, and the silicon has been oxygenated to increase radiation 


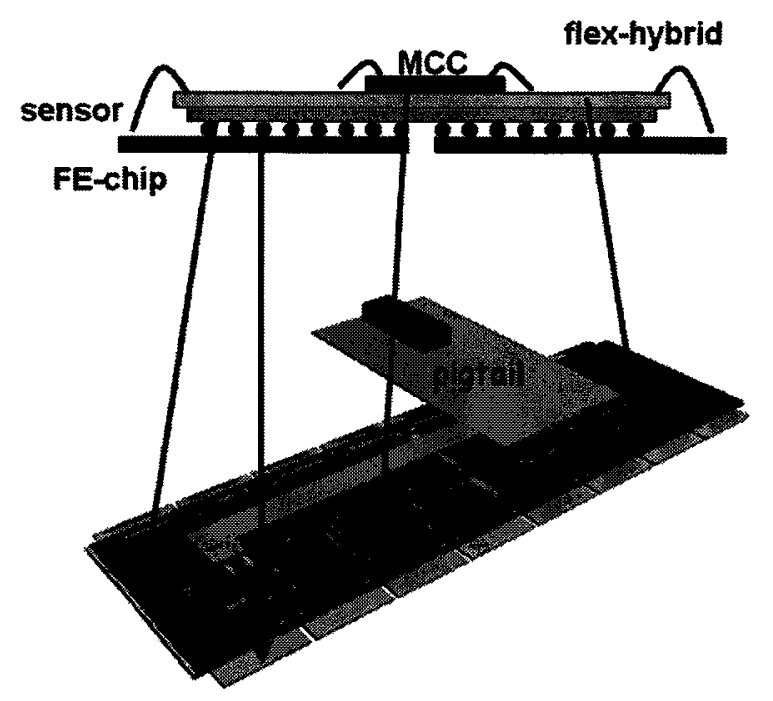

Figure 3.2: Simplified schematics of a full ATLAS pixel module displaying the location of the front-end electronics chips, the pigtail read-out connector and the module control chip (MCC) [18]. Used with permission.

tolerance. The introduction of a known impurity, such as oxygen, into silicon getters the primary defects of induced by irradiation by removing vacancies in silicon $[31,32]$. The sensor has an active area of $1.64 \times 6.08 \mathrm{~cm}^{2}$ and is surrounded by a $500 \mu \mathrm{m}$ guard ring to prevent bias voltage breakdown. This is especially needed when irradiation requires an increase of the applied bias voltage to maintain functionality.

The sensor has a total of 47232 read-out channels across all 16 chips in $400 \times 50$ $\mu \mathrm{m}^{2}$ pixels, but in order to cover the dead space between chips on the board, pixels near the edges of chips are ganged with pixels between chips. Pixels in the dead space are also enlarged to $600 \times 50 \mu \mathrm{m}^{2}$ (see Figure 3.3 for illustration). 


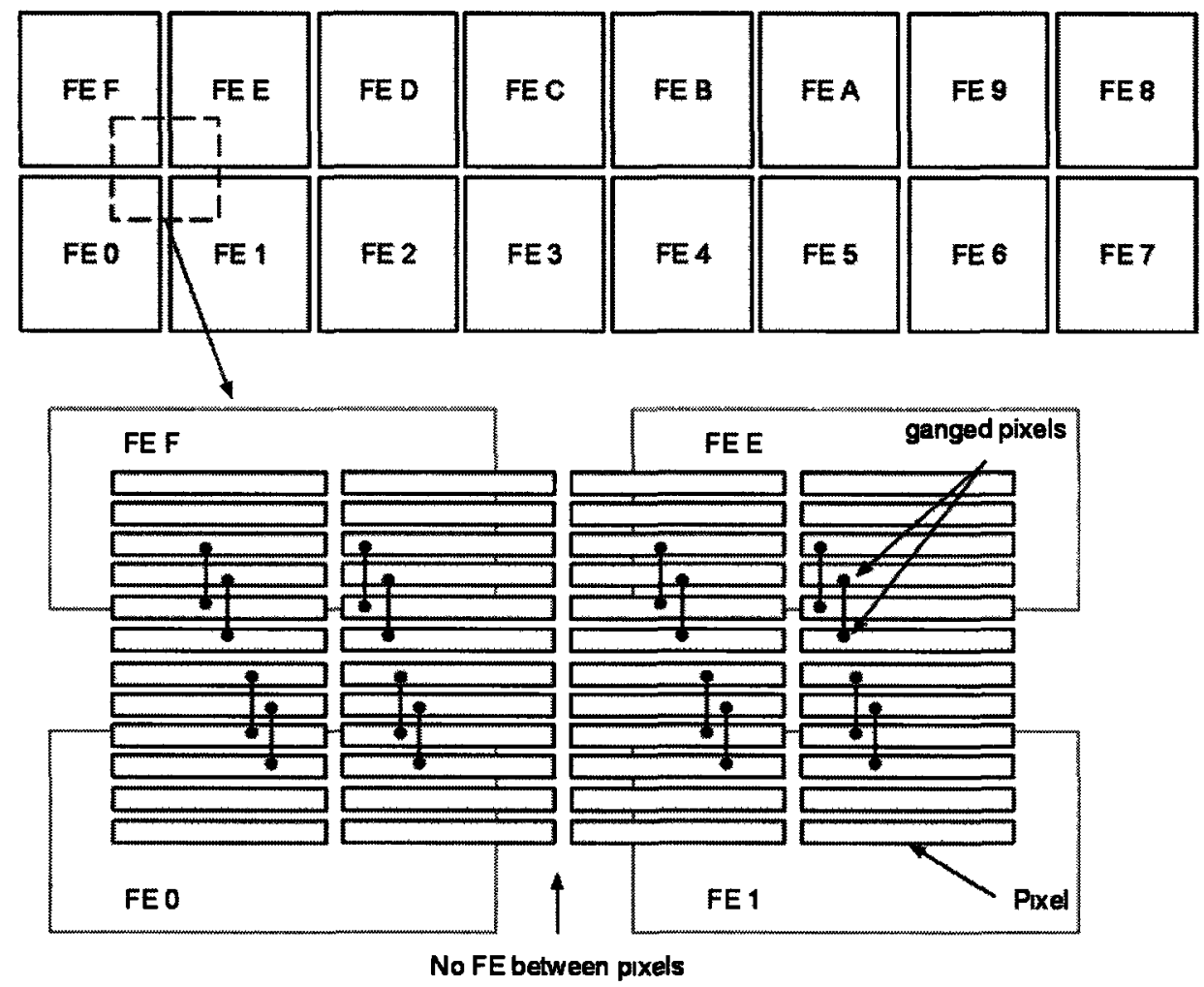

Figure 3.3: Illustration of ganged pixels on an ATLAS full module board. The 16 front end chips on an ATLAS full module are assigned a hex ID (0-F). Ganged pixels allow for a complete coverage of a full module's active area, including regions between front-end chips. Pixels are laid out such that the long side of a pixel is in line with the long side of the board. 


\section{$3.2 \quad$ Read-out electronics}

\subsubsection{FE-I3 read-out electronics}

The current ATLAS pixel read-out electronics standard FE-I3 ${ }^{1}$ was developed by the ATLAS collaboration for use in the ATLAS tracking system. The electronics were developed independent of sensing material, and are used by all sensor R\&D groups as a standard pixel read-out system. Using this system allows researchers to benefit from the existing ATLAS framework for sensor calibration and operation, and will make for easy integration into the ATLAS detector when a suitable candidate is selected for the upgrade. The FE-I3 chipset is described in greater detall in [29].

FE-I3 chips have 2880 channels organized in 160 rows $\times 18$ columns, producing an active area of roughly $0.576 \mathrm{~cm}^{2}$. Sensing materials are bump bonded to the readout electronics using $\mathrm{PbSn}$ solder bumps, and gold bias electrodes are are applied to the top and bottom of the sensing material. Each pixel is equipped with a charge sensitive amplifier, a discriminator, and a digital output interface.

On-board memory registers enable the performance of the read-out electronics to be tailored to the user's requirements, and optimally integrate into any pre-existing system.

There are two types of on-board circuits on an ATLAS pixel module: analog and digital circuits. Every channel of the read-out electronics has its own digital and analog blocks, and some global circuits exist for tuning and operational purposes.

The analog block of each channel amplifies signal charge using a charge sensitive amplifier, and compares that charge against a programmable threshold using a discriminator. The pulse generated from this circuit is analyzed by the digital circuitry for read-out and hit reconstruction. The schematics for the analog block of a pixel are shown in Figure 3.4.

\footnotetext{
${ }^{1}$ Front End Iteration 3
} 


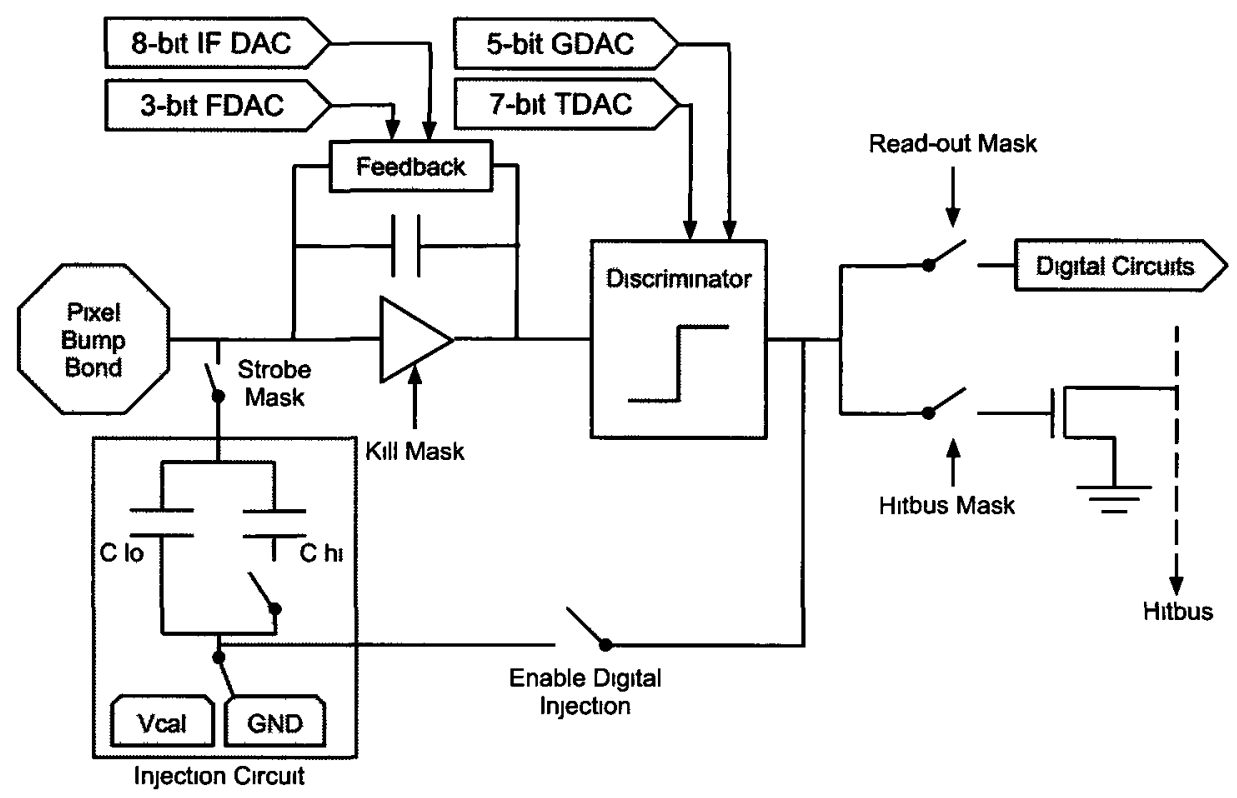

Figure 3.4: Analog circuit schematics of an FE-I3 pixel. The circuit displays the charge injection circuit used for device calibration, the charge sensitive amplifier and discriminator used for signal digitization, and the on-board registers (DACs discussed in Chapter 4) which control the parameters of signal digitization. Several on-board switches exist such as the digital injection, and read-out mask which allow the user to activate and deactivate selected chip functions at will. 
The digital block of each channel digitally timestamps hit information with a BCID, and transmits a hit pixel's address, the BCID, and digitized pulse amplitude information (i.e. the Time over Threshold, discussed in Section 3.2.2) to hit buffers outside the chip's active area. Hits in the buffer are monitored according to their age and are read-out if they are matched up with a received trigger. Hits are deleted from the buffer if their age exceeds a pre-defined trigger latency, and they are not matched up with a received trigger.

Read-out of the chip is completed in two stages: hits are read out into the End of Column (EoC) buffers located at the bottom of the chip, and the EoC buffers are cleared in the second stage where trigger latency is accounted for. EoC buffers are shared between two pixel columns (for a total of 320 pixels per buffer), and can contain a maximum of 64 hits per buffer. While hits are being transferred to the EoC buffers, the pixel is incapable of receiving a second hit. Hits which are sent to full EoC buffers are also lost. These two scenarios mean that the maximum number of hits detectable both in parallel and in series are limited by the electronics.

EoC buffers are cleared in the second stage, and a latency-DAC ${ }^{2}$ is subtracted from the BCID timestamp to match up received triggers to measured hits. Hits matched with received triggers are marked for read-out, serialized and are read-out in turn at the $40 \mathrm{MHz}$ system clock speed. If the time required for the trigger latency has passed, and no triggers are matched up with hits in a buffer, those hits are discarded. An unfortunate consequence of this read-out method is that if a hit takes longer to read out in stage one than the trigger latency, then those hits will be lost since they won't yet be available to stage two for trigger match-up.

\footnotetext{
${ }^{2}$ Digital to Analog Converter
} 


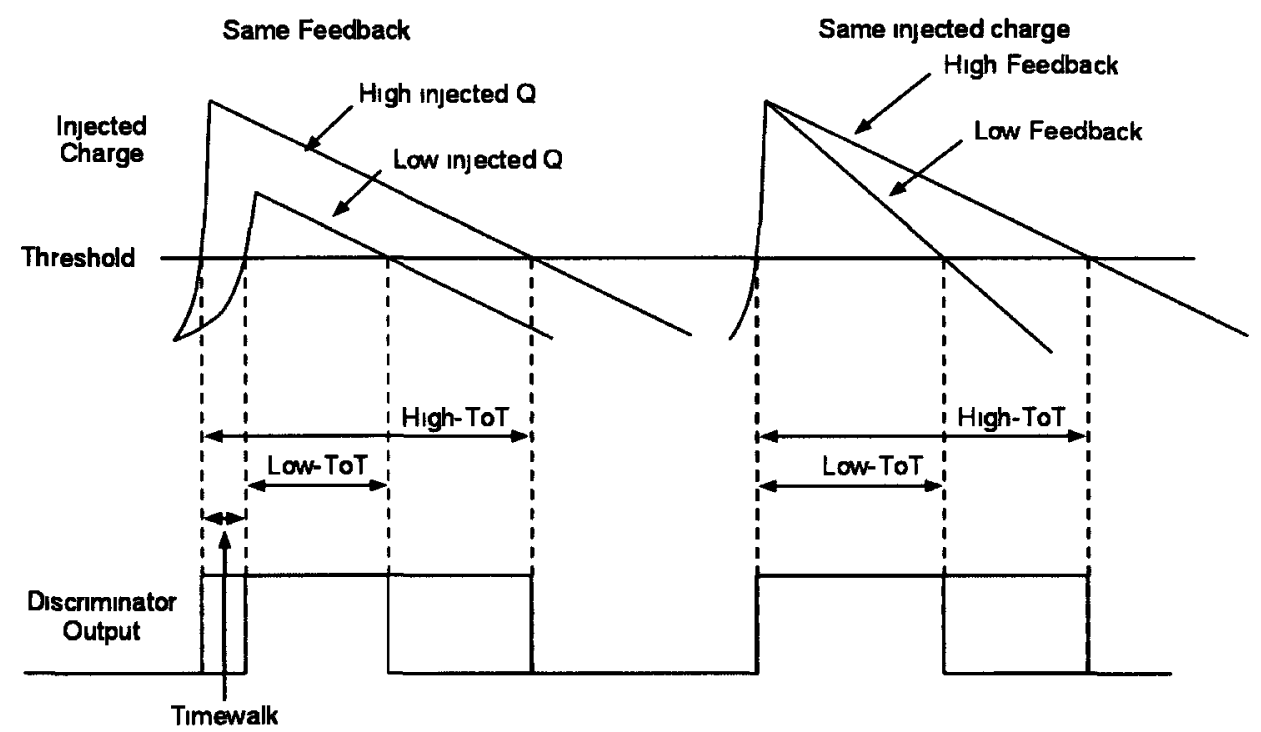

Figure 3.5: Graphical depiction of a time over threshold (ToT) determination. The Figure on the left shows two amounts of charge injected into the electronics. The green being the higher charge deposited crosses the threshold earlier than the lower charge injection in red, causing an effect known as timewalk. The image on the right shows what occurs to a single amount of charge injected with different feedback currents applied to the charge sensitive amplifier. The higher current (depicted in green) takes longer to drain than the lower (in red), resulting in an effective amplification of the observed charge [33].

\subsubsection{Digitizing hit information}

The ATLAS pixel read-out electronics measure several properties of the hit which assist in hit reconstruction and identification. Hit locations are measured using the pixel row and column ID, which gives a position resolution of $\sigma_{\text {short }}=14 \mu \mathrm{m}$ and $\sigma_{\text {long }}=115 \mu \mathrm{m}$ without considering hit information from multiple channels. A 4-bit level $1 \mathrm{BCID}^{3}$ counter measures the amount of time passed between trigger received and hit observed in the detector.

A digital measurement of charge injected into the sensor is emulated using the ToT $^{4}$ method. When signal charge is injected into the sensor material the charge

\footnotetext{
${ }^{3}$ Bunch Crossing Identifier

${ }^{4}$ Time over threshold
} 
induces a voltage in the analog circuit, producing a pulse which is amplified using a charge sensitive amplifier. The design of this amplifier ensures that the pulse peaks at the same time regardless of charge injected into the sensor. This effect means that low charge pulses can reach the discriminator threshold a full $\mathrm{BC}$ count later than larger injected charge pulses. This effect is known as tıme walk is due to the $40 \mathrm{MHz}$ sampling rate of the discriminator, and can be seen in Figure 3.5.

The amplified pulse is then passed through the discriminator. When a pulse is observed from the discriminator, digital circuits measure how long the threshold registers as on, and return an 8-bit bunch crossing count of the pulse length. The charge scaling from the amplifier and discriminator thresholds are known through calibration. This allows us to directly correlate the charge deposited in the sensor to a ToT count. The details of this relation and the calibrations which make this possible are explained in Section 4.3.

\subsection{The flex-hybrid}

The flex-hybrid provides the routing of power, bias voltage and data lines from the ribbon pigtail cable connections to the FE-I3 chipset. It also contains a module control chip (MCC) capable of merging the read-out information from all 16 chips into a single data stream, and distributes control signals from the pigtail to the individual chips. The flex hybrid is made of a $50 \mu \mathrm{m}$ polyamide substrate with copper traces of $25 \mu \mathrm{m}$ thickness on both sides, and is covered in a commercial solder mask material.

In the ATLAS detector, data lines are directly soldered to the flex for pixel modules on the end-cap. Pixel modules in the barrel are connected to the data lines using a connector located on the pig-tail. The kapton material is capable of housing the high voltage traces $(\mathcal{O}(0.5-1 \mathrm{kV}))$ for sensor bias and is radiation hard and will survive the radiation dose received during operation. 


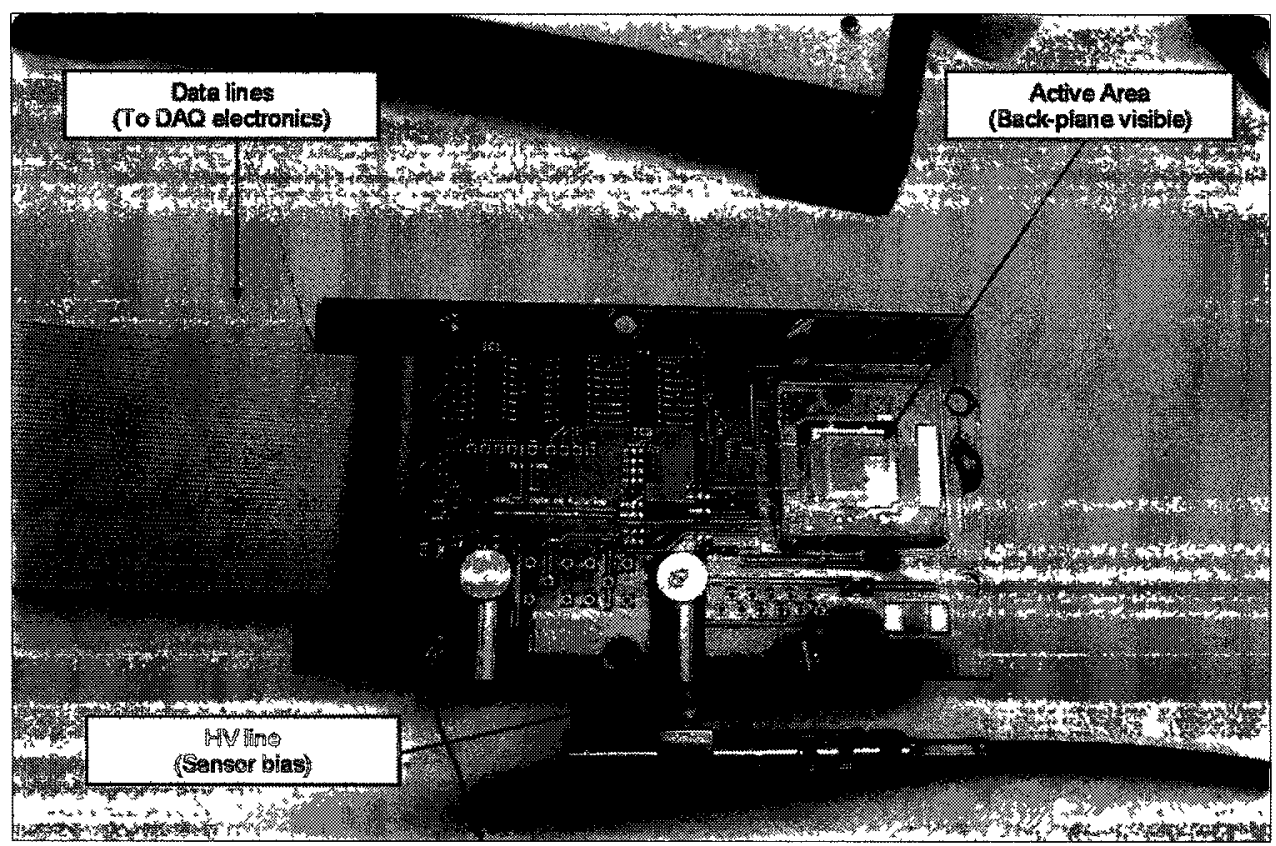

Figgure 3.B: Photograph of the ATLAS pixel detector single-chip module.

Single-chip pixel modules as seen in Figure 3.6 exist for the purpose of lab characterization and testing of sensors which have been adapted to fit on the a single front-end chip. The flex-hybrid is then replaced with a printed circuit board, to which the single-chip assembly is glued. The MCC is discarded from this setup because the device only uses one front-end chip. 


\section{Chapter 4}

\section{Characterization and Calibration of ATLAS Pixel Devices}

I was placed in charge of assembling and calibrating a test stand capable of calibrating ATLAS FE-I3 devices at Carleton University. During the 6 months I determined that a copy of the existing calibration set up at CERN would be impractical, and took the initiative in the integration of a USB based calibration system. I was responsible for determining the hardware required, and installing all the necessary components.

Several issues with the board delayed the completion of the project by several months, but they were eventually overcome with the assistance of the developers. I then produced a working calibration for a supplied FE-I3 chip, trained a group of people on the use of the device. I performed a test of the device using a Sr-90 source, and analyzed the data using software provided by the software developers. The results shown in this chapter are the result of months of debugging, correspondance and trial and error. 


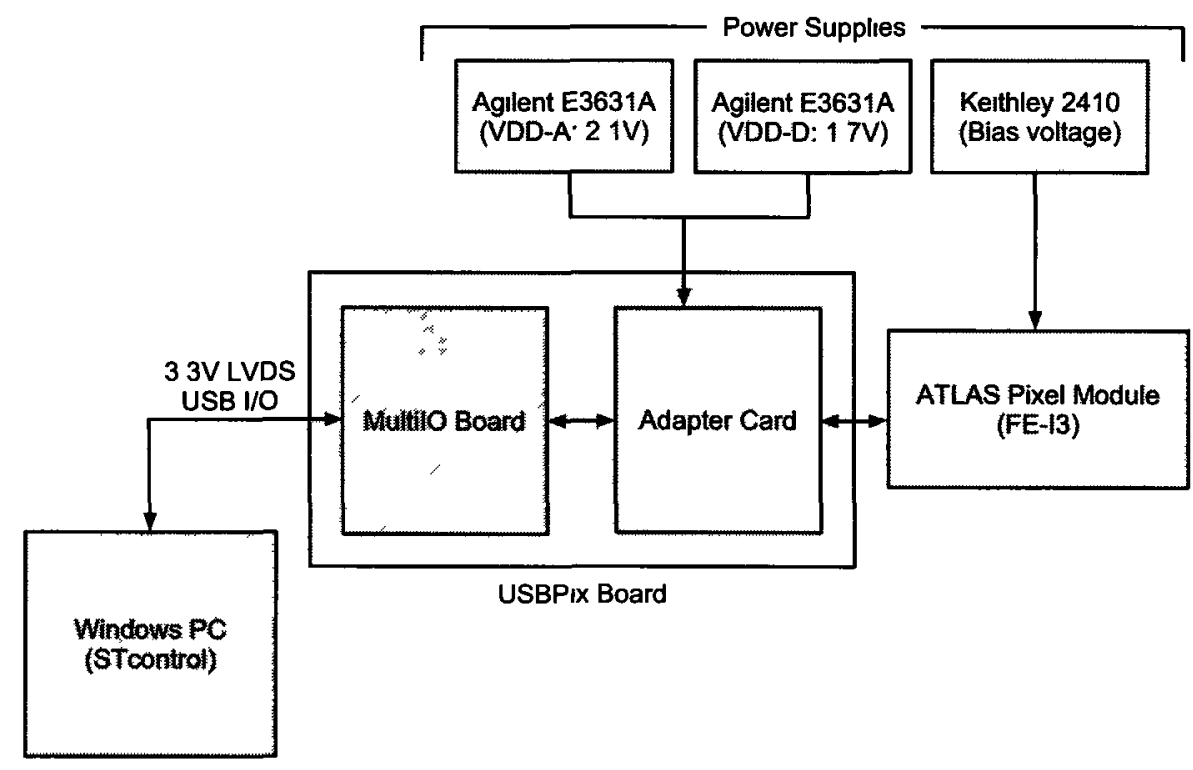

Figure 4.1: USBPix test-stand setup. USBPix Test-stands are used to calibrate and communicate with ATLAS FE-I3 and FE-I4 pixel modules during sensor characterization and eventually during beam tests. The USBPix board consists of two circuit boards: the adapter card is responsible for direct communication with all attached ATLAS pixel modules as well as supplying power to the on-board analog and digital circuits of the FE chips. The MultilO board is responsible for communication between the adapter card and the read-out software on the data acquisition PC. It also supplies a $3.3 \mathrm{~V}$ LVDS signal to the FE-I3 chips.

\subsection{The USBPix test system}

The USB ${ }^{1}$-based read-out system used for the characterization and calibration of ATLAS pixel devices is the USBPix setup [34] (Figure 4.1), developed by SiLab at Universitat Bonn in Bonn, Germany. The hardware is made of two custom built interlocking boards called the Multi-IO board and the module adapter card. The system will be adapted for use with the new ATLAS pixel readout system FE-I $4^{2}$ which is currently under development. The system will eventually replace the existing calibration and read-out system, TurboDAQ.

The Multi-IO board contains a USB micro-controller chip which takes commands

\footnotetext{
${ }^{1}$ Universal Serial Bus

${ }^{2}$ Front-End Iteration 4
} 
passed from a computer to the board and converts it into commands to be processed by the FPGA ${ }^{3}$ in which some of the ATLAS pixel device control and data processing are implemented using hardware. Full ATLAS pixel modules and single-chip assemblies are connected to the Multi-IO board via the module adapter board. The module adapter card adapts signal levels, and adjusts for signal propagation delays. Two types of module adapter cards exist: The single-chip adapter board, and the quad-chip adapter board. All USBPix boards are shipped with a single-chip adapter.

The entire setup is connected to a measurement PC via USB and is controlled by a customized version of the STcontrol application, which is a modified version of the pixel read-out software used by the ATLAS collaboration. The software is still in development, but contains the functionality necessary to operate ATLAS pixel devices and write data read from the devices to file. In addition the software allows direct access to all chip registers and contains automated procedures for the calibration and characterization of the devices and adjust various chip parameters.

The device is capable of generating triggers using hit information, and accepting external triggers for reading out hit information, but a loopback circuit does not exist to make the device self-triggering. The software is currently not able to interface with external software for use in a testbeam environment, but that functionality is under development.

\subsection{USBPix test-stand hardware}

The USBPix system is made up of several devices which work in tandem to provide a complete read-out system. The USBPix board and a pixel device are typically sold bundled together, but several power supplies and a computer are also necessary for the system to function. A digital and analog power supply provide the power necessary

\footnotetext{
${ }^{3}$ Field Programmable Gate Array
} 


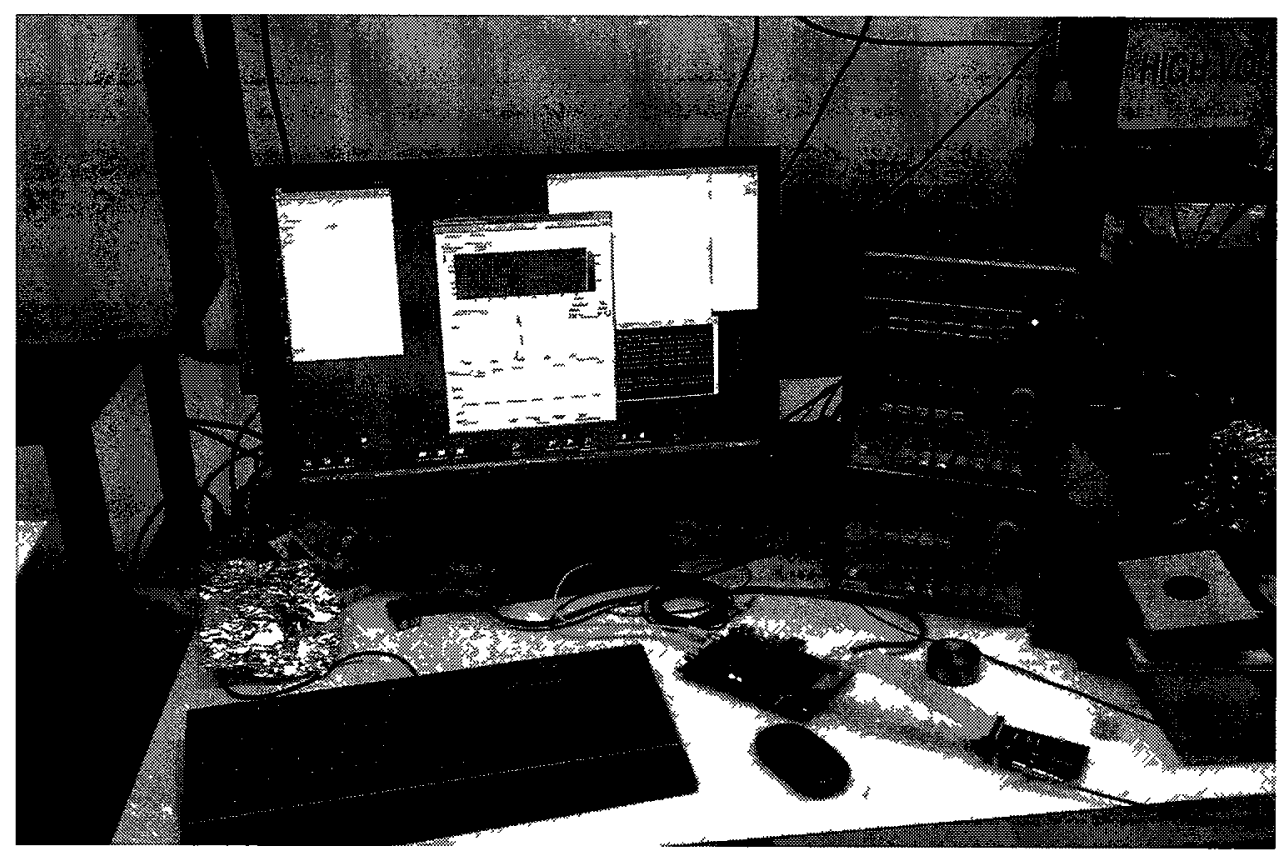

Figure 4.2: Carleton University USBPix test-stand.

for the front-end of the pixel read-out electronics to function, and a high-voitage power supply provides the sensor bias to the pixel sensor material.

The Carleton USBPix test-stand (shown in Figure 4.2) consists of a Keithley $2410 \mathrm{HV}$ power supply which provides the bias voltage across the sensing material. 2 Agilent E3631A LV power supplies provide the analog (VDD-A) and digital (VDD-D) power necessary for the respective front-end circuits outlined in Section 3.2 to work. The USBPix board is used for interfacing the read-out PC and compatible FE-I3 devices, as well as provide the $3.3 \mathrm{~V}$ required for the $\mathrm{LVDS}^{4}$ signal to the pixel device via the USB cable.

Provided with each USBPix board is a single-chip FE-I3 pixel device with planar silicon as a sensing material. The Carleton setup received pixel device 10-7A. The charge injection circuit was measured using the on-board test-paddles to have $C_{\text {low }}$, $C_{h \imath}$, and $V_{c a l}$ values of $7.909 \mathrm{fF}, 43.380 \mathrm{fF}$ and $-0.888 \mathrm{mV}$ respectively. The two

\footnotetext{
${ }^{4}$ Low-voltage differential signaling
} 
capacitor values and calibration voltage are necessary for the calibration of the chip using STcontrol.

The Carleton test-stand has been interfaced with an external trigger system consisting of a scintillator pad which outputs to a $\mathrm{TLU}^{5}$ made up of a discriminator, and a $\mathrm{NIM}^{6}$ to $\mathrm{TTL}^{7}$-compatible logic level signal conversion box (for use with the USBPix system).

\subsection{Calibration of the front-end chip}

The charge collection threshold and ToT charge readout must be tuned to known operational parameters before an ATLAS pixel device may be used as a hit reconstruction device. This is done using the calibrated charge injection circuit of the FE-I3 device. STcontrol uses a software library called PixLib which contains algorithms designed to optimize various register values on the chip. Those register values parametrize the charge threshold and ToT values that the chip produces on a pixel by pixel basis.

\subsubsection{Sensor Bias}

Scanning the bias voltage applied to a sensor and measuring the resulting leakage current will show the behaviour of the chip. This scan was performed on the Carleton University chip 10-7A starting at $0 \mathrm{~V}$ and increasing in $5 \mathrm{~V}$ steps until $200 \mathrm{~V}$, averaging 10 current measurements at that voltage. We can see in Figure 4.3 that at low bias voltages the sensor has a high resistance; however as the bias voltage increases to nearly $100 \mathrm{~V}$, the sensor stabilizes at a lower resistance.

This lower resistance is the optimal place to operate a sensor, ensuring maximum

\footnotetext{
${ }^{5}$ Trigger Logic Unit

${ }^{6}$ Nuclear Instrumentation Module

${ }^{7}$ Transistor-transistor logic
} 


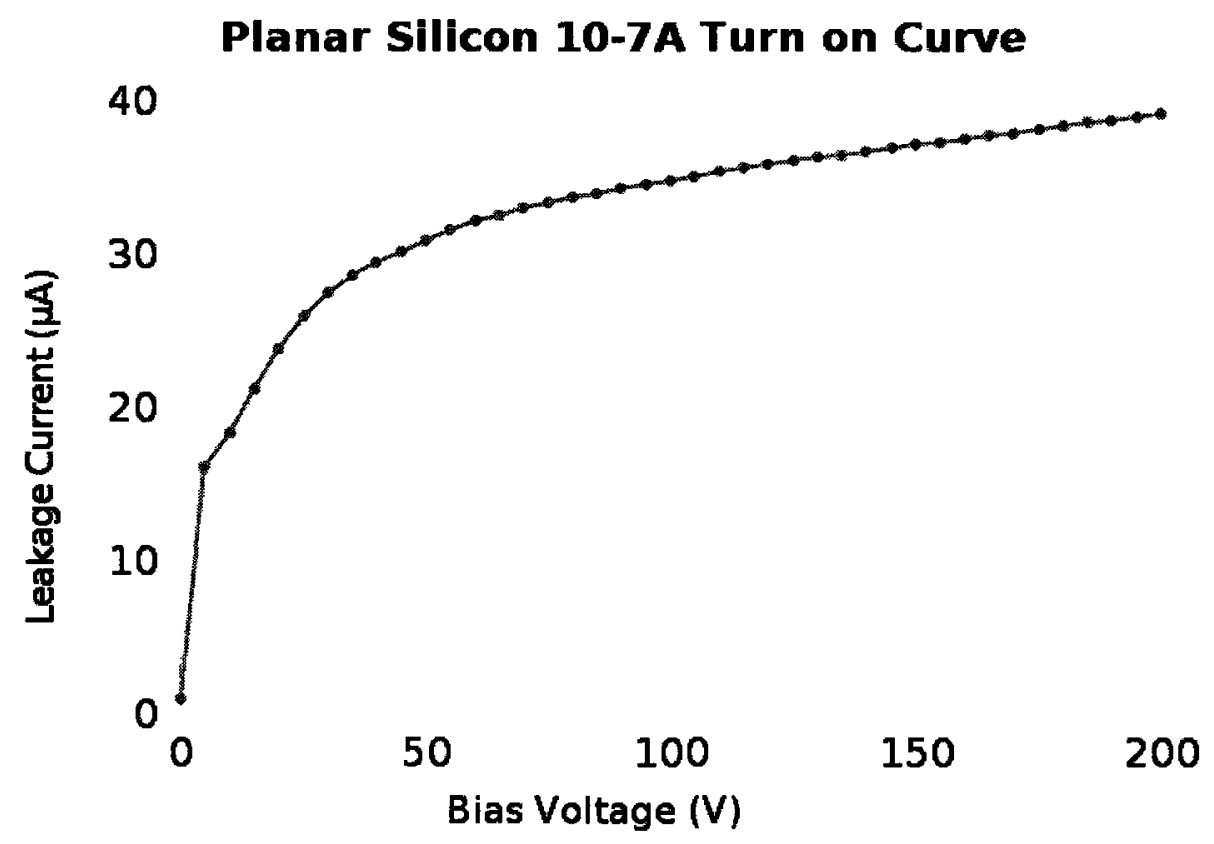

Figure 4.3: I-V turn on curve for the planar silicon chip 10-7A. The applied bias voltage on the sensor was incremented from 0 to $200 \mathrm{~V}$ in $5 \mathrm{~V}$ steps, 10 current measurements were taken and averaged at each step, and were plotted against the respective voltage. At low bias voltages $(<50 \mathrm{~V})$ sensor resistance is high, but stabilizes at a lower value when a semiconducting state is reached shortly thereafter. A stable operating bias voltage of $150 \mathrm{~V}$ was chosen. At higher bias voltages a breakdown voltage can be reached which causes damage to the sensor; thankfully no evidence of sensor breakdown can be seen.

charge mobility without reaching the breakdown voltage of the sensor. Fortunately, no evidence of sensor breakdown can be seen. The bias voltage of $150 \mathrm{~V}$ was selected to improve the stability of the sensor at high fluences.

\subsubsection{Threshold Adjustment}

Only pixels with a charge deposit registered as over a designated threshold are considered for readout by the electronics of the pixel chip. The threshold for each pixel are controlled by two types of $\mathrm{DAC}^{8}$ registers on the chip called the $\mathrm{GDAC}^{9}$, and the

\footnotetext{
${ }^{8}$ Digital to Analog Converter

${ }^{9}$ Global threshold DAC
} 
individual pixel TDACs ${ }^{10}$. The former allows for a coarse control of the chip's charge threshold, and the latter allows for a fine pixel by pixel correction.

A DAC tune is an automated process which repeatedly modifies a specific onboard register over a given interval with a given step size. At each iteration the tune performs a specific test which optimizes sensor performance and calibrates the sensor to meet a targeted parameter. Tunes exist to automate the process of sensor optimization and calibration.

The threshold of a pixel is obtained by injecting several calibration charges and measuring the fraction of detected hits. This process is repeated over a charge interval, and the fraction of hits which fire the discriminator are fitted against injected charge. In the case of an ideal discriminator we expect the response to be a step function; due to random noise effects however, the real response is typically skewed and is given by a standard error function defined as

$$
P_{h \imath t}(Q) \equiv \frac{1}{\sqrt{\pi}} \int_{0}^{\infty} \exp \left(\frac{Q-Q_{\text {thresh }}}{\sqrt{2} \sigma_{\text {nosse }}}\right) d Q
$$

where $Q_{\text {thresh }}$ is the discriminator threshold, and $\sigma_{\text {thresh }}$ is the noise of the system. The point in the fitted distribution which corresponds to a $50 \%$ hit fraction is returned as the charge threshold for the given pixel, and the fitted value of $\sigma_{\text {nosse }}$ is returned as the noise of the pixel.

Tuning the GDAC and TDAC values modify the charge value of the discriminators on each pixel to a targeted value, inputted by the user at the time of calibration. Each pixel's mean charge threshold and noise is then stored and presented in a way to assist the user in identifying problematic regions, and dealing with them accordingly.

A GDAC tune, shown in Figure 4.4, with a targeted charge threshold of $4000 \mathrm{e}$ managed to obtain a mean value of $4130 \mathrm{e}$, and has a spread of $570 \mathrm{e}$. This means

\footnotetext{
${ }^{10}$ Pixel threshold DAC
} 
that only $1 \sigma$ of the 2880 pixel channels had a threshold that fell within 570 e of the targeted value. This large spread of charge thresholds was then reduced to $22 \mathrm{e}$ using several TDAC tunes, shown in Figure 4.5, which also moved the mean threshold closer to the target value at 3999 e and results in a mean charge threshold variation of $0.6 \%$ across the entire detector; furthermore, Figures 4.5 (a) and (c) show no localized regions in the sensor where the pixels are dead (infinite thresholds) or hot (thresholds overly low or near $0 \mathrm{e}$ ).

The electronic noise found in the calibration of chip 10-7A was found to be approximately 170 e varying by 7 e across the detector before the TDAC tune as seen in Figure 4.6, and 160 e varying by 7 e across the detector after a TDAC tune as seen in Figure 4.7.

The tuned noise hitmap and channel distribution show that most pixels operate with a fairly uniform noise centred around $162 \mathrm{e}$, and pixels around the edges of the chip operate with a noise of approximately 190 e or higher. To maximize the signal to noise ratio in the sensor, pixels whose noise exceeds 180 e were masked. This can be done in STcontrol using various built in functions, specifically by turning off the pixel hit-bus and read-out mask bits. These pixels will still be calibrated fully during the ToT calibration process.

\subsubsection{ToT Adjustment}

Due to the digital nature of the ATLAS pixel read-out system it is necessary to emulate the analog measurement of charge deposited in a sensor by measuring the amount of time a signal spends over threshold (ToT), as explained in Section 3.2.

As with the charge threshold, two types of chip registers exist to give a chipaverage $\left(\mathrm{IF}^{11}\right)$, or a pixel-by-pixel $\left(\mathrm{FDAC}^{12}\right)$ adjustment of the feedback current on

\footnotetext{
${ }^{11} I_{F}$ or Feedback Current

${ }^{12}$ Feedback Current DAC
} 

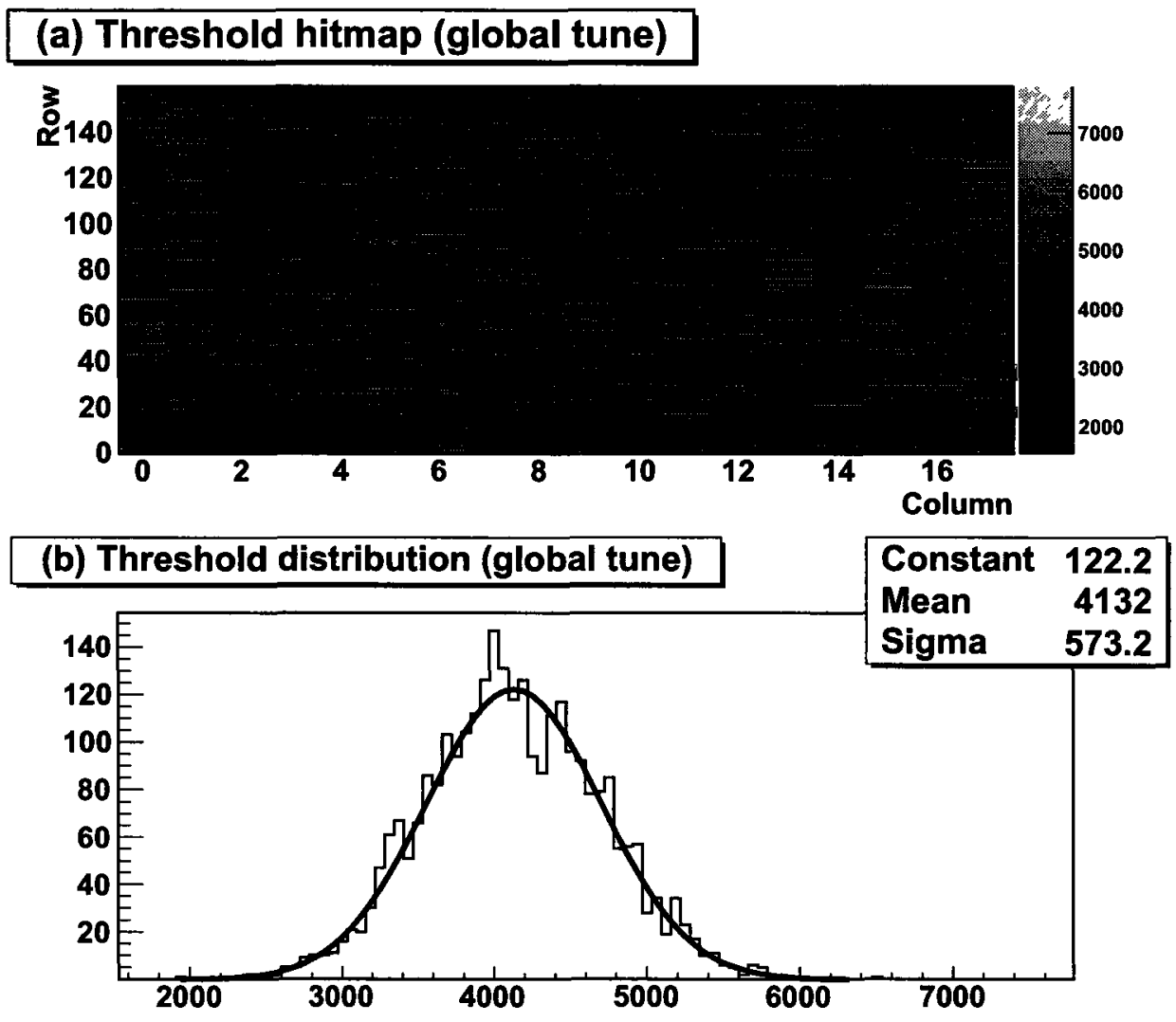

(c) Threshold vs Channel (global tune)

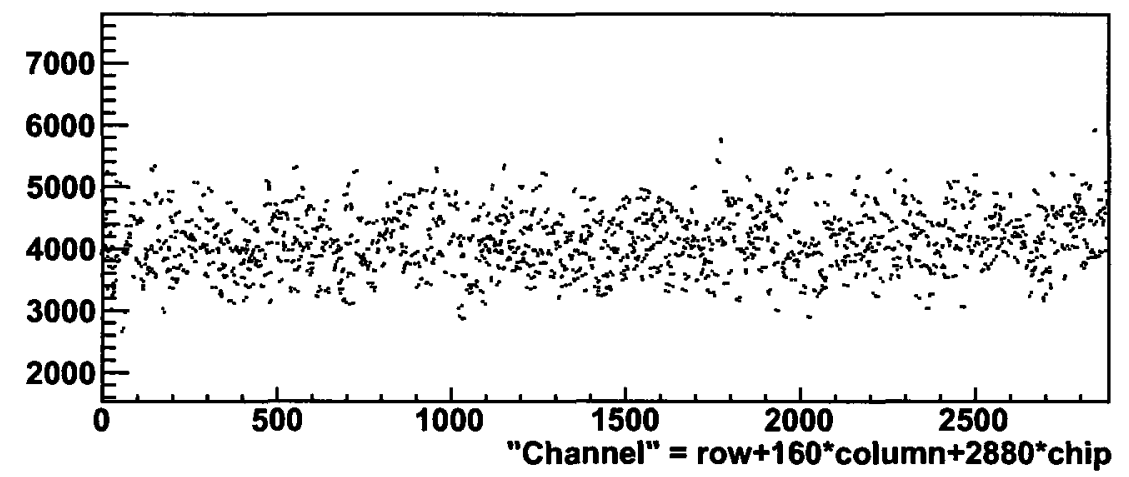

Figure 4.4: Pixel charge threshold mean for the globally tuned planar silicon FE-I3 chip 10-7A. The top plot is a hitmap of measured threshold values for each pixel. The middle plot shows the distribution of measured thresholds for all pixels and is clearly Gaussian distributed with $\mu=4132 \mathrm{e}$ and spread $\sigma=573 \mathrm{e}$; this is consistent with the desired value of $4000 \mathrm{e}$, but the distribution needs to be narrowed for a proper ToT calibration. The bottom plot is a scatter plot of the measured pixel thresholds vs channel ID (where channel $=$ row + col $\cdot 160$ ). 


\section{(a) Threshold hitmap (pixel tune)}
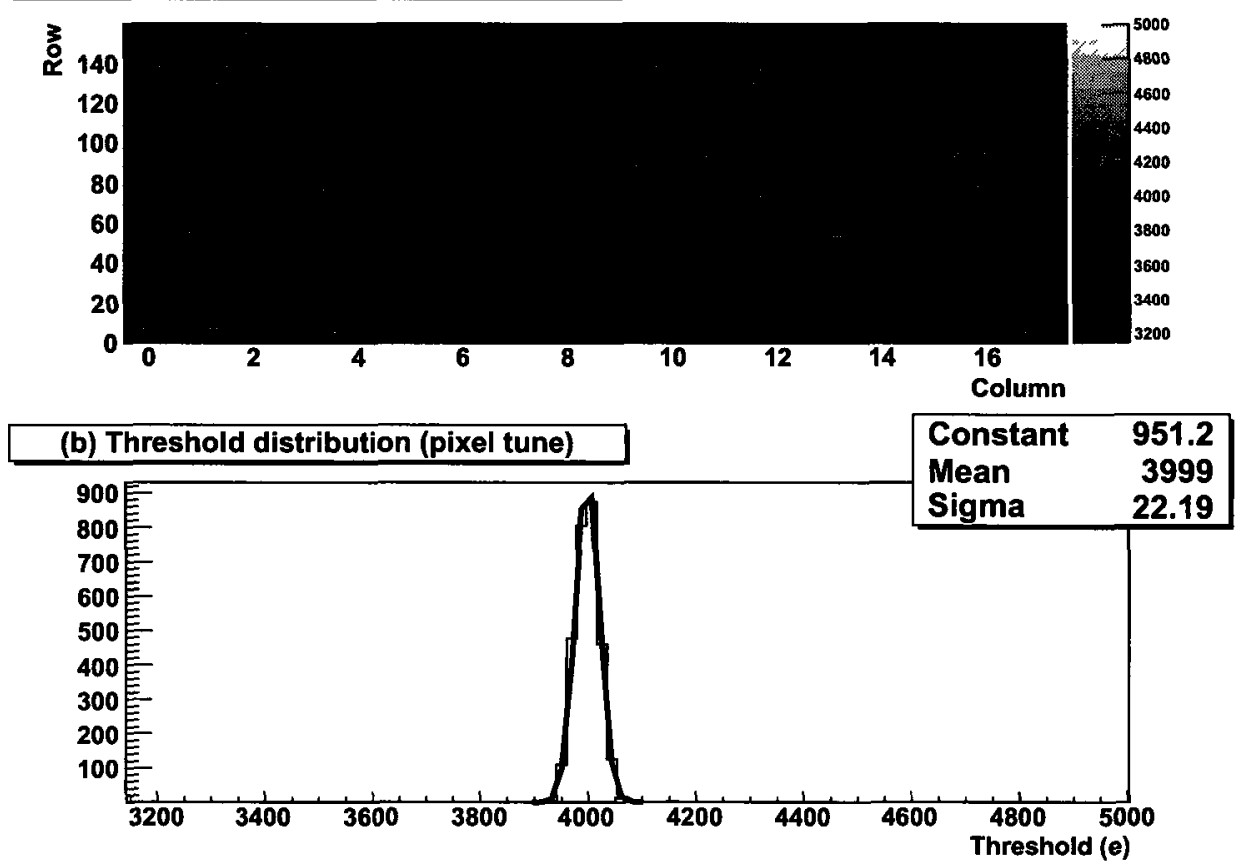

(c) Threshold vs Channel (pixel tune)

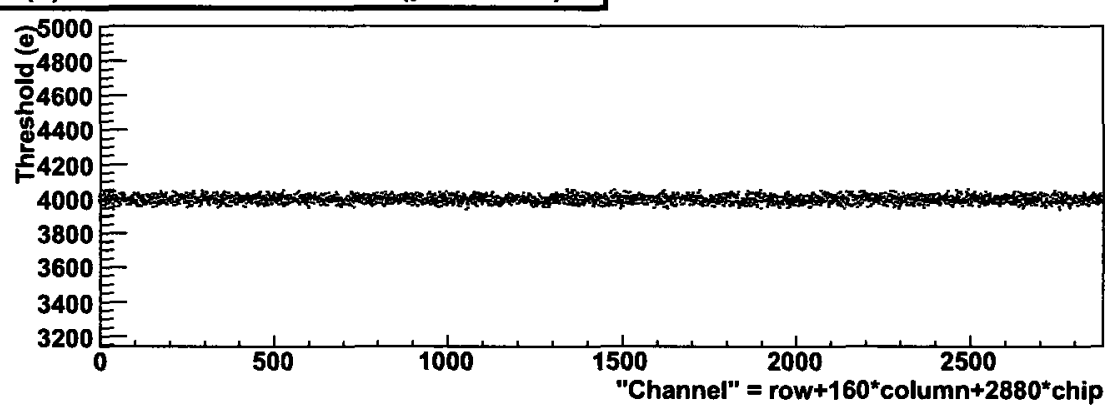

Figure 4.5: Pixel charge threshold mean for the completely tuned planar silicon FE-I3 chip 10-7A. The top plot is a hitmap of measured threshold values for each pixel. The middle plot shows the distribution of measured thresholds for all pixels and is clearly Gaussian distributed with $\mu=3999$ e and spread $\sigma=22 \mathrm{e}$, consistent with the desired value of 4000 e. The bottom plot is a scatter plot of the measured pixel thresholds vs channel ID (where channel $=$ row + col $\cdot 160$ ) shows a relative increase in threshold consistency across all channels when compared to Figure 4.4. 


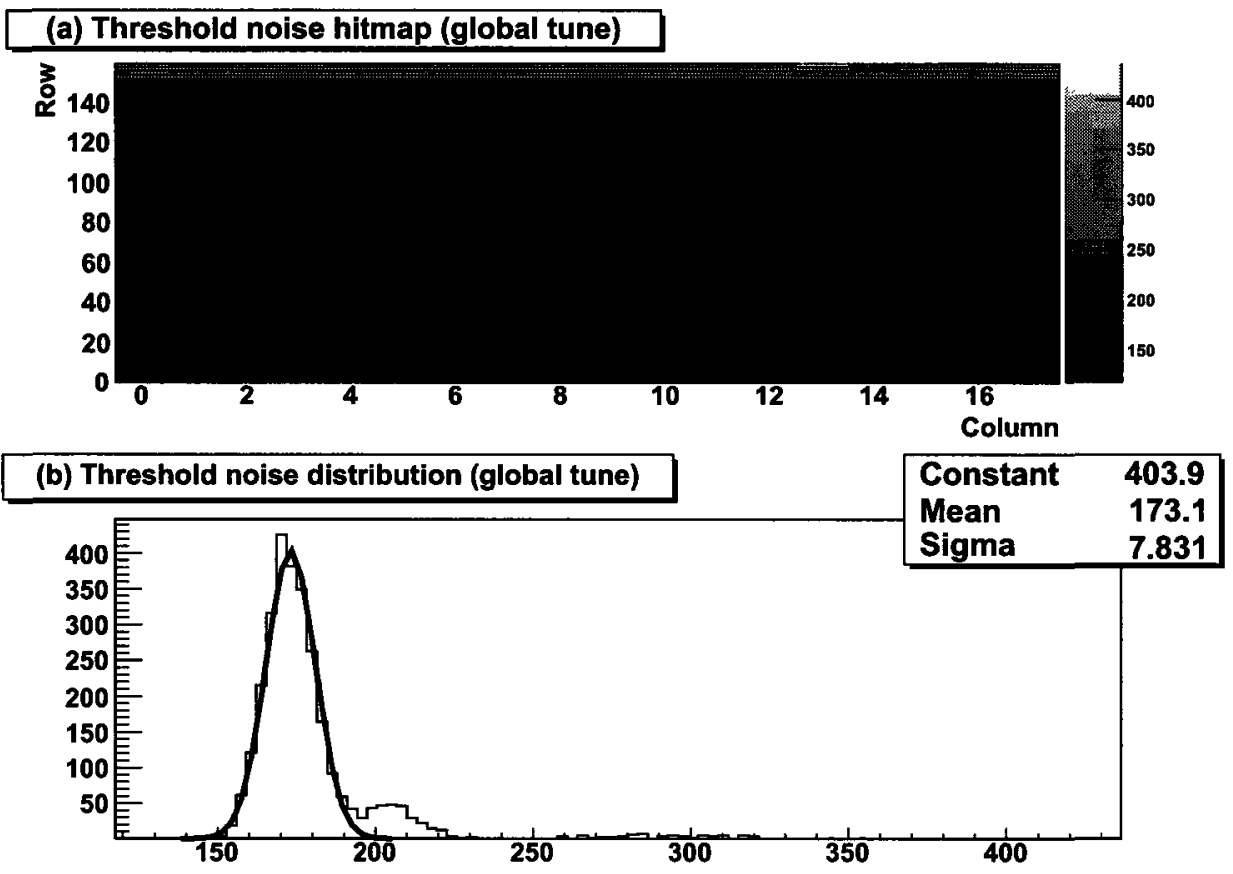

(c) Threshold noise vs Channel (global tune)

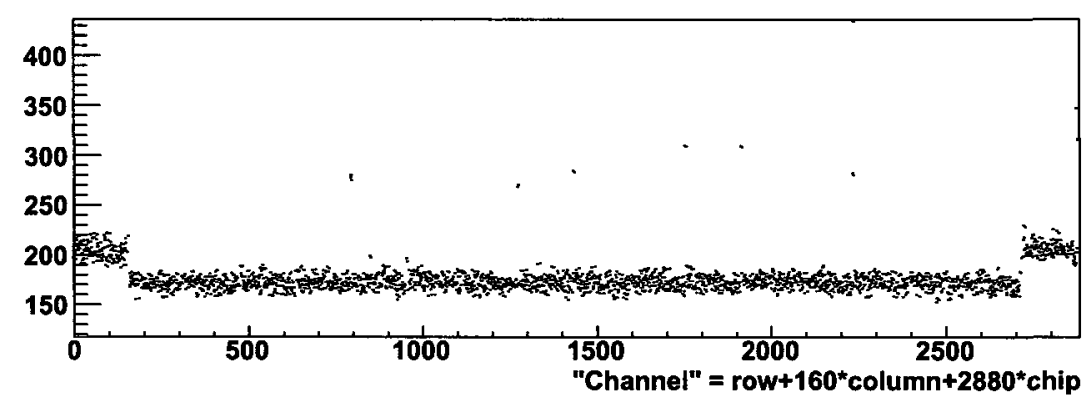

Figure 4.6: Pixel charge threshold noise for the globally tuned planar silicon FE-I3 chip 10-7A. The top plot displays the measured charge thresholds in a pixel hitmap, and increased noise on the perimeter of the chip can clearly be seen. The middle plot shows the charge threshold noise distribution. The mean pixel noise was determined to be $\mu=173 \mathrm{e}$, and varies only by $\sigma=7 \mathrm{e}$ across the layout of the chip. A double peak structure shows the higher noise pixels on the edge of the chip, and provides a numerical value to remove noisy pixels via a mask. The bottom plot shows the channel noise distribution, where a clear periodic structure allows for easy identification of noisy channels. 


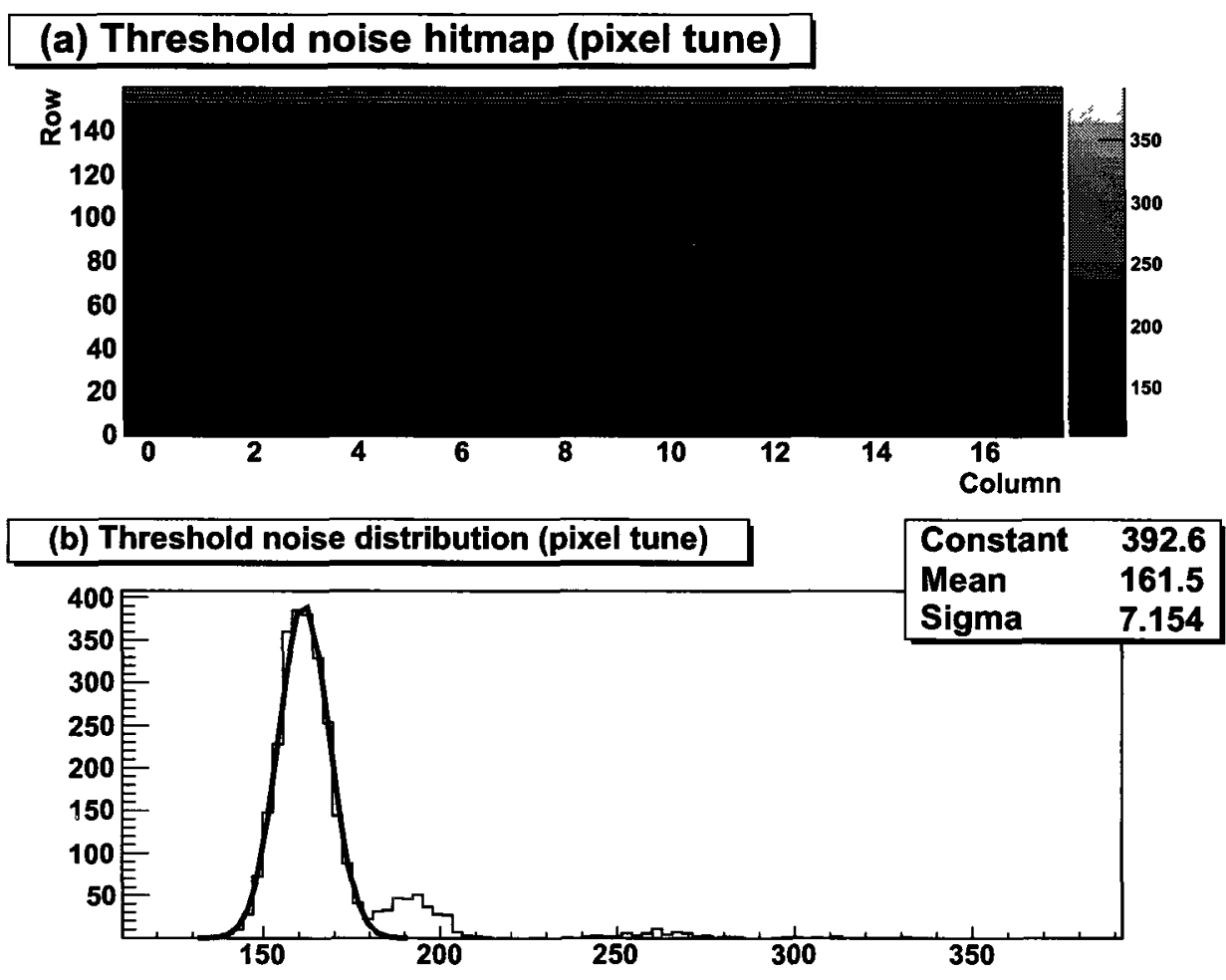

\section{(c) Threshold noise vs Channel (pixel tune)}

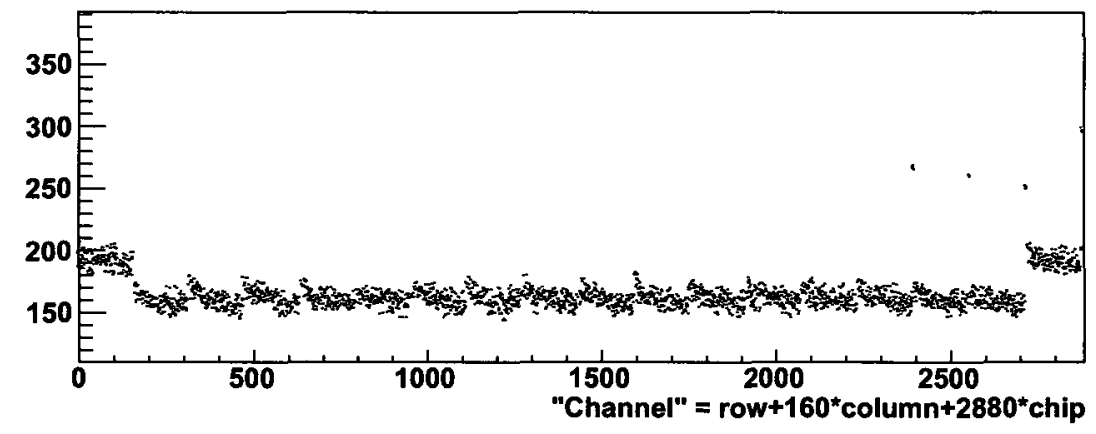

Figure 4.7: Pixel charge threshold noise for the completely tuned planar silicon FE-I3 chip 10-7A. The top plot displays the measured charge thresholds in a pixel hitmap, and increased noise on the perimeter of the chip can clearly be seen. The middle plot shows the charge threshold noise distribution. The mean pixel noise was determined to be $\mu=162 \mathrm{e}$, and varies only by $\sigma=7 \mathrm{e}$ across the layout of the chip. A double peak structure shows the higher noise pixels on the edge of the chip, and provides a numerical value to remove noisy pixels via a mask. The bottom plot shows the channel noise distribution, where a clear periodic structure allows for easy identification of noisy channels. 
each pixel's charge sensitive amplifier. An IF or FDAC tune will inject a calibration charge and measure the ToT response for each pixel, the IF or FDAC values will then be modified within a given range to determine the register value which optimizes the ToT response to a given bunch crossing count for that injected charge.

Both the injected calibration charge and the ToT response are specified prior to the tune. The uncertainty on the injected charge depends on the components of the charge injection circuit shown in Figure 3.4. The injection circuit capacitors $C_{l o}, C_{h}$ and the injection voltage gradient $V_{c a l}$ must all therefore be measured and provided to STcontrol prior to an IF or FDAC tune.

The ToT response for a silicon chip of $250 \mu \mathrm{m}$ thickness is commonly set to 30 $\mathrm{BC}$ counts over threshold for an injected charge of $20 \mathrm{ke}$. For diamond the response is typically targeted to 30 counts over threshold for $10 \mathrm{ke}$. These targets were chosen because they are the expected charge deposition for minimum ionizing particles passing through their respective materials, and they provide sufficient resolution to reconstruct varying amounts of charge deposited in the detector.

An IF tune was performed on planar silicon chip 10-7A to a target ToT of $30 \mathrm{BC}$ for an injected charge of $20 \mathrm{ke}$, and the results of that tune can be seen in Figure 4.8. The ToT response for each chip is again shown to be centred near the target with a mean of $29.6 \mathrm{BC}$, and is widely distributed with a spread of $1.6 \mathrm{BC}$. Non-linearities are also visible in Figure 4.8 (c), where the mean ToT seems to decay linearly with channel ID. The response was then improved via an FDAC tune shown in Figure 4.9, which shifted the mean ToT to 29.9 BC with a spread of 0.5 BC. Fortunately the hitmaps show no localized ToT response deviations, and the sensor can be considered tuned.

After a chip has been tuned to produce a specific threshold and ToT response it is necessary to perform a ToT calibration. A ToT calibration is a scan which parametrizes a chip's ToT response as a function of injected charge for each pixel 


\section{(a) ToT Mean hitmap (global tune)}
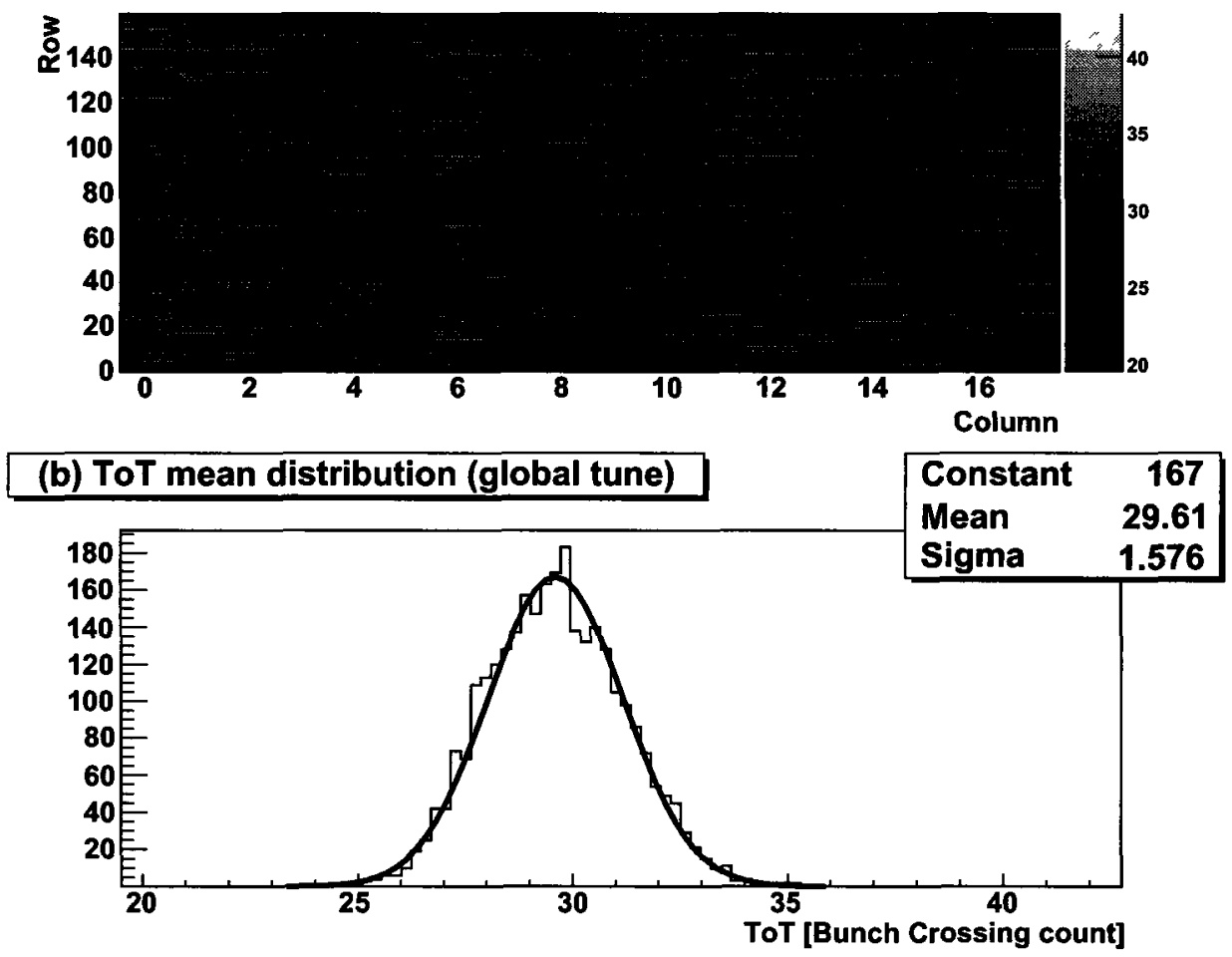

\section{(c) ToT mean vs Channel (global tune)}

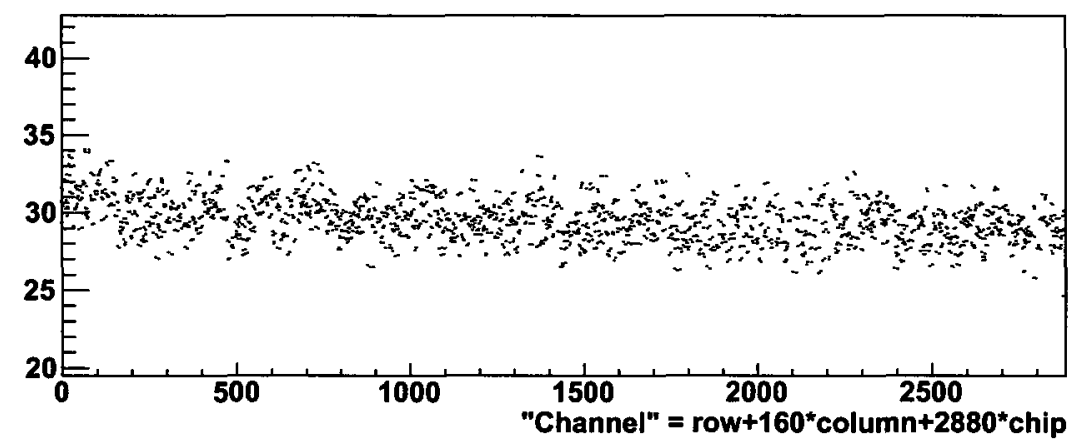

Figure 4.8: ToT response for planar silicon FE-I3 chip 10-7A. The top plot shows a hitmap of the measured ToT response across all pixels. To T was tuned to an average charge count of $\mu=29.9 \mathrm{BC}$ counts over threshold at $20 \mathrm{k}$ electron charges, and varies by $\sigma=1.6 \mathrm{BC}$ counts across the entire chip. 
(a) ToT mean hitmap (pixel tune)
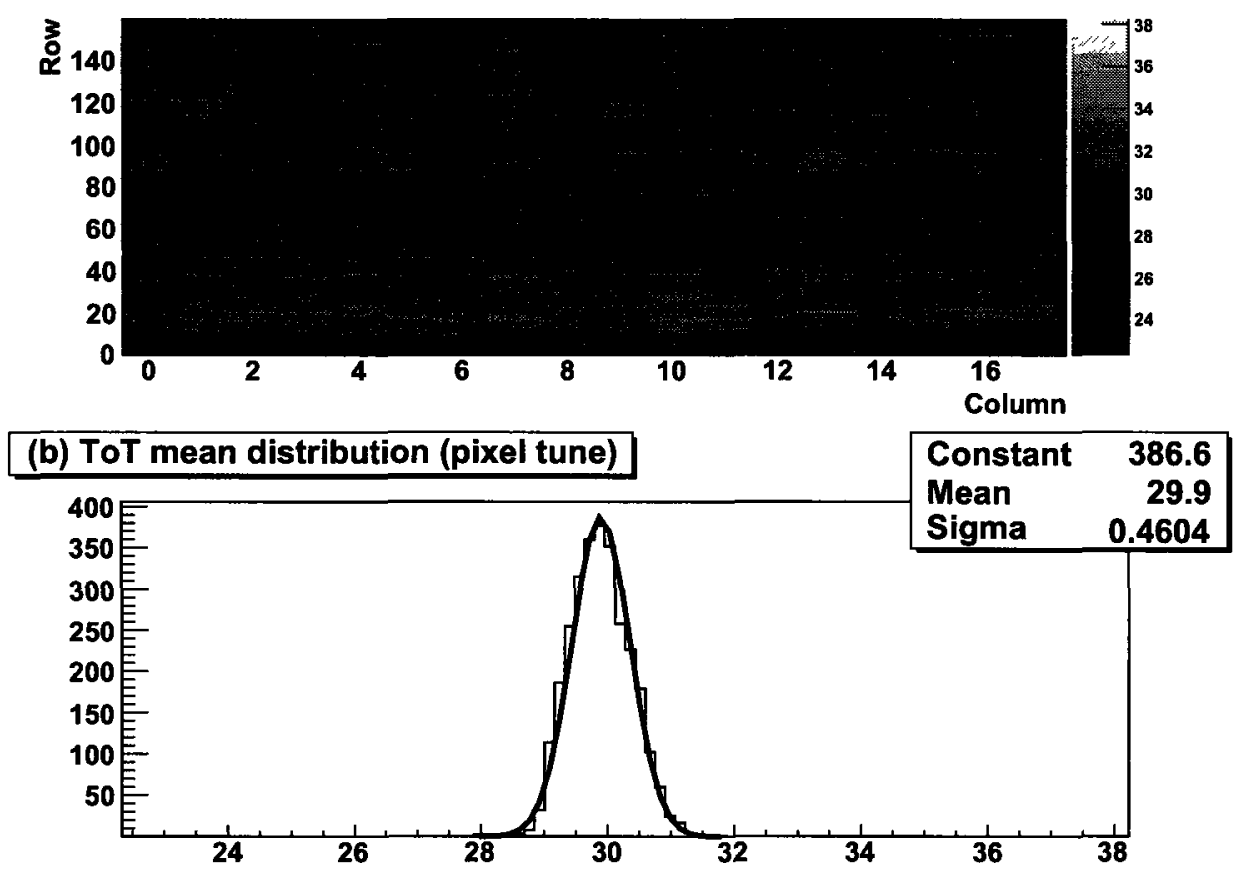

(c) ToT mean vs channel (pixel tune)

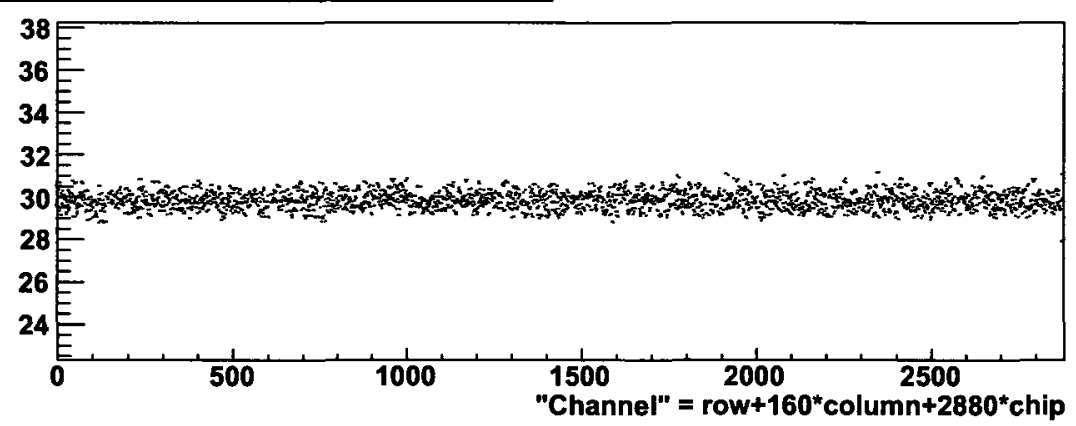

Figure 4.9: ToT response for planar silicon FE-I3 chip 10-7A. ToT was tuned to an average charge count of $\mu=29.9 \mathrm{BC}$ counts over threshold at $20 \mathrm{k}$ electron charges, and only varies by $\sigma=0.4 \mathrm{BC}$ counts across the entire chip. 
individually. This information gives a more precise ToT to charge conversion for every single pixel, and is necessary to perform analyses which depend on charge collection. One would expect the relation between injected charge and ToT response to be linear, but non-linearities are introduced by the analog part of the front-end electronics. This is particularly true for charge near the threshold. The relation between injected charge and ToT is best represented as a three-parameter function given by

$$
Q_{\mathrm{nj}}=\frac{P_{3} \cdot\left(\mathrm{ToT} / P_{1}\right)-P_{2}}{1-\mathrm{ToT} / P_{1}}
$$

where $Q_{\mathrm{n} \mathrm{J}}$ is the charge injected into the pixel, and $P_{\imath}$ are shape defining parameters which are left floating in the fit. This calibration accounts for the non-linearities in the ToT response of the chip at ToT counts near the threshold $(<10$, and has an uncertainty of approximately $10 \%$.

\subsubsection{Chip calibration algorithm}

Software algorithms called tunes in the PixLib package exist to automatically obtain desired device performance. Several register tunes are required in order to properly calibrate a pixel chip. Tunes automatically scan ranges of register values and record device performance to find the values which correspond to a pre-defined target. The order of the tunes performed are chosen to maximize their efficiency and efficacy.

An IF tune is performed to globally set the feedback current of all pixels to roughly the target ToT at a given injected charge. The ToT measured for a calibration charge for all pixels are typically gaussian distributed, and random properties of each pixel will result in a widely spread distribution.

A GDAC tune is performed to set the charge threshold of all chips to roughly the target threshold. This is performed after the IF tune because changing the feedback 
current will affect the charge threshold due to the setup of the read-out electronics. The GDAC is a global register and the resulting threshold distribution for the chip will be on the order of 700 electron charges. The charge threshold for a diamond sensor is typically targeted to be 1700 electron charges.

A TDAC tune is performed to bring individual pixels closer to the target threshold. This scan can reduce the spread of the charge threshold to between 40 - 60 electron charges. An FDAC tune is then performed to reduce the mean ToT distribution similarly. The FDAC tune is performed after the TDAC tune to ensure a proper threshold is established. Without a well defined threshold, the target ToT will not be determined efficiently. A TDAC tune is then repeated to negate the changes to the threshold induced by changing the feedback current in each pixel by the FDAC tune.

\subsection{Generating a pixel mask}

Due to the difficult nature of producing pixel detectors it is possible that upon production, or with normal wear and tear, certain pixels will become damaged and either cease functioning (dead pixels), or become overly sensitive (hot pixels). It is necessary before use to determine which pixels are no longer functioning and mask them. STcontrol gives the user many options when it comes to masking pixels, but the most effective is to remove the pixel from use entirely by turning off the pixel's pre-amp, and not reading the pixel out.

As was shown in Figure 4.7 (a), the pixel device provided with the USBPix had several noisy pixels which needed to be masked near the edge of the active area. A mask was created which turned off all pixels with a noise greater than $180 \mathrm{e}$. 


\subsection{Source scans}

When the USBPix is used in conjunction with an external trigger such as our scintillator and a radioactive source, STcontrol is capable of reading out events from the pixel read-out electronics and storing vital information such as ToT counts, hit locations, and trigger timing in a human readable text file using a source scan. Source scans can be used to test the read-out electronics and ensure that the ToT spectrum (and thus the charge deposited) in the presence of known ionizing radiation matches up with the expected charge deposition of the source. Source scans run continuously until a limit specified prior to beginning the scan is reached.

\subsubsection{Trigger Delay}

The amount of time it takes an electrical signal to propagate through a series of devices is in practice non-zero, and processes such as amplification and discrimination typically introduce delays which must be accounted for in a real-time application such as external triggering.

STcontrol reads out all hits found in a pixel device in a 16 bunch crossing window after a trigger is received. Hits received before and after the allotted window are not considered for read-out purposes. Hits are flagged with a 4-bit counter value called a $\mathrm{BCID}^{13}$ which denotes how many bunch crossings after the trigger received that hit was first registered as over threshold. A trigger which is generated simultaneously in a scintillator with hits in a pixel device may reach the pixel device much later than the $16 \mathrm{BC}$ window if sufficient delay is introduced, and so it is necessary to compensate for that by properly setting the L1 delay register.

The L1 delay is an 8-bit register which tells the front-end to hold hit information for the stored value $n$ bunch crossings to compensate for trigger delay. If this value

\footnotetext{
${ }^{13}$ Bunch Crossing IDentifier
} 
is not properly set, then the hits recorded from the triggering particle(s) will be lost when the 16 bunch crossing window is met. It is advantageous to set the L1 delay such that the triggers received are in the middle of the read-out window - doing so ensures that as few hits are lost as possible at the edges of the read-out window.

\subsubsection{Results}

Source scans at Carleton have been performed using Sr-90. A source scan was performed on the planar silicon chip provided with the USBPix board (serial number 10-7A). The scan took approximately 16 hours to collect $30 \mathrm{k}$ hits due to the activity of the source. The source was uncollimated to allow for a shorter run time, and increase charge sharing between pixels.

Charge deposition by beta particles from Sr-90 decays via $\beta$ decay with an energy of $0.55 \mathrm{MeV}$ into $\mathrm{Y}-90$ which decays via $\beta$ decay with an energy of $2.28 \mathrm{MeV}$, resulting in a total decay energy of $2.83 \mathrm{MeV}$. A decay energy of $2.83 \mathrm{MeV}$ for an electron corresponds to $\beta \gamma \approx 5.4$, which places it squarely in the MIP region of the $d E / d x$ curve (see Figure 2.1). We therefore expect the total charge deposition from Sr$90 \beta$ particles in a $250 \mu \mathrm{m}$ silicon sensor to be on the order of $27 \mathrm{ke}$. The $\beta \gamma$ of the $\beta$ particles is too low to effectively estimate the MPV of charge deposited using Equation 2.4; but a general rule of thumb is that the MPV to mean ratio for silicon is approximately 1.4, which would place the estimated most probable charge deposition in a $250 \mu \mathrm{m}$ silicon sensor at $19 \mathrm{ke}$.

In order to consider events which contain more than one pixel which collected charge, an analysis package is provided with the USBpix software. The code groups hits by event, and requires all active pixels be within 2 pixels of all other hits, and occur within $4 \mathrm{BCs}$ of all other hits in the cluster. Charge sharing in pixels allow for more precise position reconstruction and a higher charge collection efficiency, so chips are calibrated and oriented in such a way that charge sharing is maximized during 


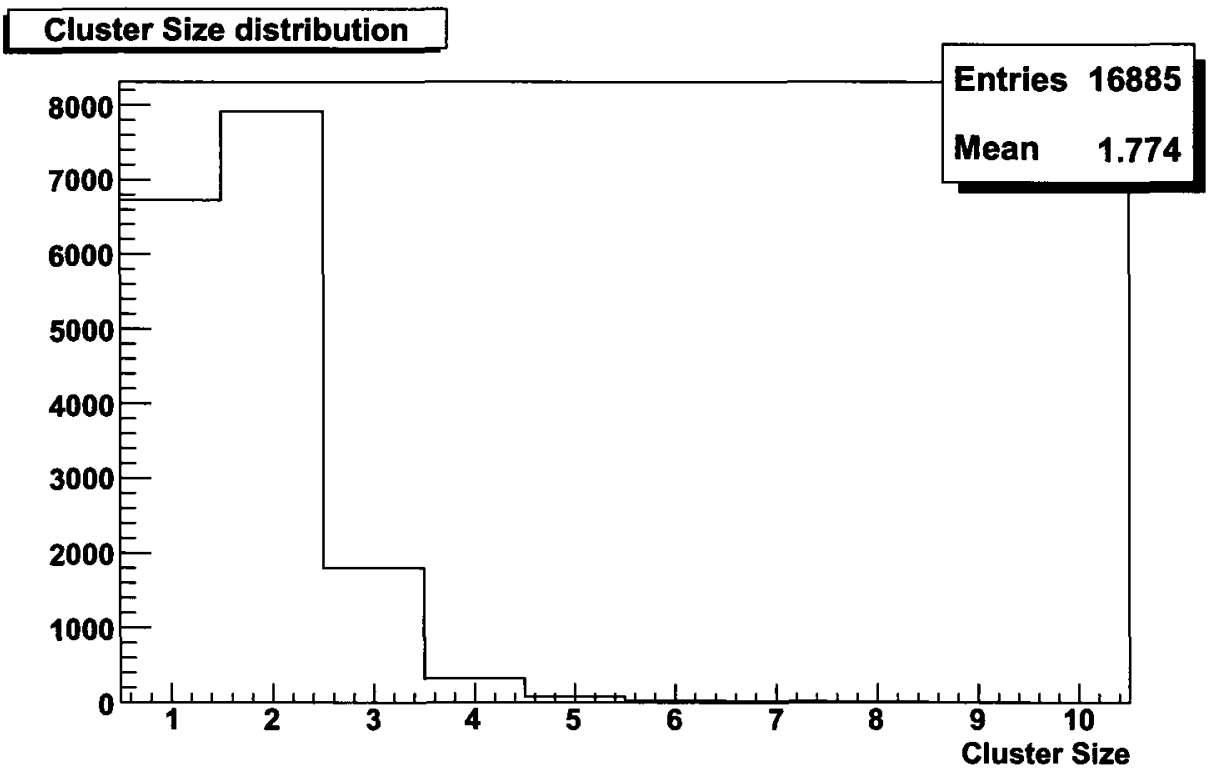

Figure 4.10: Cluster size distribution for Sr-90 source scan using planar silicon FEI3 chip 10-7A. Cluster size corresponds to the number of hits which are adjacent to each other in a common event. Because the source was uncollimated, charge sharing between pixels is common. Clusters with more than one pixel over threshold are more likely to collect the entire charge deposited in the sensor than a cluster with only one registered hit.

runs. We can see from Figure 4.10 that charge sharing occurs in more than $50 \%$ of events, and since most clusters have no more than 3 hits, we do not gain significant statistics by considering cluster sizes larger than 3 .

The map of all reconstructed cluster locations can be seen in Figure 4.11, and were received on average $9 \mathrm{BC}$ after a trigger according to Figure 4.12. The sharp LVL1 distribution confirms that events received are not due to random noise in the sensor. The source spot was adjusted to the middle of the sensor, away from any noisy pixels and the edge of the sensor to ensure no signal was lost.

Finally we consider the collected charge in the sensor to ensure that we are indeed triggering on incident beta particles rather than noise. The ToT distribution for all cluster sizes as well as clusters of size 1,2 and 3 can be seen in Figure 4.13 as the ToT distributions for CS1, CS2 and CS3 respectively. The conversion from ToT counts to 


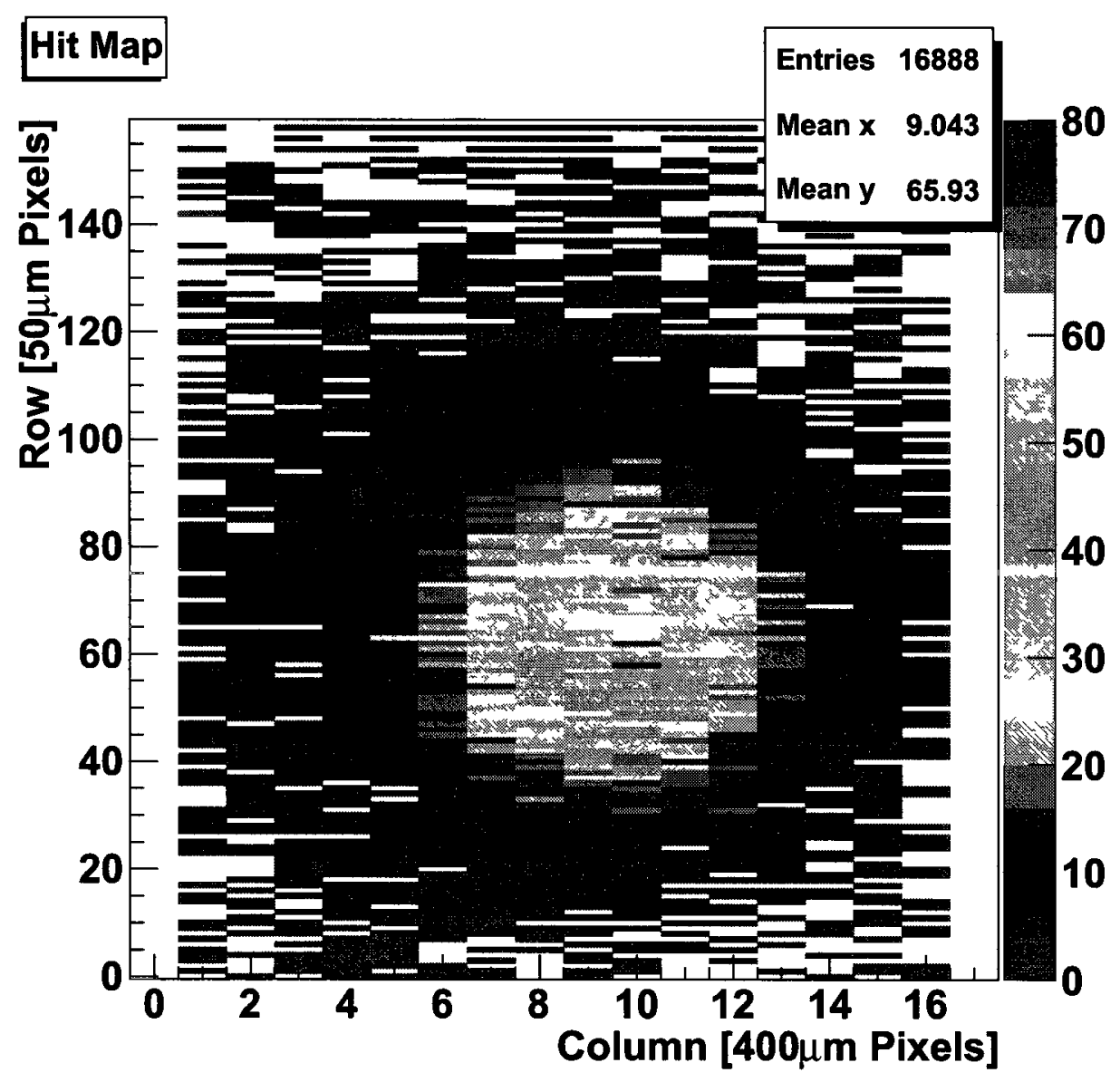

Figure 4.11: Hit-map for a 30k hit Sr90 source scan with planar silicon chip 10-7A. Notice the hot spot formed under the source in the middle of the sensor. Pixels in columns 0 and 17, as well as several rows near the top edge of the chip were masked in the calibration process due to excessive noise. Events with multiple localized hits were reconstructed into hit clusters and are shown as a single entry for the purpose of considering total charge deposited in the sensor. 


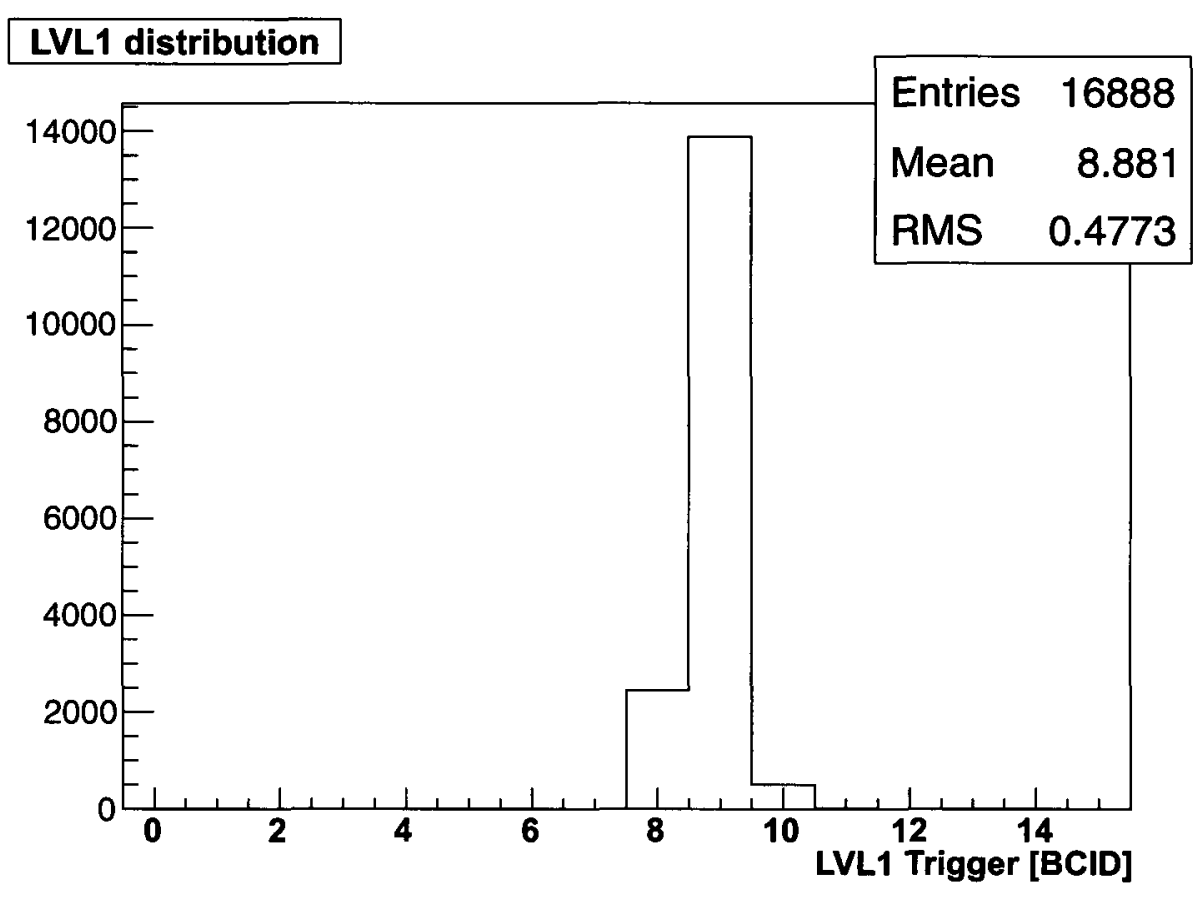

Figure 4.12: Level 1 trigger distribution for Sr-90 source scan using planar silicon FE-I3 chip 10-7A. The Level 1 trigger corresponds to which bunch crossing window after the trigger was received a given hit falls in. Only hits which are observed within $16 \mathrm{BC}$ of the trigger are kept to minimize noise in the sensor. The majority of hits were observed approximately 7 bunch crossings after a trigger was received. Hit BCIDs are calculated accounting for signal propagation delays using the L1 delay described in Section 4.5.1 
charge can be seen in Figure 4.14 using equation 4.2.

The ToT response from the source scan is shown in Figure 4.13, and shows clearly formed Landau distributions for various cluster sizes. The ToT distribution is indicative of charge deposition in the sensor as expected from MIPs. Clusters with a single hit (CS1) have structure forming around $5 \mathrm{BC}$ which is absent from larger cluster sizes (CS2 and CS3). The origin of low charge events are believed to correspond to noise in the sensor.

The charge distribution from Sr-90 is expected to follow a Landau-Gaussian convolution [13]. If the sensor is functioning as expected, then we would expect the data to fit to that distribution with the parameters of the distribution matching those discussed at the beginning of this section. Fitting to the data we obtain a most probable charge deposition of $\Delta_{p}=14 \pm 1$ ke across all cluster sizes, which corresponds to a $28.6 \%$ percent difference between the expected $\Delta_{p}=19 \mathrm{ke}$ and the observed value. This can be attributed in part to the underestimation of the collected charge from the ToT calibration uncertainty, and also to charge loss in the sensor.

It is important to note that the most probable charge collected increases with cluster size. One would expect that single hit events would correspond to the lowest charge collection efficiency of all cluster sizes as a maximum of 4 ke of charge may be lost per adjacent pixel which does not register as over threshold. This is the most common source of charge loss in a sensor, and plays a large roll when considering charge collection efficiency. Higher charge collection efficiency corresponds to less noise in the sensor, provides a stronger basis for position reconstruction.

It is conceivable that larger cluster sizes may consist of a hit which registered as over threshold due to random noise in the sensor, coupled with one or more signal events; if this were true, one would expect the ToT distributions for larger cluster sizes to peak at $\Delta_{p, n-1}+Q_{\text {thresh }}$, where $\Delta_{p, n-1}$ is the MPV for the lower cluster size and $Q_{\text {thresh }}$ is the value of the charge threshold for the pixel fired. This would correspond 

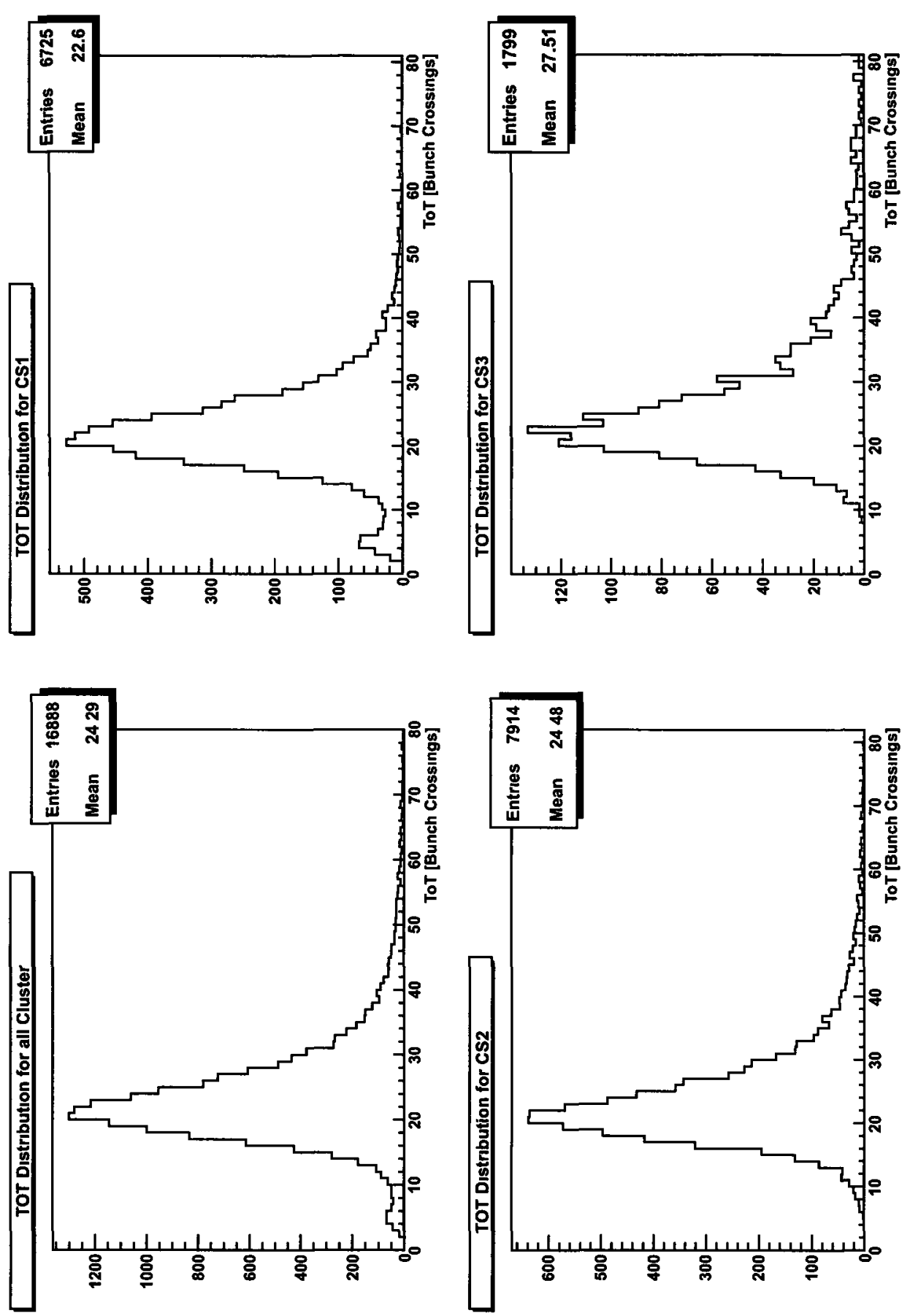

Figure 4.13: ToT response for Sr-90 source scan using planar silicon FE-I3 chip 10-7A. Clear Landau distributions are visible, corresponding to the expected ToT distribution for MIPs depositing charge in a semiconducting sensor. The properties of the Landau distribution should be proportional to the properties of the particles which injected charge, as well as how much charge was injected in the sensor. Plots have been rotated to increase size. 

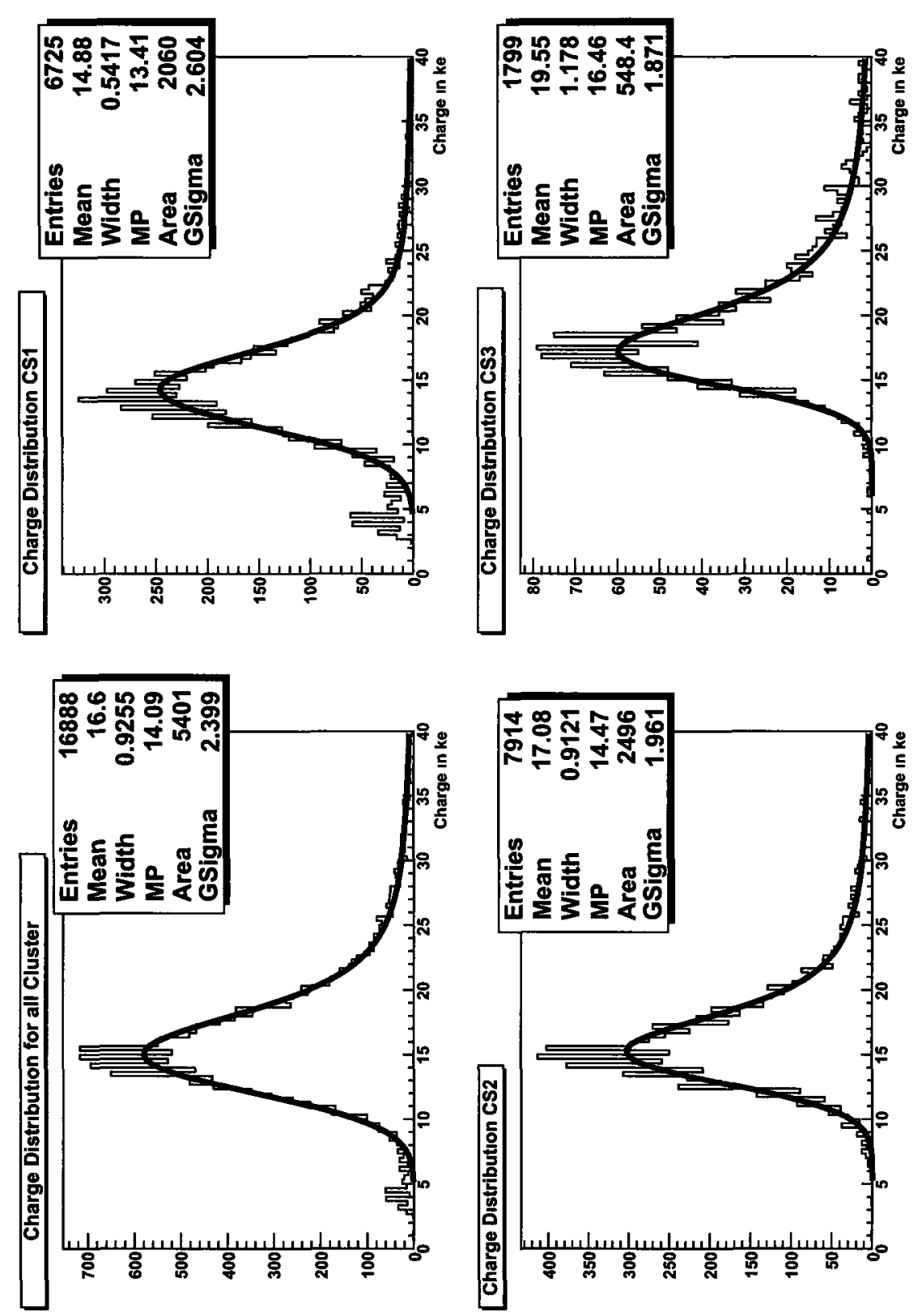

Figure 4.14: Charge deposition for planar silicon FE-I3 chip 10-7A. Charge deposited in each pixel was calculated using the ToT calibration obtained prior to the scan. Fitted functions are convolutions of Landau and Gaussian distributions. Charge reconstruction efficiency increases as the number of pixels registered over threshold increases, which is shown in the increase of the measured mean charge deposited for increased cluster sizes (CS). Plots have been rotated to increase size. 
to an MPV shift of approximately $6 \mathrm{BC}$, or $4000 \mathrm{e}$, and thankfully this does not appear to be the case.

Upon the consideration of the above data, it has been shown that the test-stand setup at Carleton University is capable of performing the calibration and characterization of FE-I3 sensor materials for use with the USBPix read-out system. With further upgrades to the read-out software STControl and testing, the setup may be appropriate for device read-out in a test-beam scenario. 


\section{Chapter 5}

\section{Test-beam at CERN}

In order to fully characterize sensor performance it is necessary to test devices at the operation energies of our detectors. These tests use a reference beam telescope capable of measuring particles from a mono-energetic beam. The beam telescope should be able to reconstruct the position and arrival time of incoming charged particles, and this information can be compared to the position and arrival times measured in the DUT $^{1}$. These tests allow us to directly study the effects of charge sharing, position resolution, and the efficiency of the detector under various operational parameters such as bias voltage, angle of incidence, and irradiation. Beam tests at CERN were conducted at the SPS ${ }^{2}$ in Prévessin, France. A $120 \mathrm{GeV} / \mathrm{c}$ pion beam was used to emulate the environment predicted to occur in the inner ATLAS detector.

I played a role in the execution of these tests and the analysis of the resulting data. I was present at CERN during the summer of 2009 and I took on several duties with two test beams: I produced several $\mathrm{DQM}^{3}$ tools which I then used to perform a diagnostic analysis of the collected data and determining their feasibility for analysis (discussed below). I was also responsible for the calibration and testing of beam tests for two full pCVD diamond modules, one irradiated to $10^{16}$ protons $/ \mathrm{cm}^{2}$ and one not

\footnotetext{
${ }^{1}$ Device Under Test

${ }^{2}$ Super Proton Synchrotron

${ }^{3}$ Data Quality Management
} 
using the BAT.

During these tests I accumulated a working knowledge of how to debug the telescope used (and FE-I3 devices), and was one of the most involved shifters of the 4-man team tasked with the test. I was responsible for all DQM, converting collected data into a usable format, data storage, and analysis of the data collected using my own tools. The telescope was also an unsupported device, which is nearing the end of its usage in beam tests. I was responsible for learning how to debug the system, and ensure that all data collected was not lost to one of the many possible malfunctions at a reasonably high frequency.

\subsection{Telescope}

\section{Bonn-ATLAS Telescope}

The beam telescope used in past trials is called the BAT ${ }^{4}$ shown in Figure 5.1, and consists of two scintillators $(5 \times 20 \mathrm{~mm}$ and $30 \times 10 \mathrm{~mm})$ which enclose up to a maximum of 6 sillicon strip detectors called BAT modules. A full description of the BAT and its capabilities are available in [35]. Currently only BatMods 1-3 and 6 are positioned in the telescope, and BatMod 2 is non-functioning due to a power supply issue.

The telescope data-rate is limited in part by the dead-time of the system, a minimum of $132 \mu \mathrm{s}$. The data rate also depends on the trigger rate of the system, but for a setup using 4 BAT modules and a single DUT the typical data rate which can be achieved is on the order of $4.5 \mathrm{kHz}$ - well above the data-taking rate of conventional VME-based read-out systems.

The passage of particles through the telescope is detected by the coincidence of the two scintillators, and triggers the recording of detected hits in the BatMod planes

\footnotetext{
${ }^{4}$ Bonn-ATLAS Telescope
} 


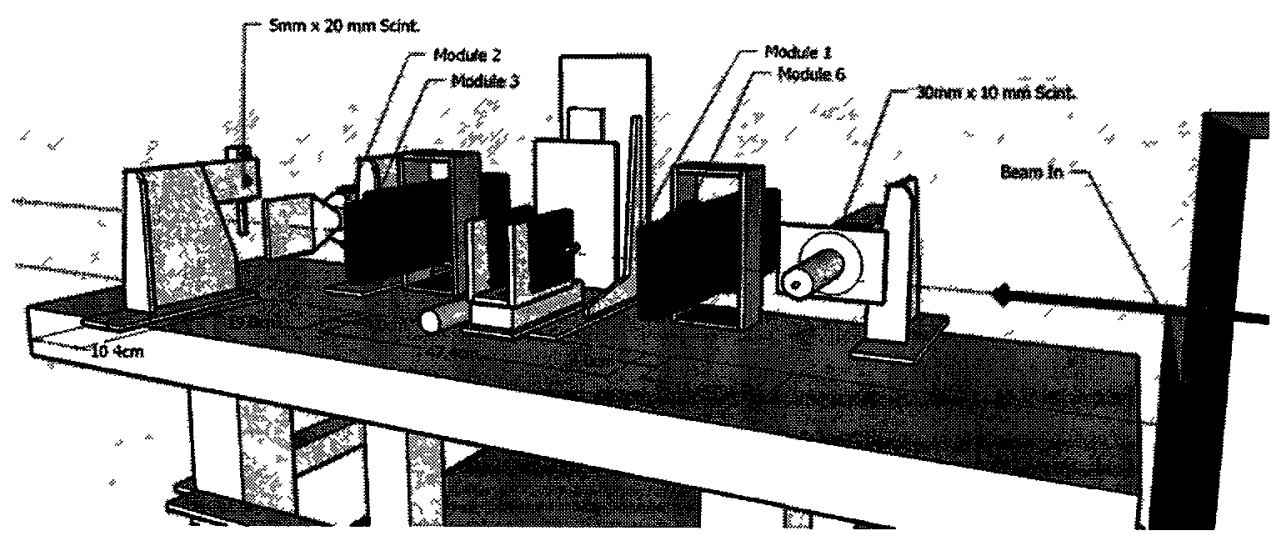

Figure 5.1: Bonn-ATLAS Telescope (BAT) scale model. 4 silicon strip BAT modules are used with up to 2 devices under test (or DUTs). 2 scintillators on either side of the telescope are used to trigger on events passing through the active areas of the DUTs. Image courtesy of Joshua Moss. Used with permission.

and the DUTs. The data collected from a trigger is called an event, and may contain several hits in each plane. Sets of events which occur without changes to the $\mathrm{DAQ}^{5}$ or setup of the telescope are referred to as a run, and runs are typically limited to some finite number of events to make the experiment more robust against the corruption of data.

The arrival time of particles in the DUT is given by the low response time of the scintillation detectors, and the position of the charged particle in the DUT can be inferred from the position of registered hits in several telescope planes by reconstructing the particle's trajectory (track). Factors which affect track resolution in the telescope are the physical limitations of hit reconstructions in the telescope planes, and multiple scattering in the air or detector plains. As the effect of multiple scattering is inversely proportional to particle energy, the effect can be minimized by using high-energy beams in the test.

A major issue with the BAT formed some time between August 2007 and July

\footnotetext{
${ }^{5}$ Data Acquisition software
} 
2008. Extended runs result in event/hit ID miscounts, causing de-synchronizations between active planes in the telescope mid-run without warning. This desynchronization causes hits to be associated with the incorrect event IDs, and can link noise hits to events selected as signal. The cause of this de-synchronization has yet to be identified; though they can be identified through analysis, a work around has yet to be successfully implemented.

The Bonn-ATLAS telescope was used in all RD42 pixel test-beams from 2006 to 2009. The above device failures, combined with a loss of support from the creating institution, have resulted in limitations causing the BAT to fall out of favour with the pixel R\&D groups. The current preferred beam telescope for beam tests at CERN is the EUDET telescope. I was tasked with analyzing the old data, and developing replacement analysis tools for data taken using the BAT. I have been present for two beam tests using the EUDET telescope, and have played a large role in providing shifter expertise to the group. I have not taken part in any data analysis from those tests.

\section{BAT modules}

BAT modules consist of double sided, AC-coupled silicon strip sensor manufactured by Hamamatsu photonics. Each side contains $64050 \mu \mathrm{m}$ strips, and the sides are at a stereo angle of $90^{\circ}$. This corresponds to a sensitive area of $3.2 \times 3.2 \mathrm{~cm}^{2}$, and a spatial sensitivity of $14.4 \mu \mathrm{m}$ with binary hit information, and $2.9 \mu \mathrm{m}$ [35] using corrections based on charge shared between pixels. Time limitations prevented the implementation of a charge sharing algorithm, limiting the sensor's resolution to that of a binary sensor. 


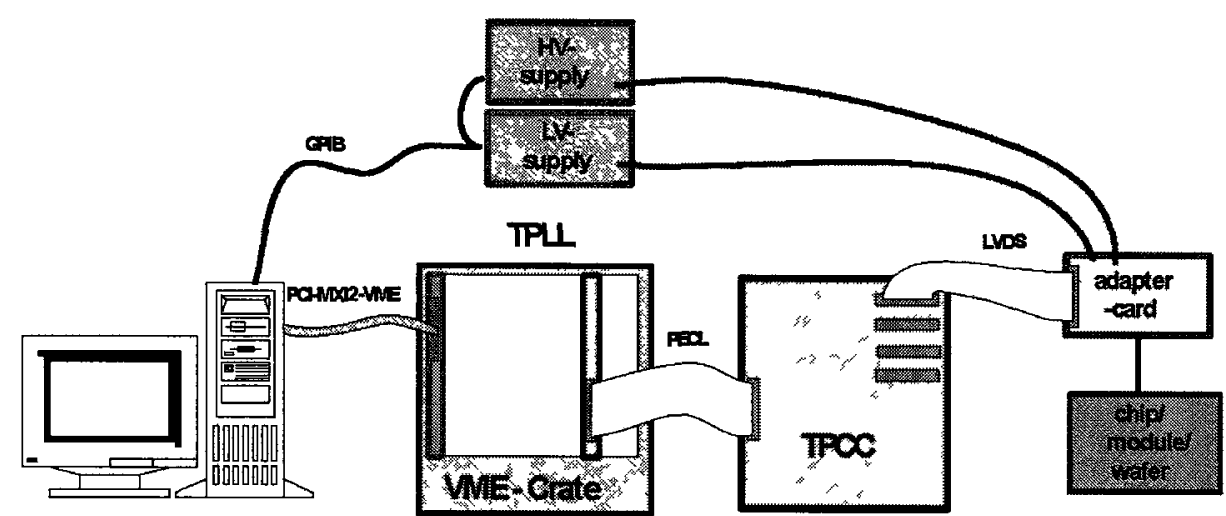

Figure 5.2: Overview of the TurboDAQ setup.

\subsubsection{Data acquisition}

Data acquisition for the BAT telescope is done using a custom built VME-based hardware and software suite Read-out for the DUTs are handled using a software and VME-based hardware package called TurboDAQ. The TurboDAQ system was developed at Lawrence Berkley National Laboratory in Berkley, California, and is very similar to the USBPix system described in section 4.1. The TurboDAQ setup can be seen in Figure 5.2.

The TurboDAQ system uses two custom pieces of hardware called a TPLL ${ }^{6}$ (similar in role to the MultiIO board) and a $\operatorname{TPCC}^{7}$ (similar in role to the adapter card). The TPLL generates all control signals used in the operation of the device, provides an interface for the external trigger, and houses an FPGA and buffer for hit information.

The TPCC adjusts signal levels and accounts for propagation delays as well as multiplexing the connection for up to 4 separate devices. A limitation of the system is that 4 devices may be operated and controlled simultaneously, but the hit information from only one device at a time can be processed. The entire system is connected to the read-out PC via a VME-bus interface, and controlled using the TurboDAQ software

\footnotetext{
${ }^{6}$ ATLAS Turbo Pixel Low Level card

${ }^{7}$ ATLAS Turbo Pixel Control Card
} 
suite. The TurboDAQ software suite provides all the same functions as STcontrol for the USBPix system, but is fully developed for use in a test-beam scenario.

\subsection{Dataset summary}

I developed an analysis software suite designed specifically for use with the data collected from the BAT. The software was developed entirely by myself building upon previous analysis techniques as a basis for the programming model. The software is capable of rudimentary event selection, hit reconstruction, device alignment and track reconstruction in the BAT as well as a basic charge collection analysis in a DUT. The software has since been adopted by other members of the RD42 collaboration in an effort to extend its capabilities, and perform a more rigorous analysis of the collected data.

In order to better characterize the damage done to diamond as a function of irradiation, a single diamond sensor was considered in the analysis. The sensor chosen was a $395 \mu \mathrm{m}$ thick, single crystal CVD diamond named CD181. The sensor has undergone two $24 \mathrm{GeV} / \mathrm{c}$ proton irradiations at the CERN-PS irradiation facilities, and three beam tests have been conducted at the irradiations listed in Table 5.1. All datasets were taken with a $100 \mathrm{GeV}$ pion beam at a $0^{\circ}$ angle of incidence, and the front-end was tuned to a threshold of $1600 \mathrm{e}$ with a ToT response of $30 \mathrm{BC}$ at $10 \mathrm{ke}$.

CD181 was bump-bonded to a single chip ATLAS FE-I3 module for the duration of the beam tests listed in Table 5.1, and was bump-bonded to a new FE-I3 chip in 2009. Complications involving the sensor's metallization and To'T response have barred it from further beam tests until recently. No other CVD diamonds have been tested successfully after irradiation to date using the BAT telescope.

Unfortunately almost all calibration data from previous test-beam periods (2006 - 


\begin{tabular}{ccc}
\hline \hline Test-beam period & Bias voltage $(\mathrm{V})$ & Irradiation $\left(\right.$ protons $\left./ \mathrm{cm}^{2}\right)$ \\
\hline October 2006 & 400 & 0 \\
August 2007 & 400 & $8.5 \times 10^{13}$ \\
July 2008 & 800 & $0.7 \times 10^{15}$ \\
June 2009 & 800 & $0.7 \times 10^{15}$ \\
\hline \hline
\end{tabular}

Table 5.1: Summary of datasets taken and analyzed using scCVD diamond CD181. 2008) have been lost, and so we must rely on a more rudimentary charge reconstruction formula

$$
Q_{\mathrm{nj}}=\frac{10 k e \cdot \mathrm{ToT}}{30 \mathrm{BC}}
$$

which is highly dependent on the uncertainty of the ToT calibration at 30 bunch crossings. This introduces a higher uncertainty on a per-pixel charge measurement and does not account for non-linearities in the ToT response of the electronics; for ToT values near $30 \mathrm{BC}$ however, we can estimate the uncertainty on the injected charge as $10 \%$.

\subsection{Track reconstruction and alignment}

When reconstructing particle tracks, hit positions need to be determined with a high degree of accuracy. For detectors with a spatial resolution on the order of a few $\mu m$ it is vital to know the alignment of all detectors precisely with respect to the entire telescope, and a survey of the telescope will not provide an accurate enough depiction of that alignment. The alignment must therefore be constructed using the tracks themselves.

To accomplish the task of track reconstruction and device alignment a software 
package was developed using the ROOT framework, which is an object-oriented analysis library written in $\mathrm{C}++$ at CERN to replace its FORTRAN based predecessor PAW. Track fitting is performed using the MINUIT library, and the software's structure was modelled on a previous analysis package named $\mathrm{SiTBeAn}^{8}$, developed by M. Mathes at Bonn University.

\subsubsection{Hit clustering}

Reconstructing the location of a particle track which passed through a sensor depends on how many hits are observed during the event in question. Events with only a single hit are said to be "digital" and have a minimum spatial resolution of

$$
\sigma_{p r x e l}=\frac{d_{p t t c h}}{\sqrt{12}},
$$

which is a physical limitation of the device. Events which contain more than one pixel over threshold may be reconstructed into a cluster of hits. If two or more hits are found localized within a pixel of each other we can reconstruct the location of the track based on how much charge was deposited in each pixel. A weighted mean of $n$ hits is constructed as

$$
x_{\text {reco }}=\frac{\sum_{\imath=0}^{n} Q_{\imath} \cdot x_{\imath}}{\sum_{\imath=0}^{n} Q_{\imath}},
$$

where $x_{\text {reco }}$ is the reconstructed hit location, $q_{\imath}$ is the charge measured in the pixel and $x_{\imath}$ is the position of the hit $i$ in the event. The uncertainty on a cluster with two or more hits therefore depends on the measured charge of each hit and its uncertainty. For simplicity, it is possible to use the row and column ID of pixels as position measurements, and scale the strip/pixel sizes into physical dimensions after analysis.

The BAT telescope planes read out strips registered as over threshold (called seed

\footnotetext{
${ }^{8}$ Silab Test-beam Analysis framework
} 
strips) as well as the two adjacent strips on either side regardless of whether or not they have registered as over threshold. The signals received from those strips can be compared against each other, and any bias to the left or right of the seed can be attributed to charge sharing between strips. Charge sharing occurs when signal charge drifts between strips, or an angle of incidence exists between the sensor and the incident particle.

The resolution of the telescope planes can be optimized by taking special care during tuning. As tracks are uniformly distributed, the width $L$ of the region in which charge division can occur between two channels is estimated using the ratio between the number of single- and double-hit events. In the ideal case where the number of single- and double-hit events are equalized, this corresponds to $L \approx 10 \mu \mathrm{m}$, thus the error on hit reconstruction is halved by effectively reducing the strip pitch using the hit information.

The electronics required to enable the recording of adjacent strips is more involved than that of the FE-I3 chipset, and requires a deep understanding of the behaviour of those electronics. Measuring the amount of charge in a channel which hasn't registered as over threshold requires you to trust in the stability of the read-out electronics at low signal. If the electronics are capable of producing reliable sub-threshold measurements, then removing that functionality results in a loss of reconstructive power.

Events with hits will consist of a minimum 5 strips being read out. The seed strip, and the 4 neighbouring strips adjacent to the seed. If two strips are near each other the adjacent strips read-out may overlap, and event will consist of anywhere between 6 and 10 strips. Examples of event layouts can be seen in Figure 5.3. Only events with 5 or 6 strips read-out in all telescope planes are reconstructed, ensuring that only events with a single charged particle track are reconstructed. This selection greatly simplifies the track reconstruction process, removing the need to consider track fitting multiplicities. 

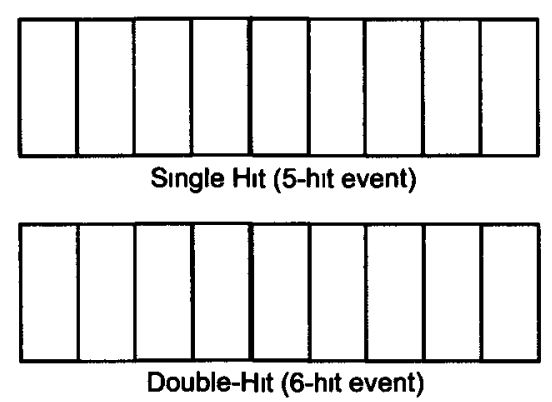

Strip not read out or over threshold

Strip read out, not over threshold

Strip over threshold, and read out

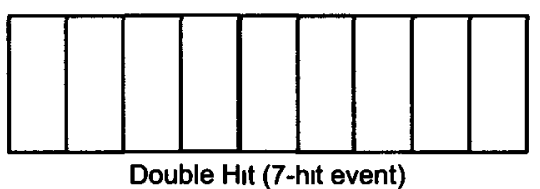

Figure 5.3: BAT hit read-out examples. Strips in BAT planes which are not registered as over threshold may be read out to provide additional hit information via charge sharing. Examples for single and double hit events where hits are registered in adjacent pixels are shown. Strips registered as over threshold are shown in red, and strips where hit information is read out are shown in red and yellow. Strips which are not read out are depicted in blue.

\subsubsection{Alignment}

Device alignment is performed by minimizing differences (residuals) between hit locations in the device, and their expected hit location from tracks reconstructed using one or more reference planes. Alignment is done in multiple stages to ensure a more accurate reconstruction of the telescope's geometry. A complete alignment would account for both device translation and rotation from the beam line; but due to time constraints, only device translations were reconstructed.

An initial guess and some knowledge of the telescope's geometry is required before alignment can be performed. Because module rotation is not considered during device alignment, special care must be taken to ensure that the bases of all devices are oriented in the same direction as a chosen reference plane. This can be done by transforming planes oriented opposite the reference basis using 


$$
x_{\imath}^{\prime}=X-x_{\imath}
$$

where $x_{\imath}^{\prime}$ is the properly oriented hit location, $X$ is the pitch of the detector's active area, and $x_{\imath}$ is the hit location as read out by the detector. Device locations along the beam axis are also needed before alignment in order to properly predict the locations of hits in devices using fitted tracks.

Alignment was performed iteratively, starting with a simple device-to-device alignment which calculates the mean offset between hits in the two planes using

$$
a_{d}=\sum_{\imath=1}^{N}\left(x_{d, \imath}-x_{r, \imath}\right),
$$

where $a_{d}$ is the alignment offset for device $d$, and $x_{d, \imath}$ and $x_{r, \imath}$ are the device and reference hit information respectively for event $\imath$ of $N$. The average alignment offset is calculated for all devices except the chosen reference plain, and is applied to the hit information as an initial adjustment. An example of the alignment offset for the X-direction of BatMod1 in the October 2006 dataset can be seen in Figure 5.4.

The next stage of device alignment fits a track to two reference planes and constructs an expected hit location in the remaining devices to compare against the measured hit location. Tracks are constructed presuming there are no deflections due to multiple scattering within the telescope using the following formula

$$
\vec{x}=\vec{a} \cdot z+\vec{b}
$$

where $\vec{x}$ is a $2 \mathrm{D}$ hit location in $x$ and $y, z$ is the position of the device along the beam axis (usually chosen such that the origin is on the DUT), and $\vec{a}$ and $\vec{b}$ are four free parameters denoting track slope and offset respectively in $x$ and $y$. Once the tracks are reconstructed, the average displacement is constructed again using 


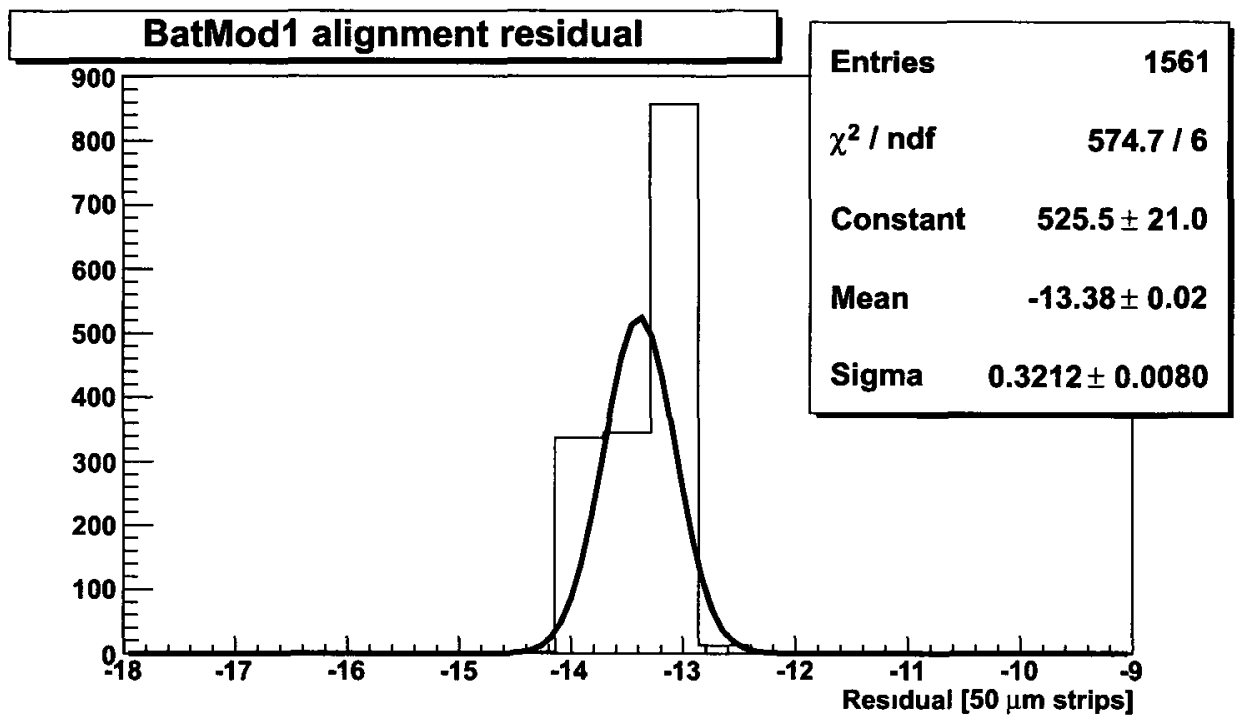

Figure 5.4: A sample residual for BatMod1 before alignment corrections have been applied shows a sharp gaussian residual centred at $\mu=-13.4$ strips with a spread of $\sigma=0.3$ strips across the considered 1500 events in the run. This residual shows that hits in BatMod1 are offset by approximately 13 strips from the reference plane BatMod6. The gaussian's mean is then used as the device's alignment offset, which is subtracted from all hits in the run on a plane by plane basis to correct for device translation with respect to the reference plane. 
equation 5.5. While the residuals for each event are being reconstructed, a running average is calculated and events which contain hits displaced by more than 5 strips from the mean are flagged, to remove them from consideration in track fitting.

This process is applied using different device permutations as the reference planes, but one plane must be kept constant to provide a frame of reference for the telescope's co-ordinate system.

The alignment of each device with respect to the $\mathrm{z}$ axis is kept constant throughout the entire process, and no offsets are determined in the code. In order to perform device alignment and track reconstruction, an initial guess of the devices' alignment along the beam-axis are required. As a test of alignment stability, the offset distribution of the October 2006 data for the X-direction of BatMod3 was plotted in Figure 5.5 , and a mean offset of $(-104.7 \pm 0.1)$ strips relative to BatMod6 was found.

When a full alignment has been reconstructed with respect to a reference plane, the device dependent alignment offsets are subtracted from individual hit measurements to produce a set of hit locations in the new telescope co-ordinate system for each individual event.

\subsubsection{Track fitting}

Track fitting in the BAT is done by performing two linear $\chi^{2}$ fits in the $x$ and $y$ directions of the telescope's co-ordinate system using equation 5.6 for every event that passes the selection criteria in the alignment phase. Tracks are reconstructed using all the devices as input, and the measured residuals for events of different cluster sizes are considered separately to simplify analysis.

The uncertainty on the reconstructed track position $\left(\sigma_{t}\right)$ is dominated by

$$
\sigma_{t}^{2}(z)=\left(\sum_{\imath} \frac{1}{\sigma_{\imath}^{2}}\right)^{2}+z\left(\frac{z_{\imath}^{2}}{\sigma_{\imath}^{2}}\right)^{2}
$$




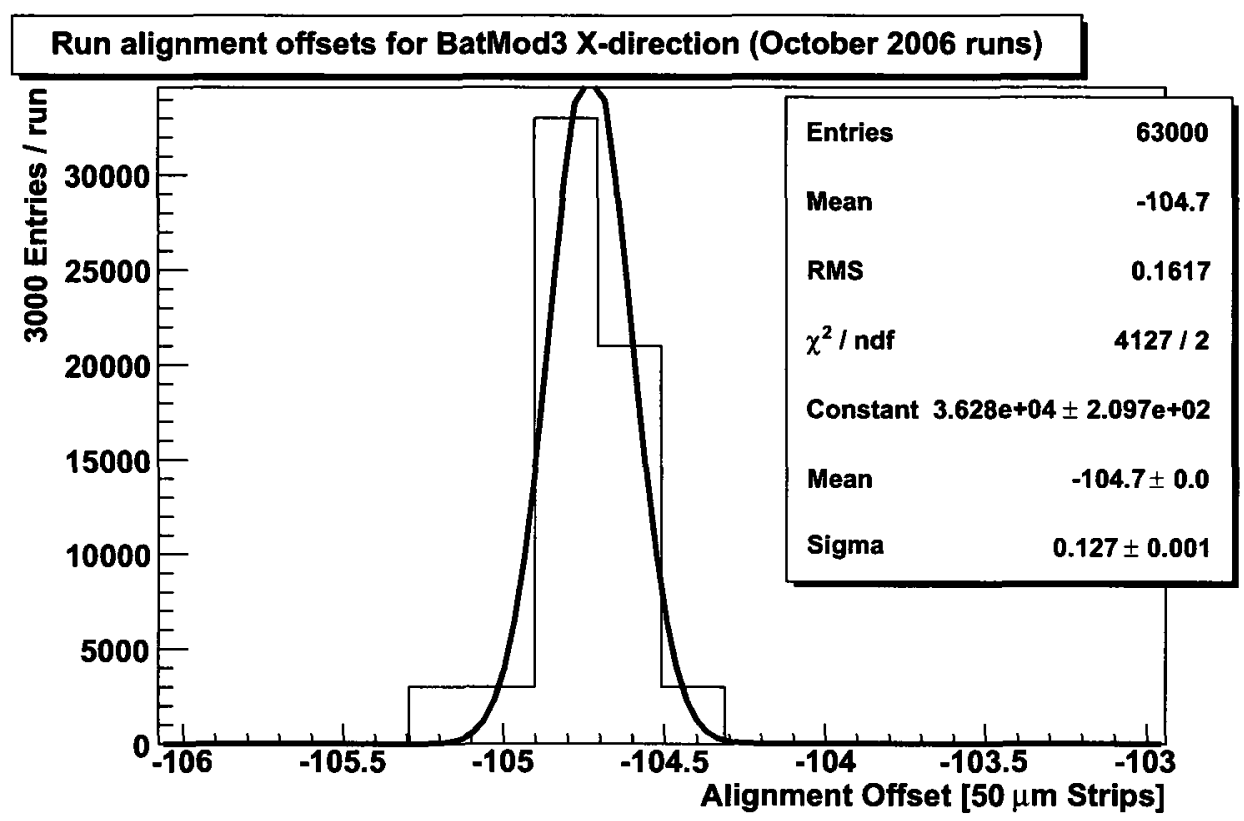

Figure 5.5: Run alignment offsets for BatMod3 in the telescope's X-direction in the October 2006 dataset. The offset peaks at $\mu=-104.7$ strips, corresponding in a $5 \mathrm{~mm}$ offset between the origins of BatMods 3 and 6 in the X-direction of the telescope co-ordinate system. The offset found varies with a width of $\sigma=0.1$ strips across 21 runs, suggesting that the offset is statistically significant from 0 . The Y-axis is scaled by 800 due to the behaviour of the software, but the shape of the distribution is unaffected. 
for pions with $p>100 \mathrm{GeV}$ [18], where $\sigma_{\imath}$ and $z_{\imath}$ are the respective plane resolutions and locations along the beam axis. In the case of the October 2006 data, the DUT is located on the origin of the telescope co-ordinate system. This minimizes the uncertainty on the track. If the plane resolution is taken to be digital, a conservative estimate of the track prediction uncertainty $\sigma_{t} \approx 8 \mu \mathrm{m}$ on the DUT.

The single and double hit residuals for BatMod1 are seen in figures 5.6 (a) and (b) for the October 2006 data. The widths of the distributions shown in Figure 5.6 are indicative of the plane resolution less uncertainty on track reconstruction. The width of the residual distribution was found to be $\sigma=12 \mu \mathrm{m}$ for single hit events, and $\sigma=6 \mu \mathrm{m}$ for double hit events.

\subsection{Charge deposition}

In scCVD diamond the electric field is constant across the full thickness of the sensor as it is not a junction semiconductor. Drift velocity for charge carriers is directly proportional to the applied bias voltage. Higher bias voltages result in lower charge collection times and reduces the spread of the charge cloud caused by diffusion.

How to obtain the most probable charge deposited in an ideal CVD diamond sensor was discussed in Chapter 2. The most probable charge collected in the October 2006 dataset is stable across all runs as seen in Figure 5.7 (b), and yields $\Delta_{p}=(37 \pm 4) \mathrm{BC}$ $=(12000 \pm 1000) \mathrm{e}$. The predicted value of $13400 \mathrm{e}$ for $395 \mu \mathrm{m}$ falls within the $10 \%$ ToT calibration uncertainty, and provides evidence that the detector is functioning as expected. The found value was also in agreement with previous findings from (MATHES). The collected charge distribution can be seen in Figure 5.7 (a), and the most probable collected charge corresponds to a charge collection distance of approximately $410 \mu \mathrm{m}$.

Next we consider the August 2007 dataset, which had CD181 irradiated to $8.5 \times$ 


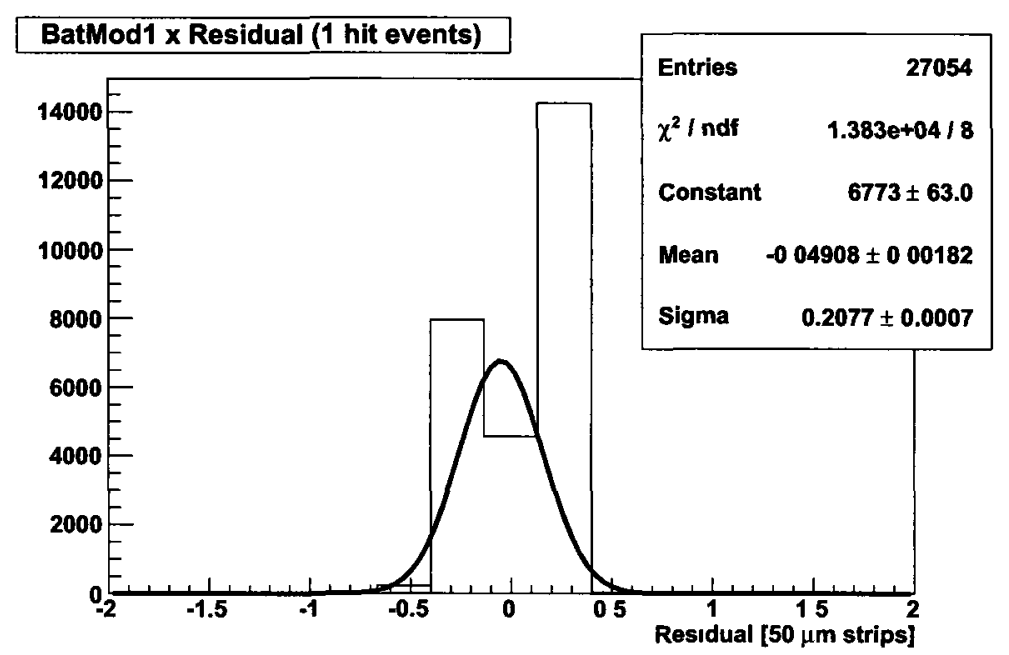

(a) Residual between BatMod1 and fitted track for clusters of 1 hit

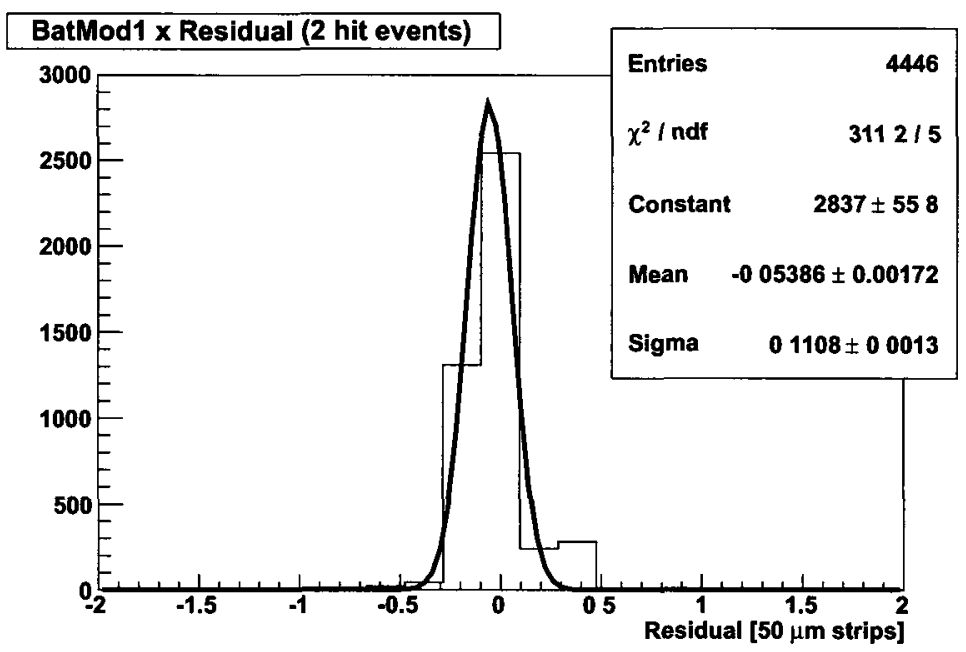

(b) Residual between BatMod1 and fitted track for clusters of 2 hits

Figure 5.6: Residuals between reconstructed hit locations in BatMod1 and fitted tracks. The spatial resolution of the device can be seen in the width of the fitted gaussian. Single hit clusters were found to have a spatial resolution of $12 \mu \mathrm{m}$, and double hit clusters have a spatial resolution of $6 \mu \mathrm{m}$. Because the residual is indicative of the convolution between the track prediction error, multiple scattering and the intrinsic resolution of the BatMods, a clear improvement in telescope resolution can be seen when multiple hits are used to reconstruct the hit location. 


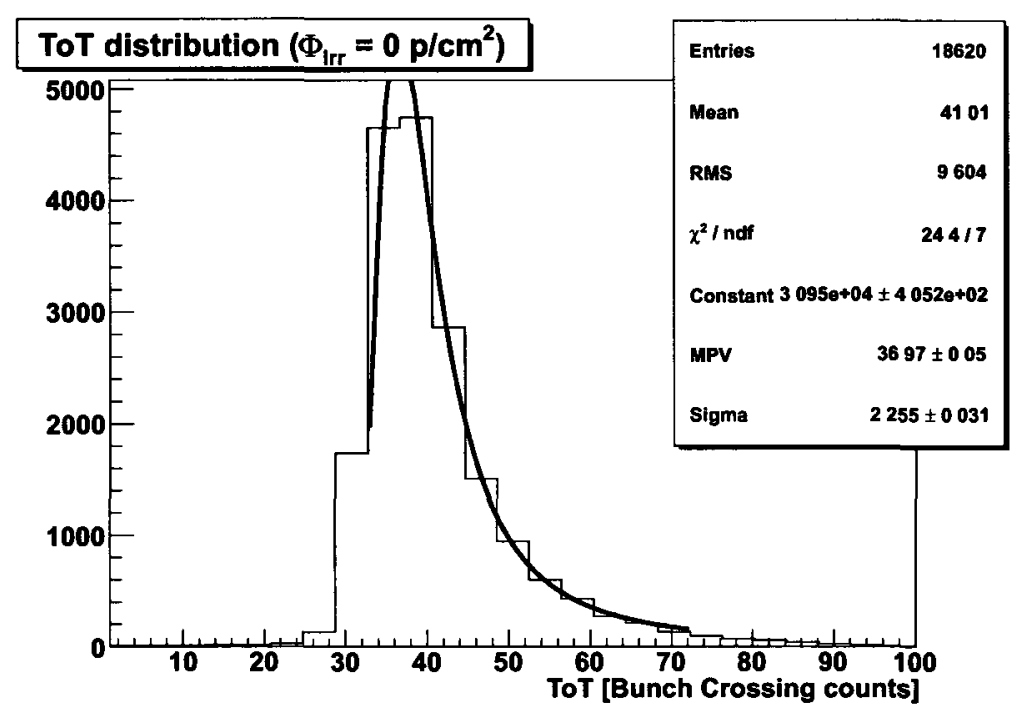

(a) ToT distribution for $\Phi_{\mathrm{Irr}}=0$ protons $/ \mathrm{cm}^{2}$. Distribution corresponds to a mean collected charge of approximately $14800 \mathrm{e}$, or a charge collection distance of $410 \mu \mathrm{m}$, larger than the thickness of the detector

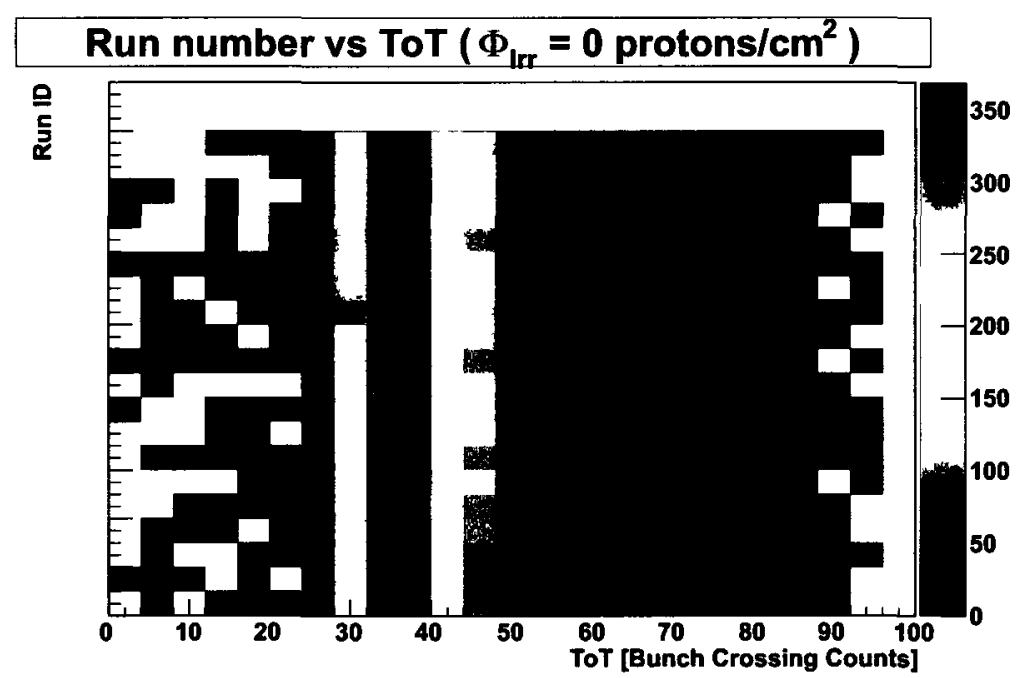

(b) Evolution of ToT distribution over the runs Plot shows the charge distribution of this dataset is stable across all runs.

Figure 5.7: ToT distribution for October 2006 CD181 dataset. Figure (a) shows the summary ToT distribution for the entire dataset. Figure (b) shows the ToT distribution for every considered run. Charge collection properties can be derived from (a), while no deviations in the MPV on a run by run basis are visible in (b). 30 $\mathrm{BC}$ corresponds to 10 ke deposited charge. 
$10^{13}$ protons $/ \mathrm{cm}^{2}$. The evolution of the ToT distribution over the dataset can be seen in Figure 5.8 (b). Like the previous dataset the ToT distribution's MPV is relatively stable across the entirety of the runs. The ToT distribution across the runs is shown in Figure 5.8 (a), and the most probable charge deposition was found to be $\Delta_{p}=32.6 \pm 3=11 \pm 1 \mathrm{ke}$. The noise between $10-20 \mathrm{BC}$ is indicative of early device failure; specifically the degradation of the read-out electronics.

In the July 2008 dataset it becomes clear that device failure is beginning to occur. The evolution of the ToT distribution shown in Figure 5.9 (b), specifically in the later runs where the MPV begins to drop despite regular sensor pumping, and the Landau distribution begins to broaden suggesting that the read-out electronics were beginning to fail. These issues bring the validity of the dataset into question.

The results from the previous test-beam suggested that the radiation tolerance of CD181's read-out electronics had been reached, and the front-end were replaced by a new FE-I3 chipset prior to the June 2009 test-beam. The purpose of the June 2009 test-beam was to perform an angle scan, in an attempt to characterize sensor performance at angles of incidence between $0^{\circ}$ and $15^{\circ}$.

Initial results of the test were promising, with the most probable charge collected value near $30 \mathrm{BC}$; after extended running however, a shift in the MPV was measured, and charge collection began to degrade as it had in the previous run. The sensor was physically manipulated at least nine times during the test, four times for pumping and five for angle adjustments. Initial manipulations for pumping seem to have played a role in temporarily returning functionality to the sensor, but the gradual decline to the point where manipulations no longer affect the charge collected, suggest that hardware failure had occurred. As a result, this test-beam period was removed from consideration in the charge collection analysis.

Once the MPVs were extracted from Figures 5.7, 5.8 and 5.9 (a), the deposited 


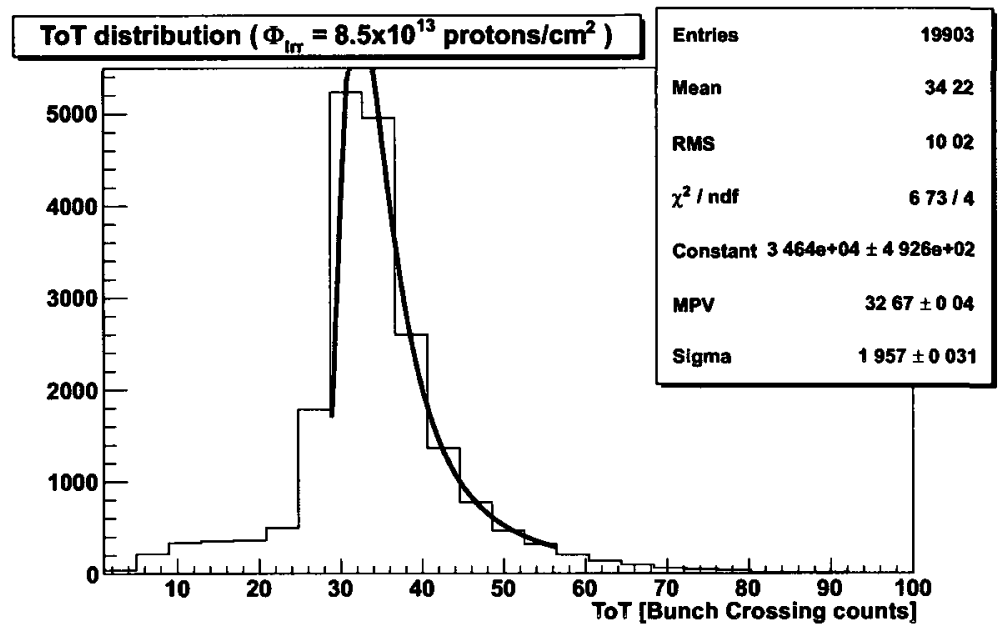

(a) ToT distrıbution for $\Phi_{\text {Irr }}=8.5 \times 10^{13}$ protons $/ \mathrm{cm}^{2}$ A noise peak is forming between 10 and $20 \mathrm{BC}$, consistent with the degradation of the read-out electronics

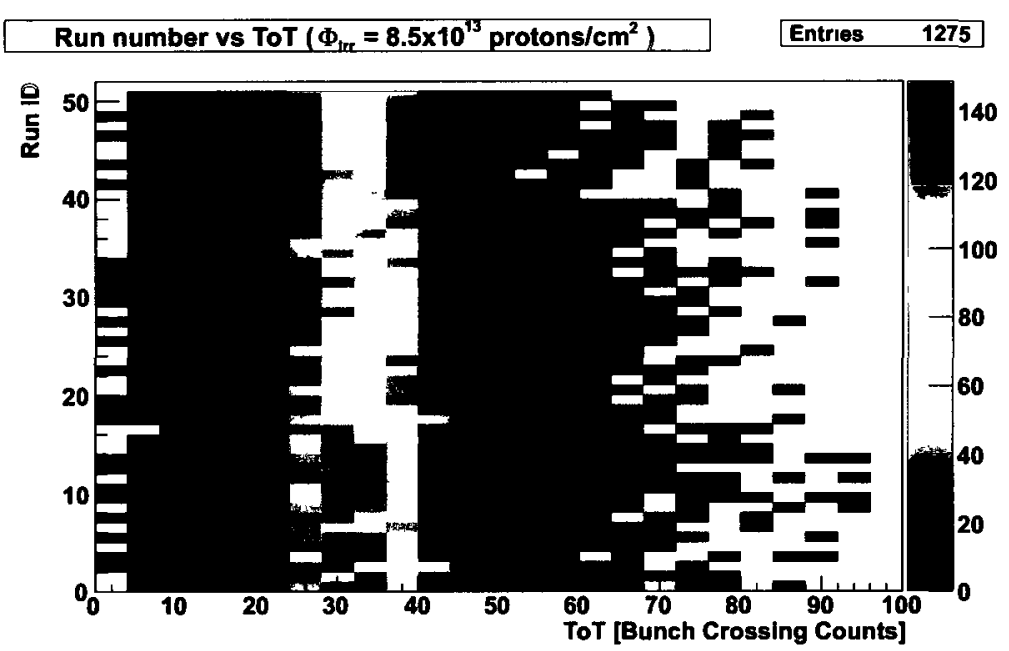

(b) Evolution of ToT distribution over the August 2007 CD181 runs. The ToT is relatively stable across the entirety of the analyzed runs.

Figure 5.8: ToT distribution for August 2007 CD181 dataset. Figure (a) shows the summary ToT distribution for the entire dataset. Figure (b) shows the ToT distribution for every considered run. Charge collection properties can be derived from (a), while no deviations in the MPV on a run by run basis are visible in (b). 30 $\mathrm{BC}$ corresponds to 10 ke deposited charge. 


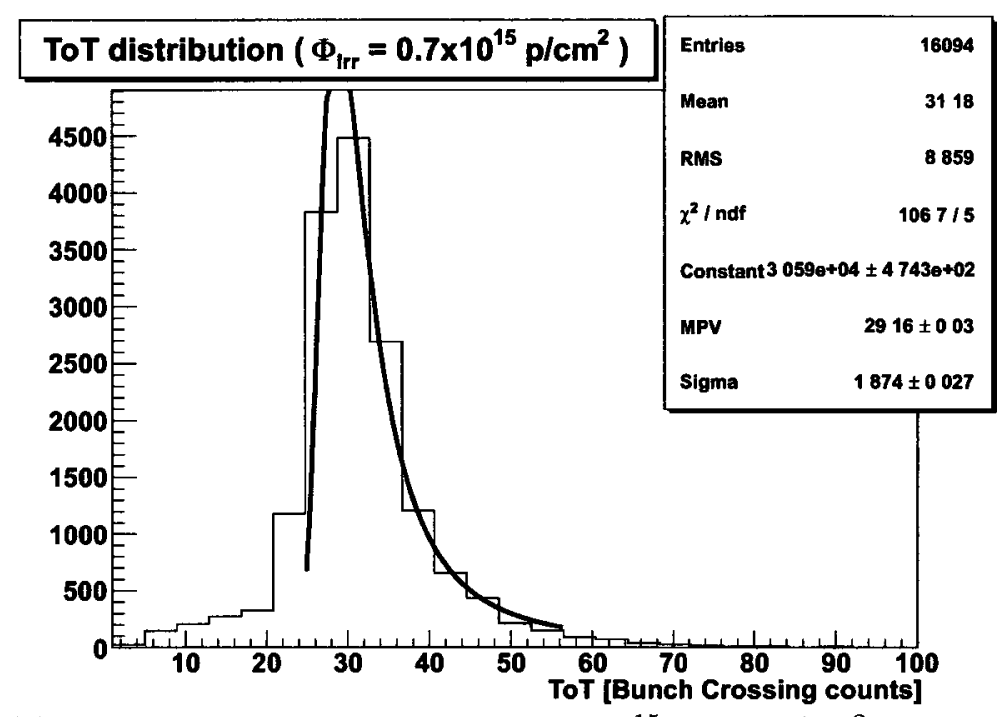

(a) ToT distribution for $\Phi_{\operatorname{Irr}}=07 \times 10^{15}$ protons $/ \mathrm{cm}^{2}$, shows a distortion from the expected Landau charge distribution, especially at low charges. The mean collected charge is approximately $9600 \mathrm{e}$, corresponding to a charge collection distance of $320 \mu \mathrm{m}$.

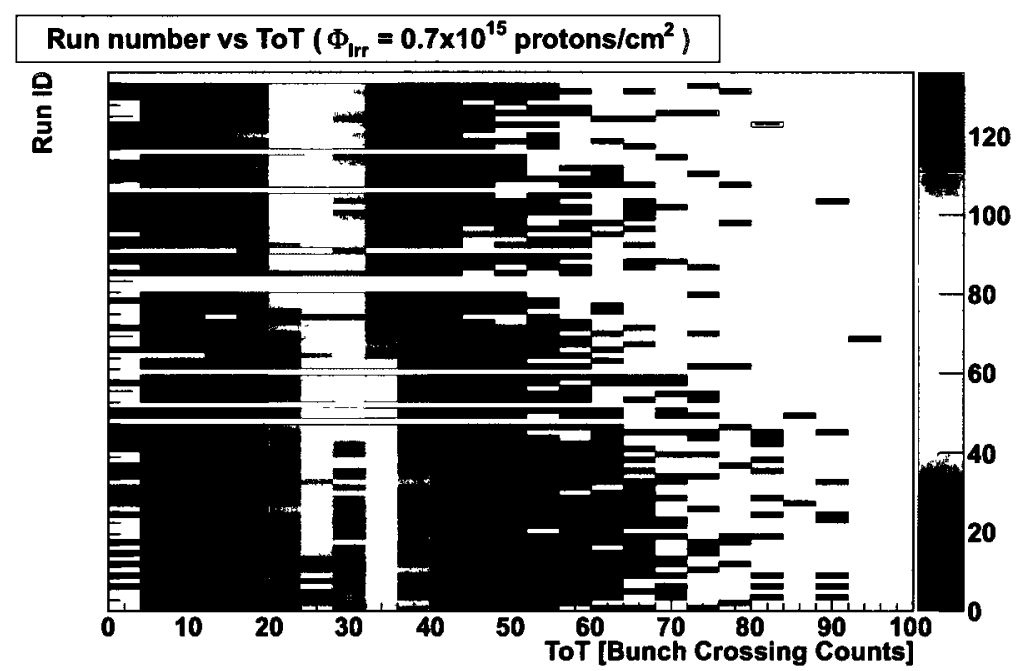

(b) Evolution of ToT distribution over the runs. Plot shows the charge distribution of this dataset is relatively stable in the initial runs, but deviates to a lower MPV approximately mid way through the runs. To combat this effect, only the first 50 runs were considered in the fit.

Figure 5.9: ToT distribution for the July 2008 CD181 dataset. Figure (a) shows the summary ToT distribution for the first 50 runs in dataset. Figure (b) shows the ToT distribution for every run. Charge collection properties can be derived from (a), while deviations in the MPV in later runs basis are clearly visible in (b). $30 \mathrm{BC}$ corresponds to 10ke deposited charge. 


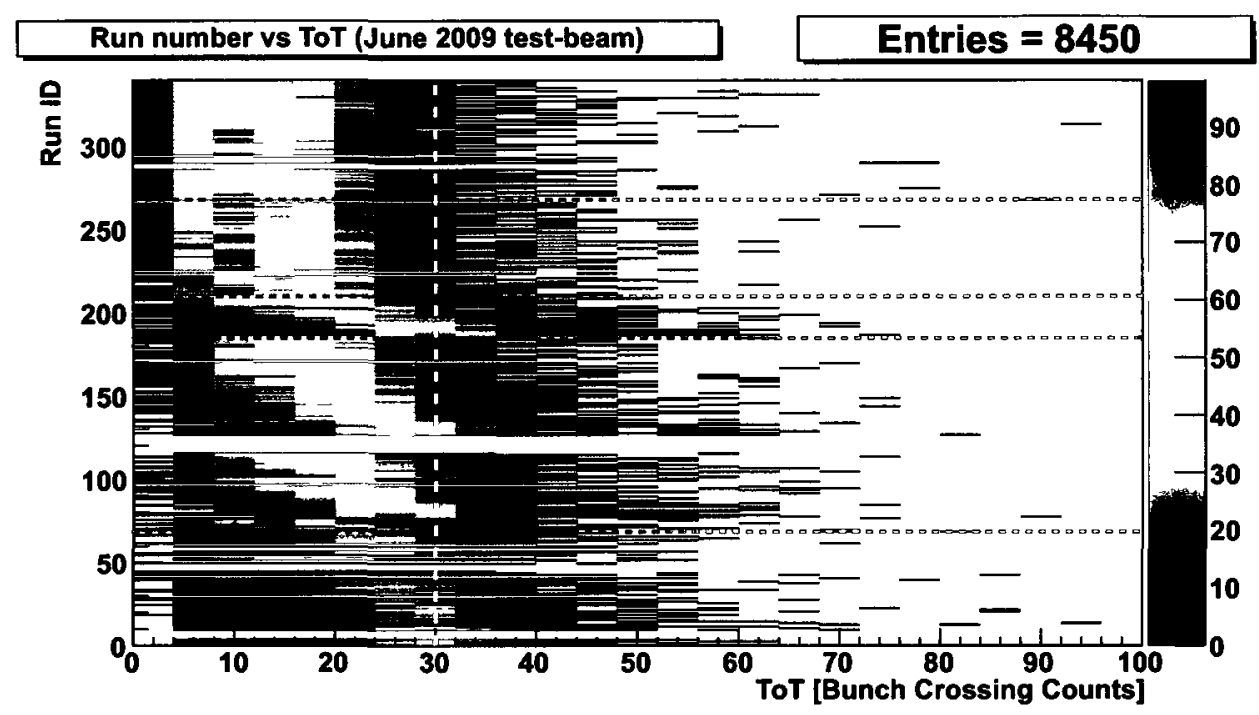

Figure 5.10: Evolution of ToT distribution over the June 2009 CD181 test-beam dataset. Early runs suggest the sensor is functioning properly, but quickly begins shifting towards lower ToT values. The yellow dashed line is a reference $30 \mathrm{BC}$, and the red dashed lines represent points where the sensor was removed from the beam, and pumped for several hours.

charge was determined using equation 5.1, and a charge collection distance was calculated for each dataset. The charge collection distance is given by

$$
\operatorname{ccd}(\Phi)=\frac{Q}{\langle d q / d x\rangle}
$$

where $Q$ is the mean charge collected in the sensor, and $\langle d q / d x\rangle=36 \mathrm{e} / \mu \mathrm{m}$ is the mean charge recombination rate, or the amount of charge which recombines in the sensor per $\mu \mathrm{m}$ of CVD diamond. The damage of CD181 was modelled by characterizing the decay in charge collection distance using a standard damage equation given as

$$
\frac{1}{\operatorname{ccd}\left(\Phi_{\mathrm{Irr}}\right)}=\frac{1}{\operatorname{ccd}(0)}+k_{r} \Phi_{\mathrm{Irr}}
$$

where $1 / c c d(0)$ is the charge collection distance of the sensor prior to irradiation, and $k_{r}$ is the damage constant of the sensor. The damage constant for pCVD diamond 


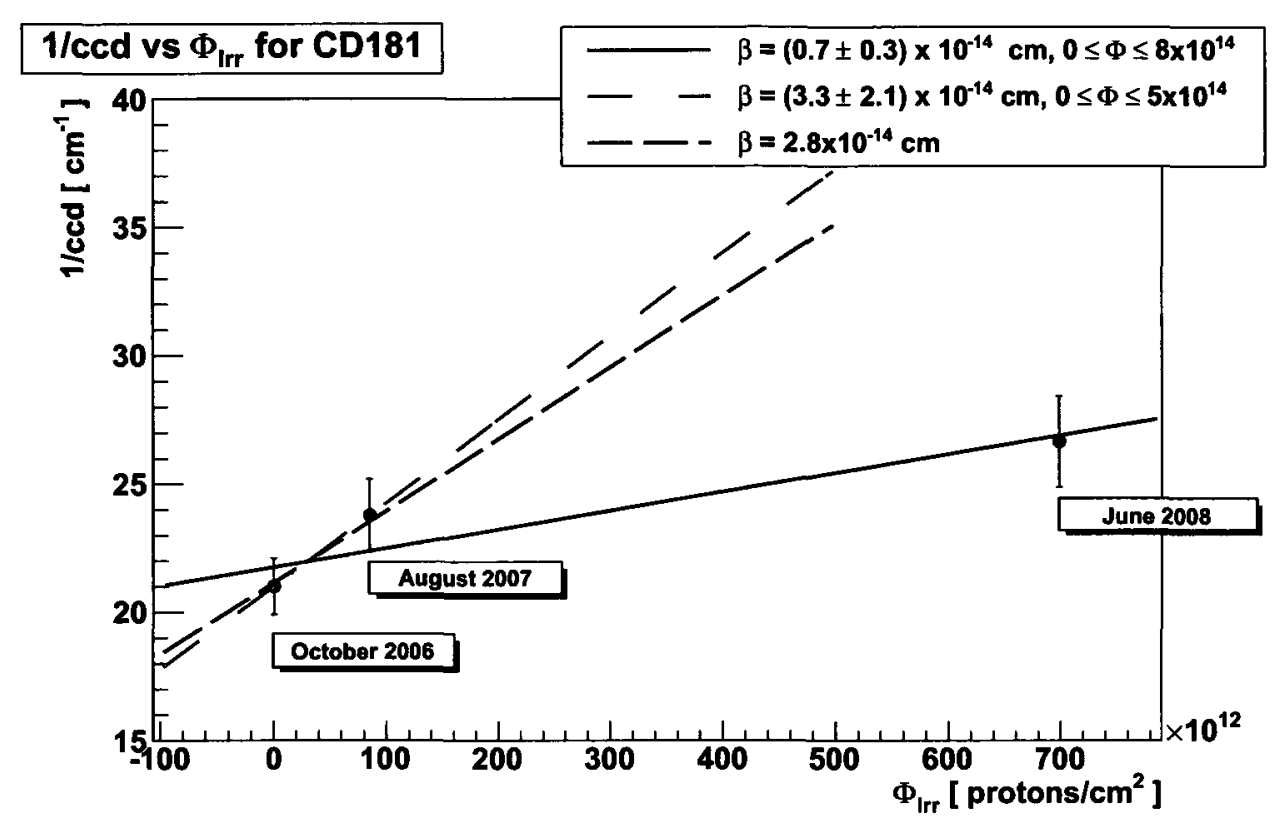

Figure 5.11: Inverse charge collection distance vs irradiation in CD181. Two fits were performed: One including the July 2008 data (solid blue line), and one excluding it (dashed and dotted green line). The fit including the July 2008 dataset found a damage constant of $(0.7 \pm 0.3) \times 10^{-14} \mathrm{~cm}$, the fit excluding the July 2008 dataset found a damage constant of $\left(3.3 \pm 2.1^{-14}\right) \mathrm{cm}$. The fit shown in red fixes the damage rate to the known value at $\beta=2.8 \times 10^{-14} \mathrm{~cm}[30]$ for comparison.

was previously determined to be $(2.8 \pm 0.4) \times 10^{-14} \mathrm{~cm}$ for $24 \mathrm{GeV}$ proton irradiation and $(2.0 \pm 0.5) \times 10^{-14} \mathrm{~cm}$ for $1 \mathrm{MeV}$ neutron irradiation by [30]. To determine the damage constant $k_{r}$, the inverse charge collection distances were calculated in $\mathrm{cm}^{-1}$ and plotted against sensor irradiation in Figure 5.11.

The damage rate for CD181 was found to be $k_{r}=(0.7 \pm 0.3) \times 10^{-14} \mathrm{~cm}$ including the July 2009 dataset in the fit, and $k_{r}=(3.3 \pm 2.1) \times 10^{-14} \mathrm{~cm}$ excluding it. Because the reliability of the June 2008 dataset has been called into question, it would be premature to draw conclusions about the radiation tolerance of CVD diamond with that dataset until its viability has been fully investigated, but the result is $5 \sigma$ consistent with previous findings. The October 2006 and August 2007 data are in full agreement with the findings of [30], and provide evidence that scCVD and pCVD diamond have the same damage constant as expected. 
To summarize, the software developed to analyze data taken with the BonnATLAS telescope is capable of performing several tasks: a simple charge collection analysis has been run, and has determined a damage constant found to be consistent with previous results. Rudimentary telescope plane alignments may be generated which are stable across entire datasets to within 0.1 strips of a reference plane. Track reconstruction is possible, but extrapolation into the DUT has yet to be proven to work. The software also contains several dataset diagnostic tools which display ToT distributions in a user friendly manner.

In order to be complete, the code would have to incorporate DUTs and consider rotations during device alignment. Tracking information obtained from the code could be used to determine the spatial resolution, and hit reconstruction efficiency of DUTs. Special care could also be taken to correct event ID miscounts to increase the statistics of old datasets. 


\section{Chapter 6}

\section{Summary}

Future colliders in experimental particle physics such as the sLHC will require detectors close to the interaction point to endure radiation levels a full order of magnitude beyond their current rated abilities. Research is ongoing to develop detectors capable of withstanding the levels of radiation present in the sLHC's tracker environment. The three most promising sensor concepts are planar and 3D silicon, which use new junction structures to improve radiation tolerance, and diamond which replaces the sensing material entirely.

The theory underlying semiconducting particle trackers, and the requirements for radiation tolerant detectors were outlined in chapter 2. An accurate prediction of charge collection properties in diamond were derived for the transition of high energy particles in a diamond sensor, and the properties of electrical grade CVD diamonds including their synthesis were described. The ATLAS FE-I3 pixel read-out electronics and the specifics of their operation were outlined in chapter 3 .

I performed the installation and testing of the tools necessary to characterize sensors using ATLAS pixel read-out electronics at Carleton university, and the process was documented in chapter 4 . The process for sensor calibration was demonstrated. It was found that the USBPix data-acquisition and calibration software were capable

of calibrating the provided sensor planar silicon chip 10-7A to standard operational 
parameters. The threshold of the chip was tuned to $(4000 \pm 20)$ e, with an operational noise of $(162 \pm 7) \mathrm{e}$, and the noise measured in each pixel was used to create a pixel mask, increasing the efficiency of the detector. A ToT calibration was obtained, and the system was capable of reconstructing $20 \mathrm{ke}$ of injected charge with ToT count of $(29.9 \pm 0.4)$ bunch crossings, well within the inputted parameters of the calibration.

I performed a source scan using a Sr-90 beta source was performed, and a most probable charge collected across all cluster sizes of $\Delta_{p}=(14 \pm 1)$ ke was found, and it is estimated that the calibration is underestimating charge deposition by approximately $29 \%$. The setup shows promise for use in tracking applications, and may be used as a beam telescope for studies in other detector upgrade beam tests.

The tools I developed were outlined in chapter 5 with the express purpose of analyzing the data obtained for a single crystal CVD diamond, CD181, at increasing particle fluences using the Bonn-ATLAS telescope. It was demonstrated that the analysis suite was capable of rudimentary device alignment to within a strip of charged particle tracks in the BAT discounting plane rotations.

Bonn-ATLAS telescope modules were found to have an intrinsic resolution of $\sigma \approx 8 \mu \mathrm{m}$. Module alignment was demonstrated to vary by $5 \mu \mathrm{m}$ across 21 runs, implying the algorithm chosen is capable of producing stable alignments. Reconstructed tracks were found to have prediction errors on the order of $5 \mu \mathrm{m}$ in the device under test. Alignment and track reconstruction techniques from previous analysis software produced resolutions comparable to those found with the new software.

I performed a preliminary analysis of the datasets taken between October 2006 and June 2009 to determine if the data met quality standards using tools I developed. It was determined that the October 2006 and August 2007 datasets were stable and met data quality standards. It was found that hardware complications contributed to insurmountable degradation in the quality of the July 2008 and June 2009 datasets, 
and they were removed from consideration in the analysis. These tools are being modified by the existing diamond pixel group to expand their capabilities, and perform a more complete analysis.

The observed most probable charge deposition of $\Delta_{p}=(12 \pm 1)$ ke for CD181 in October 2006 and the measured damage constant $\beta_{e}=3 \pm 2 \mathrm{~cm}$ for the two considered datasets were found to be in agreement with previous findings. The current size of scCVD diamonds is limited to $1 \mathrm{~cm}^{2}$, and they are not produced in large enough quantities to produce full ATLAS pixel modules. Several facets of sensor performance beyond charge collection remain to be characterized before a diamond sensor will be introduced into the ATLAS pixel detector.

Large area pCVD diamonds can be produced to the sizes required for full ATLAS pixel modules, but sufficient data has yet to be obtained for an irradiated sensor to determine their suitability for use in the ATLAS detector. Sensor tests have been performed and are being planned to characterize pCVD sensors irradiated with sLHC fluences under operational parameters.

To summarize, I have demonstrated that I have produced a test-stand at Carleton capable of calibrating, characterizing and controlling ATLAS FE-I3 chips using the USBPix system. It is performing as intended, and is currently being adapted to be used as a rudimentary particle telescope. I have also demonstrated using the software I developed that the charge collection of scCVD diamond devices have a charge collection distance damage constant on the order of (or potentially better than) silicon up to $8.5 \times 10^{13}$ protons $/ \mathrm{cm}^{2}$. While this falls short of the radiation expected in the sLHC, device failures beyond my control and circumstance prevented a reliable study of the sensor to that level of irradiation. 


\section{References}

[1] P. W. Higgs, Phys. Rev. Lett. 13, 508 (1964).

[2] ATLAS Collaboration, ATLAS public webpage, http://www.atlas.ch/, 2010, [Online].

[3] G. Aad et al., JINST 3, S08003 (2008).

[4] G. Azuelos et al., Journal of Physics G: Nuclear and Particle Physics 28, 2453 (2002).

[5] ATLAS Collaboration upgrade group, ATLAS upgrade group webpage, http: //atlas.web.cern.ch/Atlas/GROUPS/UPGRADES/, 2010, [Online].

[6] M. Karagounis, (2008).

[7] R. Collaboration, CERN RD50 Collaboration home page, http://rd50.web. cern.ch/rd50/, 2010, [Online].

[8] R. Collaboration, CERN RD39 Collaboration home page , http://ww.hip.fi/ research/cms/tracker/RD39/php/home.php, 2010, [Online].

[9] R. Collaboration, CERN RD42 Collaboration home page, http://greybook. cern.ch/programmes/experiments/RD42.html, 2010, [Online].

[10] K. Nakamura et al., Journal of Physics G 37, 075021 (2010).

[11] J. Lindhard et al., Kgl. Danske Videnskab. Selskab, Mat.-Fys. Medd. 33, (1963).

[12] H. Andersen and J. Ziegler, Hydrogen: Stopping Powers and Ranges in All Elements. Vol. 3 of The Stopping and Ranges of Ions in Matter (Pergamon Press, New York, 1977).

[13] H. Bichsel, Rev. Mod. Phys. 60, 663 (1988).

[14] L.D. Landau, J. Exp. Phys. (USSR) 8:201+ (1944). 
[15] L. Rossi, P. Fischer, T. Rohe, and N. Wermes, Prxel Detectors: From Fundamentals to Applications (Particle Acceleration and Detection) (Springer, 11 West 42nd Street, 15th Floor, New York, NY 10036, 2006).

[16] M. Keil et al., Nuclear Instruments and Methods in Physics Research Section A: Accelerators, Spectrometers, Detectors and Associated Equipment 501, 153 (2003), proceedings of the 10th International Workshop on Vertex Detectors.

[17] Element Six Technologies, E6 CVD diamond eShop, http://www.e6cvd.com/, 2010, [Online; accessed 16-Sept-2010].

[18] M. Mathes, Ph.D. thesis, Bonn University, 2008.

[19] R. S. Wallny, Nuclear Instruments and Methods in Physics Research Section A: Accelerators, Spectrometers, Detectors and Associated Equipment 582, 824 (2007), vERTEX 2006 - Proceedings of the 15th International Workshop on Vertex Detectors.

[20] ATLAS-Canada Collaboration, ATLAS-Canada public page, http://www. atlas-canada.ca/, 2010, [Online].

[21] The Rose Collaboration, Notes on the fluence normalization based on the NIEL scaling hypothesis., http://rd48.web.cern.ch/, 2000, technical note: ROSE/TN/2000-02.

[22] G. Lindstrm et al., Nuclear Instruments and Methods in Physics Research Section A: Accelerators, Spectrometers, Detectors and Associated Equipment 466, 308 (2001).

[23] A. Vasilescu and, G. Lindstroem, Displacement Damage in Silicon, http:// sesam.desy.de/members/gunnar/Si-dfuncs.html, 2000, [Online compilation].

[24] M. Moll, Ph.D. thesis, Hamburg University, 1999.

[25] M. Moll and B. Mara, Technical Report No. LHCC-RD-015. CERN-LHCC-2008001, CERN, Geneva (unpublished).

[26] M. Bruinsma et al., Nucl. Phys. Proc. Suppl. 150, 164 (2006).

[27] H. Kagan, S. Roe, and P. Weilhammer, Technical Report No. LHCC-RD-016. CERN-LHCC-2008-005, CERN, Geneva (unpublished).

[28] D. Asner and others, Nucl. Instr. and Meth. A (2010). 
[29] I. Peric et al., Nuclear Instruments and Methods in Physics Research Section A: Accelerators, Spectrometers, Detectors and Associated Equipment 565, 178 (2006), proceedings of the International Workshop on Semiconductor Pixel Detectors for Particles and Imaging - PIXEL 2005.

[30] W. Adam et al., Nuclear Instruments and Methods in Physics Research Section A: Accelerators, Spectrometers, Detectors and Associated Equipment 447, 244 (2000).

[31] Z. Li et al., Nuclear Instruments and Methods in Physics Research Section A: Accelerators, Spectrometers, Detectors and Associated Equipment 461, 126 (2001), 8th Pisa Meeting on Advanced Detectors.

[32] Z. Li et al., Nuclear Science, IEEE Transactions on 39, 1730 (1992).

[33] J.J. Velthuis, Ph.D. thesis, NIKHEF, Amsterdam, The Netherlands, 2003.

[34] H. Krueger et al., USBPix - USB based readout system for ATLAS FE-I3 and FEI4, http://icwiki.physik. uni-bonn.de/twiki/bin/view/Systems/UsbPix, 2000, [Online wiki].

[35] J. Treis et al., Nuclear Instruments and Methods in Physics Research Section A: Accelerators, Spectrometers, Detectors and Associated Equipment 490, 112 (2002). 